\author{
R. Phillip Dellinger \\ Mitchell M. Levy \\ Jean M. Carlet \\ Julian Bion \\ Margaret M. Parker \\ Roman Jaeschke \\ Konrad Reinhart \\ Derek C. Angus \\ Christian Brun-Buisson \\ Richard Beale \\ Thierry Calandra \\ Jean-Francois Dhainaut \\ Herwig Gerlach \\ Maurene Harvey \\ John J. Marini \\ John Marshall \\ Marco Ranieri \\ Graham Ramsay \\ Jonathan Sevransky \\ B. Taylor Thompson \\ Sean Townsend \\ Jeffrey S. Vender \\ Janice L. Zimmerman \\ Jean-Louis Vincent
}

Received: 3 August 2007

Accepted: 25 October 2007

Published online: 4 December 2007

(C) Society of Critical Care Medicine 2007

The article will also be published in Critical Care Medicine.

* Sponsor of 2004 guidelines; ** Sponsor of 2008 guidelines but did not participate formally in revision process; $* * *$ Members of the 2007 SSC Guidelines Committee are listed in Appendix I.; **** Please see Appendix $\mathrm{J}$ for author disclosure information.

for the International Surviving Sepsis Campaign Guidelines Committee ${ }^{* * *}$,****

Sponsoring Organizations: American Association of Critical-Care Nurses*, American College of Chest Physicians*, American College of Emergency Physicians*, Canadian Critical Care Society, European Society of Clinical Microbiology and Infectious Diseases*, European Society of Intensive Care Medicine*, European Respiratory Society*, International Sepsis Forum*, Japanese Association for Acute Medicine, Japanese Society of Intensive Care Medicine, Society of Critical Care Medicine*, Society of Hospital Medicine**, Surgical Infection Society*, World Federation of Societies of Intensive and Critical Care

\title{
Surviving Sepsis Campaign: International guidelines for management of severe sepsis and septic shock: 2008
}

Medicine**. Participation and endorsement by the German Sepsis Society and the Latin American Sepsis Institute.

\section{R. P. Dellinger $(\square)$}

Cooper University Hospital,

One Cooper Plaza, 393 Dorrance,

Camden 08103, NJ, USA

e-mail: Dellinger-Phil@CooperHealth.edu

M. M. Levy $\cdot$ S. Townsend

Rhode Island Hospital,

Providence RI, USA

J. M. Carlet

Hospital Saint-Joseph,

Paris, France

J. Bion

Birmingham University,

Birmingham, UK

M. M. Parker

SUNY at Stony Brook,

Stony Brook NY, USA

R. Jaeschke

McMaster University,

Hamilton, Ontario, Canada

K. Reinhart

Friedrich-Schiller-University of Jena, Jena, Germany
D. C. Angus

University of Pittsburgh,

Pittsburgh PA, USA

C. Brun-Buisson

Hopital Henri Mondor,

Créteil, France

R. Beale

Guy's and St Thomas' Hospital Trust, London, UK

T. Calandra

Centre Hospitalier Universitaire Vaudois, Lausanne, Switzerland

\section{J.-F. Dhainaut}

French Agency for Evaluation of Research and Higher Education,

Paris, France

H. Gerlach

Vivantes-Klinikum Neukoelln,

Berlin, Germany

\section{Harvey}

Consultants in Critical Care, Inc.,

Glenbrook NV, USA

J. J. Marini

University of Minnesota,

St. Paul MN, USA 
J. Marshall

St. Michael's Hospital,

Toronto, Ontario, Canada

M. Ranieri

Università di Torino,

Torino, Italy

G. Ramsay

West Hertfordshire Health Trust,

Hemel Hempstead, UK

J. Sevransky

The Johns Hopkins University School

of Medicine,

Baltimore MD, USA

B. T. Thompson

Massachusetts General Hospital,

Boston MA, USA

J.S. Vender

Evanston Northwestern Healthcare,

Evanston IL, USA

\section{J. L. Zimmerman}

The Methodist Hospital,

Houston TX, USA

\section{J.-L. Vincent}

Erasme University Hospital,

Brussels, Belgium

Abstract Objective: To provide an update to the original Surviving Sepsis Campaign clinical management guidelines, "Surviving Sepsis Campaign guidelines for management of severe sepsis and septic shock," published in 2004. Design: Modified Delphi method with a consensus conference of 55 international experts, several subsequent meetings of subgroups and key individuals, teleconferences, and electronic-based discussion among subgroups and among the entire committee. This process was conducted independently of any industry funding. Methods: We used the GRADE system to guide assessment of quality of evidence from high (A) to very low (D) and to determine the strength of recommendations. A strong recommendation [1] indicates that an intervention's desirable effects clearly outweigh its undesirable effects (risk, burden, cost), or clearly do not. Weak recommendations [2] indicate that the tradeoff between desirable and undesirable effects is less clear. The grade of strong or weak is considered of greater clinical importance than a difference in letter level of quality of evidence. In areas without complete agreement, a formal process of resolution was developed and applied. Recommendations are grouped into those directly targeting severe sepsis, recommendations targeting general care of the critically ill patient that are considered high priority in severe sepsis, and pediatric considerations. Results: Key recommendations, listed by category, include: early goal-directed resuscitation of the septic patient during the first $6 \mathrm{hrs}$ after recognition (1C); blood cultures prior to antibiotic therapy (1C); imaging studies performed promptly to confirm potential source of infection (1C); administration of broadspectrum antibiotic therapy within $1 \mathrm{hr}$ of diagnosis of septic shock (1B) and severe sepsis without septic shock (1D); reassessment of antibiotic therapy with microbiology and clinical data to narrow coverage, when appropriate (1C); a usual 7-10 days of antibiotic therapy guided by clinical response (1D); source control with attention to the balance of risks and benefits of the chosen method (1C); administration of either crystalloid or colloid fluid resuscitation (1B); fluid challenge to restore mean circulating filling pressure (1C); reduction in rate of fluid administration with rising filing pressures and no improvement in tissue perfusion (1D); vasopressor preference for norepinephrine or dopamine to maintain an initial target of mean arterial pressure $\geq 65 \mathrm{~mm} \mathrm{Hg}$ (1C); dobutamine inotropic therapy when cardiac output remains low despite fluid resuscitation and combined inotropic/vasopressor therapy (1C); stress-dose steroid therapy given only in septic shock after blood pressure is identified to be poorly responsive to fluid and vasopressor therapy (2C); recombinant activated protein $\mathrm{C}$ in patients with severe sepsis and clinical assessment of high risk for death (2B except $2 \mathrm{C}$ for postoperative patients). In the absence of tissue hypoperfusion, coronary artery disease, or acute hemorrhage, target a hemoglobin of $7-9 \mathrm{~g} / \mathrm{dL}$ (1B); a low tidal volume (1B) and limitation of inspiratory plateau pressure strategy (1C) for acute lung injury (ALI)/ acute respiratory distress syndrome (ARDS); application of at least a minimal amount of positive endexpiratory pressure in acute lung injury (1C); head of bed elevation in mechanically ventilated patients unless contraindicated (1B); avoiding routine use of pulmonary artery catheters in ALI/ARDS (1A); to decrease days of mechanical ventilation and ICU length of stay, a conservative fluid strategy for patients with established ALI/ARDS who are not in shock (1C); protocols for weaning and sedation/analgesia (1B); using either intermittent bolus sedation or continuous infusion sedation with daily interruptions or lightening (1B); avoidance of neuromuscular blockers, if at all possible (1B); institution of glycemic control (1B) targeting a blood glucose $<150 \mathrm{mg} / \mathrm{dL}$ after initial stabilization ( $2 \mathrm{C}$ ); equivalency of continuous veno-veno hemofiltration or intermittent hemodialysis (2B); prophylaxis for deep vein thrombosis (1A); use of stress ulcer prophylaxis to prevent upper GI bleeding using $\mathrm{H} 2$ blockers (1A) or proton pump inhibitors (1B); and consideration of limitation of support where appropriate (1D). Recommendations specific to pediatric severe sepsis include: greater use of physical examination therapeutic end points (2C); dopamine as the first drug of choice for hypotension (2C); steroids only in children with suspected or proven adrenal insufficiency (2C); a recommendation against the use of recombinant activated protein $\mathrm{C}$ in children (1B). Conclusion: There was strong agreement among a large cohort of international experts regarding many level 1 recommendations for the best current care of patients with severe sepsis. Evidenced-based recommendations regarding the acute management of sepsis and septic shock are the first step toward improved outcomes for this important group of critically ill patients.

Keywords Sepsis - Severe sepsis . Septic shock - Sepsis syndrome · Infection - GRADE - Guidelines . Evidence-based medicine $\cdot$ Surviving Sepsis Campaign - Sepsis bundles 


\section{Introduction}

Severe sepsis (acute organ dysfunction secondary to infection) and septic shock (severe sepsis plus hypotension not reversed with fluid resuscitation) are major healthcare problems, affecting millions of individuals around the world each year, killing one in four (and often more), and increasing in incidence [1-5]. Similar to polytrauma, acute myocardial infarction, or stroke, the speed and appropriateness of therapy administered in the initial hours after severe sepsis develops are likely to influence outcome. In 2004, an international group of experts in the diagnosis and management of infection and sepsis, representing 11 organizations, published the first internationally accepted guidelines that the bedside clinician could use to improve outcomes in severe sepsis and septic shock $[6,7]$. These guidelines represented Phase II of the Surviving Sepsis Campaign (SSC), an international effort to increase awareness and improve outcomes in severe sepsis. Joined by additional organizations, the group met again in 2006 and 2007 to update the guidelines document using a new evidence-based methodology system for assessing quality of evidence and strength of recommendations [8-11].

These recommendations are intended to provide guidance for the clinician caring for a patient with severe sepsis or septic shock. Recommendations from these guidelines cannot replace the clinician's decision-making capability when he or she is provided with a patient's unique set of clinical variables. Most of these recommendations are appropriate for the severe sepsis patient in both the intensive care unit (ICU) and non-ICU settings. In fact the committee believes that, currently, the greatest outcome improvement can be made through education and process change for those caring for severe sepsis patients in the non-ICU setting and across the spectrum of acute care. It should also be noted that resource limitations in some institutions and countries may prevent physicians from accomplishing particular recommendations.

\section{Methods}

Sepsis is defined as infection plus systemic manifestations of infection (Table 1) [12]. Severe sepsis is defined as sepsis plus sepsis-induced organ dysfunction or tissue hypoperfusion. The threshold for this dysfunction has varied somewhat from one severe sepsis research study to another. An example of typical thresholds identification of severe sepsis is shown in Table 2 [13]. Sepsis induced hypotension is defined as a systolic blood pressure(SBP) of $<90 \mathrm{~mm} \mathrm{Hg}$ or mean arterial pressure $<70 \mathrm{~mm} \mathrm{Hg}$ or a SBP decrease $>40 \mathrm{~mm} \mathrm{Hg}$ or $<2$ SD below normal for age in the absence of other causes of hypotension. Septic shock is defined as sepsis induced hypotension
Table 1 Determination of the Quality of Evidence

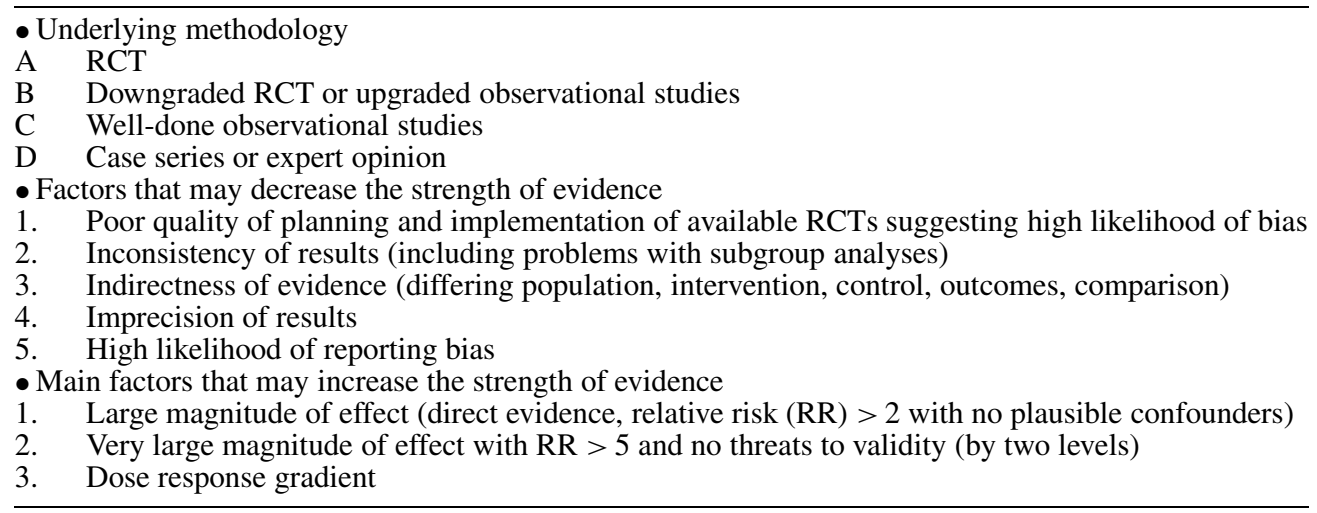

$R C T$, randomized controlled trial; $R R$, relative risk

Table 2 Factors Determining Strong vs. Weak Recommendation

\begin{tabular}{ll}
\hline What should be considered & Recommended Process \\
\hline Quality of evidence & The lower the quality of evidence the less likely a strong recommendation \\
Relative importance of the outcomes & If values and preferences vary widely, a strong recommendation becomes less likely \\
Baseline risks of outcomes & The higher the risk, the greater the magnitude of benefit \\
Magnitude of relative risk including & Larger relative risk reductions or larger increases in relative risk of harm make a strong \\
benefits, harms, and burden & recommendation more or less likely respectively \\
Absolute magnitude of the effect & The larger the absolute benefits and harms, the greater or \\
& lesser likelihood respectively of a strong recommendation \\
Precision of the estimates of the effects & The greater the precision the more likely is a strong recommendation \\
Costs & The higher the cost of treatment, the less likely a strong recommendation \\
\hline
\end{tabular}


persisting despite adequate fluid resuscitation. Sepsis induced tissue hypoperfusion is defined as either septic shock, an elevated lactate or oliguria.

The current clinical practice guidelines build on the first and second editions from 2001 (see below) and 2004 [6, 7, 14]. The 2001 publication incorporated a MEDLINE search for clinical trials in the preceding 10 years, supplemented by a manual search of other relevant journals [14]. The 2004 publication incorporated the evidence available through the end of 2003. The current publication is based on an updated search into 2007 (see methods and rules below).

The 2001 guidelines were coordinated by the International Sepsis Forum (ISF); the 2004 guidelines were funded by unrestricted educational grants from industry and administered through the Society of Critical Care Medicine (SCCM), the European Society of Intensive Care Medicine (ESICM), and ISF. Two of the SSC administering organizations receive unrestricted industry funding to support SSC activities (ESICM and SCCM), but none of this funding was used to support the 2006-2007 committee meetings.

It is important to distinguish between the process of guidelines revision and the Surviving Sepsis Campaign. The Surviving Sepsis Campaign (SSC) is partially funded by unrestricted educational industry grants, including those from Edwards LifeSciences, Eli Lilly and Company, and Philips Medical Systems. SSC also received funding from the Coalition for Critical Care Excellence of the Society of Critical Care Medicine. The great majority of industry funding has come from Eli Lilly and Company.

Current industry funding for the Surviving Sepsis Campaign is directed to the performance improvement initiative. No industry funding was used in the guidelines revision process.

For both the 2004 and the 2006/2007 efforts there were no members of the committee from industry, no industry input into guidelines development, and no industry presence at any of the meetings. Industry awareness or comment on the recommendations was not allowed. No member of the guideline committee received any honoraria for any role in the 2004 or 2006/2007 guidelines process. The committee considered the issue of recusement of individual committee members during deliberation and decision making in areas where committee members had either financial or academic competing interests; however, consensus as to threshold for exclusion could not be reached. Alternatively, the committee agreed to ensure full disclosure and transparency of all committee members' potential conflicts at time of publication (see disclosures at the end of this document).

The guidelines process included a modified Delphi method, a consensus conference, several subsequent meetings of subgroups and key individuals, teleconferences and electronically based discussions among subgroups and members of the entire committee and two follow-up nominal group meetings in 2007.

Subgroups were formed, each charged with updating recommendations in specific areas, including corticosteroids, blood products, activated protein $\mathrm{C}$, renal replacement therapy, antibiotics, source control, and glucose control, etc. Each subgroup was responsible for updating the evidence (into 2007, with major additional elements of information incorporated into the evolving manuscript throughout 2006 and 2007). A separate search was performed for each clearly defined question. The committee chair worked with subgroup heads to identify pertinent search terms that always included, at a minimum, sepsis, severe sepsis, septic shock and sepsis syndrome crossed against the general topic area of the subgroup as well as pertinent key words of the specific question posed. All questions of the previous guidelines publications were searched, as were pertinent new questions generated by general topic related search or recent trials. Quality of evidence was judged by pre-defined Grades of Recommendation, Assessment, Development and Evaluation (GRADE) criteria (see below). Significant education of committee members on the GRADE approach was performed via email prior to the first committee meeting and at the first meeting. Rules were distributed concerning assessing the body of evidence and GRADE experts were available for questions throughout the process. Subgroups agreed electronically on draft proposals that were presented to committee meetings for general discussion. In January 2006, the entire group met during the 35th SCCM Critical Care Congress in San Francisco, California, USA. The results of that discussion were incorporated into the next version of recommendations and again discussed using electronic mail. Recommendations were finalized during nominal group meetings (composed of a subset of the committee members) at the 2007 SCCM (Orlando) and 2007 International Symposium on Intensive Care and Emergency Medicine (Brussels) meetings with recirculation of deliberations and decisions to the entire group for comment or approval. At the discretion of the chair and following adequate discussion, competing proposals for wording of recommendations or assigning strength of evidence were resolved by formal voting. On occasions, voting was performed to give the committee a sense of distribution of opinions to facilitate additional discussion. The manuscript was edited for style and form by the writing committee with final approval by section leads for their respective group assignment and then by the entire committee.

The development of guidelines and grading of recommendations for the 2004 guideline development process were based on a system proposed by Sackett in 1989, during one of the first American College of Chest Physicians (ACCP) conferences on the use of antithrombotic therapies [15]. The revised guidelines recommendations are based on the Grades of Recommendation, Assessment, 
Development and Evaluation (GRADE) system - a structured system for rating quality of evidence and grading strength of recommendation in clinical practice [8-11]. The SSC Steering Committee and individual authors collaborated with GRADE representatives to apply the GRADE system to the SSC guidelines revision process. The members of GRADE group were directly involved, either in person or via e-mail, in all discussions and deliberations amongst the guidelines committee members as to grading decisions. Subsequently, the SSC authors used written material prepared by the GRADE group and conferred with GRADE group members who were available at the first committee meeting and subsequent nominal group meetings. GRADE representatives were also used as a resource throughout subgroup deliberation.

The GRADE system is based on a sequential assessment of the quality of evidence, followed by assessment of the balance between benefits versus risks, burden, and cost and, based on the above, development and grading of a management recommendations [9-11]. Keeping the rating of quality of evidence and strength of recommendation explicitly separate constitutes a crucial and defining feature of the GRADE approach. This system classifies quality of evidence as high (Grade A), moderate (Grade B), low (Grade C), or very low (Grade D). Randomized trials begin as high quality evidence, but may be downgraded due to limitations in implementation, inconsistency or imprecision of the results, indirectness of the evidence, and possible reporting bias (see Table 1). Examples of indirectness of the evidence include: population studied, interventions used, outcomes measured, and how these relate to the question of interest. Observational (non-randomized) studies begin as low-quality evidence, but the quality level may be upgraded on the basis of large magnitude of effect. An example of this is the quality of evidence for early administration of antibiotics.

The GRADE system classifies recommendations as strong (Grade 1) or weak (Grade 2). The grade of strong or weak is considered of greater clinical importance than a difference in letter level of quality of evidence. The committee assessed whether the desirable effects of adherence will outweigh the undesirable effects, and the strength of a recommendation reflects the group's degree of confidence in that assessment. A strong recommendation in favor of an intervention reflects that the desirable effects of adherence to a recommendation (beneficial health outcomes, less burden on staff and patients, and cost savings) will clearly outweigh the undesirable effects (harms, more burden and greater costs). A weak recommendation in favor of an intervention indicates that the desirable effects of adherence to a recommendation probably will outweigh the undesirable effects, but the panel is not confident about these tradeoffs - either because some of the evidence is low-quality (and thus there remains uncertainty regarding the benefits and risks) or the benefits and downsides are closely balanced. While the degree of confidence is a continuum and there is a lack of a precise threshold between a strong and a weak recommendation, the presence of important concerns about one or more of the above factors makes a weak recommendation more likely. A "strong" recommendation is worded as "we recommend" and a weak recommendation as "we suggest."

The implications of calling a recommendation "strong" are that most well-informed patients would accept that intervention, and that most clinicians should use it in most situations. There may be circumstances in which a "strong" recommendation cannot or should not be followed for an individual patient because of that patient's preferences or clinical characteristics which make the recommendation less applicable. It should be noted that being a "strong" recommendation does not automatically imply standard of care. For example, the strong recommendation for administering antibiotics within one hour of the diagnosis of severe sepsis, although desirable, is not currently standard of care as verified by current practice (personal communication, Mitchell Levy from first 8,000 patients entered internationally into the SSC performance improvement data base). The implication of a "weak" recommendation is that although a majority of well-informed patients would accept it (but a substantial proportion would not), clinicians should consider its use according to particular circumstance.

Differences of opinion among committee members about interpretation of evidence, wording of proposals, or strength of recommendations were resolved using a specifically developed set of rules. We will describe this process in detail in a separate publication. In summary, the main approach for converting diverse opinions into a recommendation was: 1 . to give a recommendation a direction (for or against the given action). a majority of votes were to be in favor of that direction, with no more than $20 \%$ preferring the opposite direction (there was a neutral vote allowed as well); 2. to call a given recommendation "strong" rather than "weak" at least $70 \%$ "strong" votes were required; 3. if fewer than $70 \%$ of votes indicated "strong" preference, the recommendation was assigned a "weak" category of strength. We used a combination of modified Delphi Process and Nominal (Expert) Group techniques to ensure both depth and breadth of review. The entire review group (together with their parent organizations as required) participated in the larger, iterative, modified Delphi process. The smaller working group meetings which took place in person functioned as the Nominal Groups. If a clear consensus could not be obtained by polling within the Nominal Group meetings, the larger group was specifically asked to use the polling process. This was only required for corticosteroids and glycemic control. The larger group had the opportunity to review all outputs. In this way the entire review combined intense focused discussion (Nominal Group) with broader review and monitoring using the Delphi process. 


\section{Note: Refer to Tables 3, 4, and 5 for condensed adult recommentations.}

\section{Management of Severe Sepsis}

\section{A. Initial Resuscitation}

1. We recommend the protocolized resuscitation of a patient with sepsis-induced shock, defined as tissue hypoperfusion (hypotension persisting after initial fluid challenge or blood lactate concentration equal to or greater than $4 \mathrm{mmol} / \mathrm{L}$ ). This protocol should be initiated as soon as hypoperfusion is recognized and should not be delayed pending ICU admission. During the first $6 \mathrm{hrs}$ of resuscitation, the goals of initial resuscitation of sepsis-induced hypoperfusion should include all of the following as one part of a treatment protocol:

Central venous pressure (CVP): $8-12 \mathrm{~mm} \mathrm{Hg}$ Mean arterial pressure (MAP) $\geq 65 \mathrm{~mm} \mathrm{Hg}$ Urine output $\geq 0.5 \mathrm{~mL} \cdot \mathrm{kg}^{-1} \cdot \mathrm{hr}^{-1}$

Central venous (superior vena cava) or mixed venous oxygen saturation $\geq 70 \%$ or $\geq 65 \%$, respectively (Grade 1C)

Rationale. Early goal-directed resuscitation has been shown to improve survival for emergency department patients presenting with septic shock in a randomized, controlled, single-center study [16]. Resuscitation directed toward the previously mentioned goals for the initial 6-hr period of the resuscitation was able to reduce 28-day mortality rate. The consensus panel judged use

Table 3 Initial Resuscitation and Infection Issues

\section{Initial resuscitation (first 6 hours)}

Strength of recommendation and quality of evidence have been assessed using the GRADE criteria, presented in brackets after each guideline. For added clarity: • Indicates a strong recommendation or "we recommend"; o indicates a weak recommendation or "we suggest"

- Begin resuscitation immediately in patients with hypotension or elevated serum lactate $>4 \mathrm{mmol} / \mathrm{l}$; do not delay pending ICU admission. (1C)

- Resuscitation goals: $(1 C)$

- Central venous pressure (CVP) $8-12 \mathrm{~mm} \mathrm{Hg} *$

- Mean arterial pressure $\geq 65 \mathrm{~mm} \mathrm{Hg}$

- Urine output $\geq 0.5 \mathrm{~mL} . \mathrm{kg}-1 . \mathrm{hr}-1$

- Central venous (superior vena cava) oxygen saturation $\geq 70 \%$, or mixed venous $\geq 65 \%$

- If venous $\mathrm{O}_{2}$ saturation target not achieved: $(2 \mathrm{C})$

- consider further fluid

- transfuse packed red blood cells if required to hematocrit of $\geq 30 \%$ and/or

- dobutamine infusion max $20 \mu \mathrm{g} \cdot \mathrm{kg}^{-1} \cdot \mathrm{min}^{-1}$

* A higher target CVP of $12-15 \mathrm{mmHg}$ is recommended in the presence of mechanical ventilation or pre-existing decreased

ventricular compliance.

Diagnosis

- Obtain appropriate cultures before starting antibiotics provided this does not significantly delay antimicrobial administration. (1C)

- Obtain two or more blood cultures (BCs)

- One or more BCs should be percutaneous

- One BC from each vascular access device in place $>48 \mathrm{~h}$

- Culture other sites as clinically indicated

- Perform imaging studies promptly in order to confirm and sample any source of infection; if safe to do so. (1C)

Antibiotic therapy

- Begin intravenous antibiotics as early as possible, and always within the first hour of recognizing severe sepsis $(1 D)$ and septic shock $(1 B)$.

- Broad-spectrum: one or more agents active against likely bacterial/fungal pathogens and with good penetration into presumed source. $(1 B)$

- Reassess antimicrobial regimen daily to optimise efficacy, prevent resistance, avoid toxicity \& minimise costs. (1C)

- Consider combination therapy in Pseudomonas infections. $(2 D)$

- Consider combination empiric therapy in neutropenic patients. $(2 D)$

- Combination therapy no more than 3-5 days and deescalation following susceptibilities. $(2 D)$

- Duration of therapy typically limited to 7-10 days; longer if response slow, undrainable foci of infection,

or immunologic deficiencies. $(1 D)$

- Stop antimicrobial therapy if cause is found to be non-infectious. $(1 D)$

Source identification and control

- A specific anatomic site of infection should be established as rapidly as possible ${ }_{(1 C)}$ and within first 6 hrs of presentation $(1 D)$.

- Formally evaluate patient for a focus of infection amenable to source control measures (eg: abscess drainage, tissue debridement). (1C)

- Implement source control measures as soon as possible following successful initial resuscitation. (1C)

Exception: infected pancreatic necrosis, where surgical intervention best delayed. $(2 B)$

- Choose source control measure with maximum efficacy and minimal physiologic upset. $(1 D)$

- Remove intravascular access devices if potentially infected. (1C) 
Table 4 Hemodynamic Support and Adjunctive Therapy

\section{Fluid therapy}

Strength of recommendation and quality of evidence have been assessed using the GRADE criteria, presented in brackets after each guide-

line. For added clarity: • Indicates a strong recommendation or "we recommend"; o indicates a weak recommendation or "we suggest"

- Fluid-resuscitate using crystalloids or colloids. $(1 B)$

- Target a CVP of $\geq 8 \mathrm{~mm} \mathrm{Hg}$ ( $\geq 12 \mathrm{~mm} \mathrm{Hg}$ if mechanically ventilated). (1C)

- Use a fluid challenge technique while associated with a haemodynamic improvement. $(1 D)$

- Give fluid challenges of $1000 \mathrm{ml}$ of crystalloids or 300-500 $\mathrm{ml}$ of colloids over $30 \mathrm{~min}$. More rapid and larger volumes may be required in sepsis-induced tissue hypoperfusion. $(1 D)$

- Rate of fluid administration should be reduced if cardiac filling pressures increase without concurrent hemodynamic improvement. (1D)

Vasopressors

- Maintain MAP $\geq 65 \mathrm{~mm} \mathrm{Hg}$. (1C)

- Norepinephrine or dopamine centrally administered are the initial vasopressors of choice. (1C)

- Epinephrine, phenylephrine or vasopressin should not be administered as the initial vasopressor in septic shock. (2C)

- Vasopressin 0.03 units/min maybe subsequently added to norepinephrine with anticipation of an effect equivalent to norepinephrine alone.

- Use epinephrine as the first alternative agent in septic shock when blood pressure is poorly responsive to norepinephrine

or dopamine. $(2 B)$

- Do not use low-dose dopamine for renal protection. $(1 \mathrm{~A})$

- In patients requiring vasopressors, insert an arterial catheter as soon as practical. $(1 D)$

Inotropic therapy

- Use dobutamine in patients with myocardial dysfunction as supported by elevated cardiac filling pressures and low cardiac output. (1C)

- Do not increase cardiac index to predetermined supranormal levels. (1B)

Steroids

- Consider intravenous hydrocortisone for adult septic shock when hypotension remains poorly responsive to adequate

fluid resuscitation and vasopressors. $(2 \mathrm{C})$

- ACTH stimulation test is not recommended to identify the subset of adults with septic shock who should receive hydrocortisone. (2B)

- Hydrocortisone is preferred to dexamethasone. $(2 B)$

- Fludrocortisone $(50 \mu \mathrm{g}$ orally once a day) may be included if an alternative to hydrocortisone is being used which lacks significant

mineralocorticoid activity. Fludrocortisone is optional if hydrocortisone is used. $(2 C)$

- Steroid therapy may be weaned once vasopressors are no longer required. $(2 D)$

- Hydrocortisone dose should be $\leq 300 \mathrm{mg} /$ day. (1A)

- Do not use corticosteroids to treat sepsis in the absence of shock unless the patient's endocrine or corticosteroid history warrants it. $(1 D)$

Recombinant human activated protein $\mathrm{C}$ (rhAPC)

- Consider rhAPC in adult patients with sepsis-induced organ dysfunction with clinical assessment of high risk of death

(typically APACHE II $\geq 25$ or multiple organ failure) if there are no contraindications. ( $2 B, 2$ Cfor post-operative patients)

- Adult patients with severe sepsis and low risk of death (e. g.: APACHE II $<20$ or one organ failure) should not receive rhAPC. (1A)

of central venous and mixed venous oxygen saturation targets to be equivalent. Either intermittent or continuous measurements of oxygen saturation were judged to be acceptable. Although blood lactate concentration may lack precision as a measure of tissue metabolic status, elevated levels in sepsis support aggressive resuscitation. In mechanically ventilated patients or patients with known pre-existing decreased ventricular compliance, a higher target CVP of $12-15 \mathrm{~mm} \mathrm{Hg}$ is recommended to account for the impediment to filling [17]. Similar consideration may be warranted in circumstances of increased abdominal pressure or diastolic dysfunction [18]. Elevated central venous pressures may also be seen with pre-existing clinically significant pulmonary artery hypertension. Although the cause of tachycardia in septic patients may be multifactorial, a decrease in elevated pulse rate with fluid resuscitation is often a useful marker of improving intravascular filling. Recently published observational studies have demonstrated an association between good clinical outcome in septic shock and MAP $\geq 65 \mathrm{~mm} \mathrm{Hg}$ as well as central venous oxygen saturation $\left(\mathrm{ScvO}_{2}\right.$, measured in superior vena cava, either intermittently or continuously) of $\geq 70 \%$ [19]. Many recent studies support the value of early protocolized resuscitation in severe sepsis and sepsis-induced tissue hypoperfusion [20-25]. Studies of patients with shock indicate that $\mathrm{SvO}_{2}$ runs 5-7\% lower than central venous oxygen saturation $\left(\mathrm{ScvO}_{2}\right)$ [26] and that an early goal directed resuscitation protocol can be established in a non-research general practice venue [27].

There are recognized limitations to ventricular filling pressure estimates as surrogates for fluid resuscitation [28, 29]. However, measurement of CVP is currently the most readily obtainable target for fluid resuscitation. There may be advantages to targeting fluid resuscitation to flow and perhaps to volumetric indices (and even to microcirculation changes) [30-33]. Technologies currently exist that allow measurement of flow at the bedside [34, 35]. Future goals should be making these technologies more accessible during the critical early resuscitation period and research to validate utility. These technologies are already available for early ICU resuscitation. 
Table 5 Other Supportive Therapy of Severe Sepsis

\section{Blood product administration}

Strength of recommendation and quality of evidence have been assessed using the GRADE criteria, presented in brackets after each guideline. For added clarity: • Indicates a strong recommendation or "we recommend"; o indicates a weak recommendation or "we suggest"

- Give red blood cells when hemoglobin decreases to $<7.0 \mathrm{~g} / \mathrm{dl}(<70 \mathrm{~g} / \mathrm{L})$ to target a hemoglobin of 7.0-9.0 g/dl in adults. (1B)

- A higher hemoglobin level may be required in special circumstances (e. g.: myocardial ischaemia, severe hypoxemia, acute haemorrhage, cyanotic heart disease or lactic acidosis)

- Do not use erythropoietin to treat sepsis-related anemia. Erythropoietin may be used for other accepted reasons. $(1 B)$

- Do not use fresh frozen plasma to correct laboratory clotting abnormalities unless there is bleeding or planned invasive procedures. $(2 D)$

o Do not use antithrombin therapy. $(1 B)$

- Administer platelets when: $(2 D)$

- counts are $<5000 / \mathrm{mm}^{3}\left(5 \times 10^{9} / \mathrm{L}\right)$ regardless of bleeding.

- counts are 5000 to $30,000 / \mathrm{mm}^{3}\left(5-30 \times 10^{9} / \mathrm{L}\right)$ and there is significant bleeding risk.

- Higher platelet counts $\left(\geq 50,000 / \mathrm{mm}^{3}\left(50 \times 10^{9} / \mathrm{L}\right)\right)$ are required for surgery or invasive procedures.

Mechanical ventilation of sepsis-induced acute lung injury (ALI)/ARDS

- Target a tidal volume of $6 \mathrm{ml} / \mathrm{kg}$ (predicted) body weight in patients with ALI/ARDS. $(1 B)$

- Target an initial upper limit plateau pressure $\leq 30 \mathrm{~cm} \mathrm{H}_{2} \mathrm{O}$. Consider chest wall compliance when assessing plateau pressure. (1C)

- Allow $\mathrm{PaCO}_{2}$ to increase above normal, if needed to minimize plateau pressures and tidal volumes. (1C)

- Positive end expiratory pressure (PEEP) should be set to avoid extensive lung collapse at end expiration. $(1 C)$

- Consider using the prone position for ARDS patients requiring potentially injurious levels of $\mathrm{FiO}_{2}$ or plateau pressure, provided they are not put at risk from positional changes. $(2 C)$

- Maintain mechanically ventilated patients in a semi-recumbent position (head of the bed raised to $45^{\circ}$ ) unless contraindicated $(1 B)$, between $30^{\circ}-45^{\circ}(2 C)$.

- Non invasive ventilation may be considered in the minority of ALI/ARDS patients with mild-moderate hypoxemic respiratory failure.

The patients need to be hemodynamically stable, comfortable, easily arousable, able to protect/clear their airway and expected

to recover rapidly. $(2 B)$

- Use a weaning protocol and a spontaneous breathing trial (SBT) regularly to evaluate the potential for discontinuing mechanical ventilation. $(1 \mathrm{~A})$

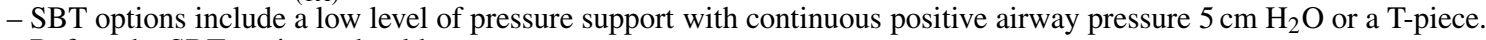

- Before the SBT, patients should:

- be arousable

- be haemodynamically stable without vasopressors

- have no new potentially serious conditions

- have low ventilatory and end-expiratory pressure requirement

- require $\mathrm{FiO}_{2}$ levels that can be safely delivered with a face mask or nasal cannula

- Do not use a pulmonary artery catheter for the routine monitoring of patients with ALI/ARDS. (1A)

- Use a conservative fluid strategy for patients with established ALI who do not have evidence of tissue hypoperfusion. (1C)

Sedation, analgesia, and neuromuscular blockade in sepsis

- Use sedation protocols with a sedation goal for critically ill mechanically ventilated patients. $(1 B)$

- Use either intermittent bolus sedation or continuous infusion sedation to predetermined end points (sedation scales), with daily

interruption/lightening to produce awakening. Re-titrate if necessary. $(1 B)$

- Avoid neuromuscular blockers (NMBs) where possible. Monitor depth of block with train of four when using continuous infusions. (1B)

Glucose control

- Use IV insulin to control hyperglycemia in patients with severe sepsis following stabilization in the ICU. (1B)

- Aim to keep blood glucose $<150 \mathrm{mg} / \mathrm{dl}(8.3 \mathrm{mmol} / \mathrm{L})$ using a validated protocol for insulin dose adjustment. $(2 C)$

- Provide a glucose calorie source and monitor blood glucose values every 1-2 hrs (4 hrs when stable) in patients receiving intravenous insulin. $(1 C)$

- Interpret with caution low glucose levels obtained with point of care testing, as these techniques may overestimate arterial blood

or plasma glucose values. $(1 B)$

Renal replacement

o Intermittent hemodialysis and continuous veno-venous haemofiltration $(\mathrm{CVVH})$ are considered equivalent. $(2 B)$

$\circ$ CVVH offers easier management in hemodynamically unstable patients. $(2 D)$

Bicarbonate therapy

- Do not use bicarbonate therapy for the purpose of improving hemodynamics or reducing vasopressor requirements when treating hypoperfusion-induced lactic acidemia with $\mathrm{pH} \geq 7.15$. (1B)

Deep vein thrombosis (DVT) prophylaxis

- Use either low-dose unfractionated heparin (UFH) or low-molecular weight heparin (LMWH), unless contraindicated. (1A)

- Use a mechanical prophylactic device, such as compression stockings or an intermittent compression device, when heparin is contraindicated. $(1 \mathrm{~A})$

- Use a combination of pharmacologic and mechanical therapy for patients who are at very high risk for DVT. $(2 C)$

$\circ$ In patients at very high risk LMWH should be used rather than UFH. $(2 C)$

Stress ulcer prophylaxis

- Provide stress ulcer prophylaxis using $\mathrm{H}_{2}$ blocker $(1 \mathrm{~A})$ or proton pump inhibitor $(1 \mathrm{~B})$. Benefits of prevention of upper GI bleed must

be weighed against the potential for development of ventilator-associated pneumonia.

Consideration for limitation of support

- Discuss advance care planning with patients and families. Describe likely outcomes and set realistic expectations. $(1 D)$ 
2. We suggest that during the first $6 \mathrm{hrs}$ of resuscitation of severe sepsis or septic shock, if $\mathrm{S}_{\mathrm{CV}} \mathrm{O}_{2}$ or $\mathrm{SvO}_{2}$ of $70 \%$ or $65 \%$ respectively is not achieved with fluid resuscitation to the CVP target, then transfusion of packed red blood cells to achieve a hematocrit of $\geq 30 \%$ and/or administration of a dobutamine infusion (up to a maximum of $20 \mu \mathrm{g} \cdot \mathrm{kg}^{-1} \cdot \mathrm{min}^{-1}$ ) be utilized to achieve this goal (Grade 2C).

Rationale. The protocol used in the study cited previously targeted an increase in $\mathrm{S}_{\mathrm{CV}} \mathrm{O}_{2}$ to $\geq 70 \%$ [16]. This was achieved by sequential institution of initial fluid resuscitation, then packed red blood cells, and then dobutamine. This protocol was associated with an improvement in survival. Based on bedside clinical assessment and personal preference, a clinician may deem either blood transfusion (if Hct is less than 30\%) or dobutamine the best initial choice to increase oxygen delivery and thereby elevate $\mathrm{S}_{\mathrm{CV}} \mathrm{O}_{2}$. When fluid resuscitation is believed to be already adequate. The design of the afore mentioned trial did not allow assessment of the relative contribution of these two components (i. e. increasing $\mathrm{O}_{2}$ content or increasing cardiac output) of the protocol on achievement of improved outcome.

\section{B. Diagnosis}

1. We recommend obtaining appropriate cultures before antimicrobial therapy is initiated if such cultures do not cause significant delay in antibiotic administration. To optimize identification of causative organisms, we recommend at least two blood cultures be obtained prior to antibiotics with at least one drawn percutaneously and one drawn through each vascular access device, unless the device was recently $(<48 \mathrm{~h})$ inserted. Cultures of other sites (preferably quantitative where appropriate) such as urine, cerebrospinal fluid, wounds, respiratory secretions, or other body fluids that may be the source of infection should also be obtained before antibiotic therapy if not associated with significant delay in antibiotic administration (Grade 1C).

Rationale. Although sampling should not delay timely administration of antibiotics in patients with severe sepsis (example: lumbar puncture in suspected meningitis), obtaining appropriate cultures prior to their administration is essential to confirm infection and the responsible pathogen(s), and to allow de-escalation of antibiotic therapy after receipt of the susceptibility profile. Samples can be kept in the refrigerator or frozen if processing cannot be performed immediately. Immediate transport to a microbiological lab is necessary. Because rapid sterilization of blood cultures can occur within a few hours after the first antibiotic dose, obtaining those cultures before starting therapy is essential if the causative organism is to be identified. Two or more blood cultures are recommended [36]. In patients with indwelling catheters (for $>48 \mathrm{~h}$ ) at least one blood culture should be drawn through each lumen of each vascular access device. Obtaining blood cultures peripherally and through a vascular access device is an important strategy. If the same organism is recovered from both cultures, the likelihood that the organism is causing the severe sepsis is enhanced. In addition, if the culture drawn through the vascular access device is positive much earlier than the peripheral blood culture (i.e., $>2 \mathrm{hrs}$ earlier), the data support the concept that the vascular access device is the source of the infection [37]. Quantitative cultures of catheter and peripheral blood are also useful for determining whether the catheter is the source of infection. Volume of blood drawn with the culture tube should be at least $10 \mathrm{~mL}$ [38]. Quantitative (or semi-quantitative) cultures of respiratory tract secretions are recommended for the diagnosis of ventilator-associated pneumonia [39]. Gram stain can be useful, in particular for respiratory tract specimens, to help decide the micro-organisms to be targeted. The potential role of biomarkers for diagnosis of infection in patients presenting with severe sepsis remains at present undefined. The procalcitonin level, although often useful, is problematic in patients with an acute inflammatory pattern from other causes (e. g. post-operative, shock) [40] In the near future, rapid diagnostic methods (polymerase chain reaction, micro-arrays) might prove extremely helpful for a quicker identification of pathogens and major antimicrobial resistance determinants [41].

2. We recommend that imaging studies be performed promptly in attempts to confirm a potential source of infection. Sampling of potential sources of infection should occur as they are identified; however, some patients may be too unstable to warrant certain invasive procedures or transport outside of the ICU. Bedside studies, such as ultrasound, are useful in these circumstances (Grade 1C).

Rationale. Diagnostic studies may identify a source of infection that requires removal of a foreign body or drainage to maximize the likelihood of a satisfactory response to therapy. However, even in the most organized and well-staffed healthcare facilities, transport of patients can be dangerous, as can placing patients in outside-unit imaging devices that are difficult to access and monitor. Balancing risk and benefit is therefore mandatory in those settings.

\section{Antibiotic Therapy}

1. We recommend that intravenous antibiotic therapy be started as early as possible and within the first hour of recognition of septic shock (1B) and severe sepsis without septic shock (1D). Appropriate cultures 
should be obtained before initiating antibiotic therapy, but should not prevent prompt administration of antimicrobial therapy (Grade 1D).

Rationale. Establishing vascular access and initiating aggressive fluid resuscitation is the first priority when managing patients with severe sepsis or septic shock. However, prompt infusion of antimicrobial agents should also be a priority and may require additional vascular access ports $[42,43]$. In the presence of septic shock each hour delay in achieving administration of effective antibiotics is associated with a measurable increase in mortality [42]. If antimicrobial agents cannot be mixed and delivered promptly from the pharmacy, establishing a supply of premixed antibiotics for such urgent situations is an appropriate strategy for ensuring prompt administration. In choosing the antimicrobial regimen, clinicians should be aware that some antimicrobial agents have the advantage of bolus administration, while others require a lengthy infusion. Thus, if vascular access is limited and many different agents must be infused, bolus drugs may offer an advantage.

2a. We recommend that initial empirical anti-infective therapy include one or more drugs that have activity against all likely pathogens (bacterial and/or fungal) and that penetrate in adequate concentrations into the presumed source of sepsis (Grade 1B).

Rationale. The choice of empirical antibiotics depends on complex issues related to the patient's history including drug intolerances, underlying disease, the clinical syndrome, and susceptibility patterns of pathogens in the community, in the hospital, and that previously have been documented to colonize or infect the patient. There is an especially wide range of potential pathogens for neutropenic patients.

Recently used antibiotics should generally be avoided. Clinicians should be cognizant of the virulence and growing prevalence of oxacillin (methicillin) resistant Staphylococcus aureus (ORSA or MRSA) in some communities and healthcare associated settings (especially in the United States) when they choose empiric therapy. If the prevalence is significant, and in consideration of the virulence of this organism, empiric therapy adequate for this pathogen would be warranted. Clinicians should also consider whether Candidemia is a likely pathogen when choosing initial therapy. When deemed warranted, the selection of empiric antifungal therapy (e. g., fluconazole, amphotericin B, or echinocandin) will be tailored to the local pattern of the most prevalent Candida species, and any prior administration of azoles drugs [44]. Risk factors for candidemia should also be considered when choosing initial therapy.

Because patients with severe sepsis or septic shock have little margin for error in the choice of therapy, the initial selection of antimicrobial therapy should be broad enough to cover all likely pathogens. There is ample evidence that failure to initiate appropriate therapy (i. e. therapy with activity against the pathogen that is subsequently identified as the causative agent) correlates with increased morbidity and mortality [45-48].

Patients with severe sepsis or septic shock warrant broad-spectrum therapy until the causative organism and its antibiotic susceptibilities are defined. Restriction of antibiotics as a strategy to reduce the development of antimicrobial resistance or to reduce cost is not an appropriate initial strategy in this patient population.

All patients should receive a full loading dose of each antimicrobial. However, patients with sepsis or septic shock often have abnormal renal or hepatic function and may have abnormal volumes of distribution due to aggressive fluid resuscitation. Drug serum concentration monitoring can be useful in an ICU setting for those drugs that can be measured promptly. An experienced physician or clinical pharmacist should be consulted to ensure that serum concentrations are attained that maximize efficacy and minimize toxicity [49-52].

$2 \mathrm{~b}$. We recommend that the antimicrobial regimen be reassessed daily to optimize activity, to prevent the development of resistance, to reduce toxicity, and to reduce costs (Grade 1C).

Rationale. Although restriction of antibiotics as a strategy to reduce the development of antimicrobial resistance or to reduce cost is not an appropriate initial strategy in this patient population, once the causative pathogen has been identified, it may become apparent that none of the empiric drugs offers optimal therapy; i. e., there may be another drug proven to produce superior clinical outcome which should therefore replace empiric agents.

Narrowing the spectrum of antibiotic coverage and reducing the duration of antibiotic therapy will reduce the likelihood that the patient will develop superinfection with pathogenic or resistant organisms such as Candida species, Clostridium difficile, or vancomycin-resistant Enterococcus faecium. However, the desire to minimize superinfections and other complications should not take precedence over the need to give the patient an adequate course of therapy to cure the infection that caused the severe sepsis or septic shock.

2c. We suggest combination therapy for patients with known or suspected Pseudomonas infections as a cause of severe sepsis (Grade 2D).

$2 d$. We suggest combination empiric therapy for neutropenic patients with severe sepsis (Grade 2D).

2e. When used empirically in patients with severe sepsis, we suggest that combination therapy should not be administered for more than 3 to 5 days. De-escalation 
to the most appropriate single therapy should be performed as soon as the susceptibility profile is known. (Grade 2D).

Rationale. Although no study or meta-analysis has convincingly demonstrated that combination therapy produces a superior clinical outcome for individual pathogens in a particular patient group, combination therapies do produce in vitro synergy against pathogens in some models (although such synergy is difficult to define and predict). In some clinical scenarios, such as the two above, combination therapies are biologically plausible and are likely clinically useful even if evidence has not demonstrated improved clinical outcome [53-56]. Combination therapy for suspected known Pseudomonas pending sensitivities increases the likelihood that at least one drug is effective against that strain and positively affects outcome [57].

3. We recommend that the duration of therapy typically be 7-10 days; longer courses may be appropriate in patients who have a slow clinical response, undrainable foci of infection, or who have immunologic deficiencies including neutropenia (Grade 1D).

4. If the presenting clinical syndrome is determined to be due to a noninfectious cause, we recommend antimicrobial therapy be stopped promptly to minimize the likelihood that the patient will become infected with an antibiotic resistant pathogen or will develop a drug related adverse effect (Grade 1D).

Rationale. Clinicians should be cognizant that blood cultures will be negative in more than $50 \%$ of cases of severe sepsis or septic shock, yet many of these cases are very likely caused by bacteria or fungi. Thus, the decisions to continue, narrow, or stop antimicrobial therapy must be made on the basis of clinician judgment and clinical information.

\section{Source Control}

1a. We recommend that a specific anatomic diagnosis of infection requiring consideration for emergent source control- for example necrotizing fasciitis, diffuse peritonitis, cholangitis, intestinal infarction - be sought and diagnosed or excluded as rapidly as possible (Grade 1C) and within the first 6 hours following presentation (Grade 1D).

1b. We further recommend that all patients presenting with severe sepsis be evaluated for the presence of a focus of infection amenable to source control measures, specifically the drainage of an abscess or local focus of infection, the debridement of infected necrotic tissue, the removal of a potentially infected device, or the definitive control of a source of ongoing microbial contamination (Grade 1C) (see Appendix A for examples of potential sites needing source control).

2. We suggest that when infected peripancreatic necrosis is identified as a potential source of infection, definitive intervention is best delayed until adequate demarcation of viable and non-viable tissues has occurred (Grade 2B).

3. We recommend that when source control is required, the effective intervention associated with the least physiologic insult be employed e.g., percutaneous rather than surgical drainage of an abscess (Grade 1D).

4. We recommend that when intravascular access devices are a possible source of severe sepsis or septic shock, they be promptly removed after establishing other vascular access (Grade 1C).

Rationale. The principles of source control in the management of sepsis include a rapid diagnosis of the specific site of infection, and identification of a focus of infection amenable to source control measures (specifically the drainage of an abscess, the debridement of infected necrotic tissue, the removal of a potentially infected device, and the definitive control of a source of ongoing microbial contamination) [58]. Foci of infection readily amenable to source control measures include an intra-abdominal abscess or gastrointestinal perforation, cholangitis or pyelonephritis, intestinal ischemia or necrotizing soft tissue infection, and other deep space infection such as an empyema or septic arthritis. Such infectious foci should be controlled as soon as possible following successful initial resuscitation [59], accomplishing the source control objective with the least physiologic upset possible (e. g., percutaneous rather than surgical drainage of an abscess [60], endoscopic rather than surgical drainage of biliary tree), and removing intravascular access devices that are potentially the source of severe sepsis or septic shock promptly after establishing other vascular access [61,62]. A randomized, controlled trial comparing early vs. delayed surgical intervention for peripancreatic necrosis showed better outcomes with a delayed approach [63]. However, areas of uncertainty, such as definitive documentation of infection and appropriate length of delay exist. The selection of optimal source control methods must weigh benefits and risks of the specific intervention as well as risks of transfer [64]. Source control interventions may cause further complications such as bleeding, fistulas, or inadvertent organ injury. Surgical intervention should be considered when lesser interventional approaches are inadequate, or when diagnostic uncertainty persists despite radiological evaluation. Specific clinical situations require consideration of available choices, patient's preferences, and clinician's expertise. 


\section{E. Fluid Therapy}

1. We recommend fluid resuscitation with either natural/artificial colloids or crystalloids. There is no evidence-based support for one type of fluid over another (Grade 1B).

Rationale. The SAFE study indicated albumin administration was safe and equally effective as crystalloid [65]. There was an insignificant decrease in mortality rates with the use of colloid in a subset analysis of septic patients $(p=0.09)$. Previous meta-analyses of small studies of ICU patients had demonstrated no difference between crystalloid and colloid fluid resuscitation [66-68]. Although administration of hydroxyethyl starch may increase the risk of acute renal failure in patients with sepsis variable findings preclude definitive recommendations $[69,70]$. As the volume of distribution is much larger for crystalloids than for colloids, resuscitation with crystalloids requires more fluid to achieve the same end points and results in more edema. Crystalloids are less expensive.

2. We recommend fluid resuscitation initially target a CVP of at least $8 \mathrm{~mm} \mathrm{Hg}(12 \mathrm{~mm} \mathrm{Hg}$ in mechanically ventilated patients). Further fluid therapy is often required (Grade 1C).

3a. We recommend that a fluid challenge technique be applied, wherein fluid administration is continued as long as the hemodynamic improvement (e.g., arterial pressure, heart rate, urine output) continues (Grade 1D)

3b. We recommend fluid challenge in patients with suspected hypovolemia be started with at least $1000 \mathrm{~mL}$ of crystalloids or $300-500 \mathrm{~mL}$ of colloids over $30 \mathrm{~min}$. More rapid administration and greater amounts of fluid may be needed in patients with sepsis induced tissue hypoperfusion (see initial resuscitation recommendations) (Grade 1D).

3c. We recommend the rate of fluid administration be reduced substantially when cardiac filling pressures (CVP or pulmonary artery balloon-occluded pressure) increase without concurrent hemodynamic improvement (Grade 1D).

Rationale. Fluid challenge must be clearly separated from simple fluid administration; it is a technique in which large amounts of fluids are administered over a limited period of time under close monitoring to evaluate the patient's response and avoid the development of pulmonary edema. The degree of intravascular volume deficit in patients with severe sepsis varies. With venodilation and ongoing capillary leak, most patients require continuing aggressive fluid resuscitation during the first 24 hours of management. Input is typically much greater than output, and input/output ratio is of no utility to judge fluid resuscitation needs during this time period.

\section{F. Vasopressors}

1. We recommend mean arterial pressure (MAP) be maintained $\geq 65 \mathrm{~mm} \mathrm{Hg}$ (Grade 1C).

Rationale. Vasopressor therapy is required to sustain life and maintain perfusion in the face of life-threatening hypotension, even when hypovolemia has not yet been resolved. Below a certain mean arterial pressure, autoregulation in various vascular beds can be lost, and perfusion can become linearly dependent on pressure. Thus, some patients may require vasopressor therapy to achieve a minimal perfusion pressure and maintain adequate flow [71, 72]. The titration of norepinephrine to as low as MAP $65 \mathrm{~mm} \mathrm{Hg}$ has been shown to preserve tissue perfusion [72]. In addition, pre-existing comorbidities should be considered as to most appropriate MAP target. For example, a MAP of $65 \mathrm{~mm} \mathrm{Hg}$ might be too low in a patient with severe uncontrolled hypertension, and in a young previously normotensive, a lower MAP might be adequate. Supplementing end points such as blood pressure with assessment of regional and global perfusion, such as blood lactate concentrations and urine output, is important. Adequate fluid resuscitation is a fundamental aspect of the hemodynamic management of patients with septic shock, and should ideally be achieved before vasopressors and inotropes are used, but using vasopressors early as an emergency measure in patients with severe shock is frequently necessary. When that occurs great effort should be directed to weaning vasopressors with continuing fluid resuscitation.

2. We recommend either norepinephrine or dopamine as the first choice vasopressor agent to correct hypotension in septic shock (administered through a central catheter as soon as one is available) (Grade 1C).

3a. We suggest that epinephrine, phenylephrine, or vasopressin should not be administered as the initial vasopressor in septic shock (Grade 2C). Vasopressin .03 units/min may be subsequently added to norepinephrine with anticipation of an effect equivalent to norepinephrine alone.

$3 \mathrm{~b}$. We suggest that epinephrine be the first chosen alternative agent in septic shock that is poorly responsive to norepinephrine or dopamine (Grade $2 \mathrm{~B}$ ).

Rationale. There is no high-quality primary evidence to recommend one catecholamine over another. Much literature exists that contrasts the physiologic effects of choice of vasopressor and combined inotrope/vasopressors in septic shock [73-85]. Human and animal studies suggest some advantages of norepinephrine and dopamine over epinephrine (the latter with the potential for tachycardia as well as disadvantageous effects on splanchnic circulation and hyperlactemia) and phenylephrine (decrease in stroke volume). There is, however, no clinical evidence that 
epinephrine results in worse outcomes, and it should be the first chosen alternative to dopamine or norepinephrine. Phenylephrine is the adrenergic agent least likely to produce tachycardia, but as a pure vasopressor would be expected to decrease stroke volume. Dopamine increases mean arterial pressure and cardiac output, primarily due to an increase in stroke volume and heart rate. Norepinephrine increases mean arterial pressure due to its vasoconstrictive effects, with little change in heart rate and less increase in stroke volume compared with dopamine. Either may be used as a first-line agent to correct hypotension in sepsis. Norepinephrine is more potent than dopamine and may be more effective at reversing hypotension in patients with septic shock. Dopamine may be particularly useful in patients with compromised systolic function but causes more tachycardia and may be more arrhythmogenic [86]. It may also influence the endocrine response via the hypothalamic-pituitary axis and have immunosuppressive effects.

Vasopressin levels in septic shock have been reported to be lower than anticipated for a shock state [87]. Low doses of vasopressin may be effective in raising blood pressure in patients refractory to other vasopressors, and may have other potential physiologic benefits [88-93]. Terlipressin has similar effects but is long lasting [94]. Studies show that vasopressin concentrations are elevated in early septic shock, but with continued shock, concentration decreases to normal range in the majority of patients between 24 and $48 \mathrm{hrs}$ [95]. This has been called "relative vasopressin deficiency" because in the presence of hypotension, vasopressin would be expected to be elevated. The significance of this finding is unknown. The recent VASST trial, a randomized, controlled trial comparing norepinephrine alone to norepinephrine plus vasopressin at .03 units per minute showed no difference in outcome in the intent to treat population. An a priori defined subgroup analysis showed that the survival of patients receiving less than $15 \mu \mathrm{g} / \mathrm{min}$ norepinephrine at the time of randomization was better with vasopressin. It should be noted however that the pre-trial rationale for this stratification was based on exploring potential benefit in the $15 \mu \mathrm{g}$ or greater norepinephrine requirement population. Higher doses of vasopressin have been associated with cardiac, digital, and splanchnic ischemia and should be reserved for situations where alternative vasopressors have failed [96]. Cardiac output measurement to allow maintenance of a normal or elevated flow is desirable when these pure vasopressors are instituted.

5. We recommend that low dose dopamine not be used for renal protection (Grade 1A).

Rationale. A large randomized trial and meta-analysis comparing low-dose dopamine to placebo found no difference in either primary outcomes (peak serum creatinine, need for renal replacement, urine output, time to recovery of normal renal function), or secondary outcomes (survival to either ICU or hospital discharge, ICU stay, hospital stay, arrhythmias) [97, 98]. Thus the available data do not support administration of low doses of dopamine solely to maintain renal function.

6. We recommend that all patients requiring vasopressors have an arterial line placed as soon as practical if resources are available (Grade 1D).

Rationale. In shock states, estimation of blood pressure using a cuff is commonly inaccurate; use of an arterial cannula provides a more appropriate and reproducible measurement of arterial pressure. These catheters also allow continuous analysis so that decisions regarding therapy can be based on immediate and reproducible blood pressure information.

\section{G. Inotropic Therapy}

1. We recommend a dobutamine infusion be administered in the presence of myocardial dysfunction as suggested by elevated cardiac filling pressures and low cardiac output (Grade 1C).

2. We recommend against the use of a strategy to increase cardiac index to predetermined supranormal levels (Grade 1B).

Rationale. Dobutamine is the first-choice inotrope for patients with measured or suspected low cardiac output in the presence of adequate left ventricular filling pressure (or clinical assessment of adequate fluid resuscitation) and adequate mean arterial pressure. Septic patients who remain hypotensive after fluid resuscitation may have low, normal, or increased cardiac outputs. Therefore, treatment with a combined inotrope/vasopressor such as norepinephrine or dopamine is recommended if cardiac output is not measured. When the capability exists for monitoring cardiac output in addition to blood pressure, a vasopressor such as norepinephrine may be used separately to target specific levels of mean arterial pressure and cardiac output. Two large prospective clinical trials that included critically ill ICU patients who had severe sepsis failed to demonstrate benefit from increasing oxygen delivery to supranormal targets by use of dobutamine [99, 100]. These studies did not target specifically patients with severe sepsis and did not target the first 6 hours of resuscitation. The first 6 hours of resuscitation of sepsis induced hypoperfusion need to be treated separately from the later stages of severe sepsis (see initial resuscitation recommendations).

\section{H. Corticosteroids}

1. We suggest intravenous hydrocortisone be given only to adult septic shock patients after blood pressure is 
identified to be poorly responsive to fluid resuscitation and vasopressor therapy (Grade $2 \mathrm{C}$ ).

Rationale. One french multi-center, randomized, controlled trial (RCT) of patients in vasopressor-unresponsive septic shock (hypotension despite fluid resuscitation and vasopressors) showed a significant shock reversal and reduction of mortality rate in patients with relative adrenal insufficiency (defined as post-adrenocorticotropic hormone (ACTH) cortisol increase $9 \mu \mathrm{g} / \mathrm{dL}$ or less) [101]. Two additional smaller RCTs also showed significant effects on shock reversal with steroid therapy [102, 103]. However, a recent large, European multicenter trial (CORTICUS), which has been presented in abstract form but not yet published, failed to show a mortality benefit with steroid therapy of septic shock [104]. CORTICUS did show a faster resolution of septic shock in patients who received steroids. The use of the ACTH test (responders and nonresponders) did not predict the faster resolution of shock. Importantly, unlike the French trial, which only enrolled shock patients with blood pressure unresponsive to vasopressor therapy, the CORTICUS study included patients with septic shock, regardless of how the blood pressure responded to vasopressors. Although corticosteroids do appear to promote shock reversal, the lack of a clear improvement in mortality-coupled with known side effects of steroids such as increased risk of infection and myopathy-generally tempered enthusiasm for their broad use. Thus, there was broad agreement that the recommendation should be downgraded from the previous guidelines (Appendix B). There was considerable discussion and consideration by the committee on the option of encouraging use in those patients whose blood pressure was unresponsive to fluids and vasopressors, while strongly discouraging use in subjects whose shock responded well to fluids and pressors. However, this more complex set of recommendations was rejected in favor of the above single recommendation (see Appendix B).

2. We suggest the ACTH stimulation test not be used to identify the subset of adults with septic shock who should receive hydrocortisone (Grade 2B).

Rationale. Although one study suggested those who did not respond to ACTH with a brisk surge in cortisol (failure to achieve or $>9 \mu \mathrm{g} / \mathrm{dL}$ increase in cortisol 30-60 mins post-ACTH administration) were more likely to benefit from steroids than those who did respond, the overall trial population appeared to benefit regardless of ACTH result, and the observation of a potential interaction between steroid use and ACTH test was not statistically significant [101]. Furthermore, there was no evidence of this distinction between responders and nonresponders in a recent multicenter trial [104]. Commonly used cortisol immunoassays measure total cortisol (protein-bound and free) while free cortisol is the pertinent measurement.
The relationship between free and total cortisol varies with serum protein concentration. When compared to a reference method (mass spectrometry), cortisol immunoassays may over- or underestimate the actual cortisol level, affecting the assignment of patients to responders or nonresponders [105]. Although the clinical significance is not clear, it is now recognized that etomidate, when used for induction for intubation, will suppress the HPA axis [106].

3. We suggest that patients with septic shock should not receive dexamethasone if hydrocortisone is available (Grade 2B).

Rationale. Although often proposed for use until an ACTH stimulation test can be administered, we no longer suggest an ACTH test in this clinical situation (see \#3 above). Furthermore, dexamethasone can lead to immediate and prolonged suppression of the HPA axis after administration [107].

4. We suggest the daily addition of oral fludrocortisone $(50 \mu \mathrm{g})$ if hydrocortisone is not available and the steroid that is substituted has no significant mineralocorticoid activity. Fludrocortisone is considered optional if hydrocortisone is used (Grade 2C).

Rationale. One study added $50 \mu \mathrm{g}$ of fludrocortisone orally [101]. Since hydrocortisone has intrinsic mineralcorticoid activity, there is controversy as to whether fludrocortisone should be added.

5. We suggest clinicians wean the patient from steroid therapy when vasopressors are no longer required (Grade 2D).

Rationale. There has been no comparative study between a fixed duration and clinically guided regimen, or between tapering and abrupt cessation of steroids. Three RCTs used a fixed duration protocol for treatment [101, 103, 104], and in two RCTs, therapy was decreased after shock resolution [102, 108]. In four RCTs steroids were tapered over several days [102-104, 108], and in two RCTs [101, 109] steroids were withdrawn abruptly. One cross-over study showed hemodynamic and immunologic rebound effects after abrupt cessation of corticosteroids [110]. It remains uncertain whether outcome is affected by tapering of steroids or not.

6. We recommend doses of corticosteroids comparable to $>300 \mathrm{mg}$ hydrocortisone daily not be used in severe sepsis or septic shock for the purpose of treating septic shock (Grade 1A).

Rationale. Two randomized prospective clinical trials and a meta-analyses concluded that for therapy of severe sepsis 
or septic shock, high-dose corticosteroid therapy is ineffective or harmful [111-113]. Reasons to maintain higher doses of corticosteroid for medical conditions other than septic shock may exist.

7. We recommend corticosteroids not be administered for the treatment of sepsis in the absence of shock. There is, however, no contraindication to continuing maintenance steroid therapy or to using stress does steroids if the patient's endocrine or corticosteroid administration history warrants (Grade 1D).

Rationale. No studies exist that specifically target severe sepsis in the absence of shock that offer support for use of stress doses of steroids in this patient population. Steroids may be indicated in the presence of a prior history of steroid therapy or adrenal dysfunction. A recent preliminary study of stress dose level steroids in community- acquired pneumonia is encouraging but needs confirmation [114].

\section{Recombinant Human Activated Protein C (rhAPC)}

1. We suggest that adult patients with sepsis induced organ dysfunction associated with a clinical assessment of high risk of death, most of whom will have APACHE II $\geq 25$ or multiple organ failure, receive rhAPC if there are no contraindications (Grade 2B except for patients within 30 days of surgery where it is Grade 2C). Relative contraindications should also be considered in decision making.

2. We recommend that adult patients with severe sepsis and low risk of death, most of whom will have APACHE II $<20$ or one organ failure, do not receive rhAPC (Grade 1A).

Rationale. The evidence concerning use of rhAPC in adults is primarily based on two randomized controlled trials (RCTs): PROWESS (1,690 adult patients, stopped early for efficacy) [115] and ADDRESS (stopped early for futility) [116]. Additional safety information comes from an open-label observational study ENHANCE [117]. The ENHANCE trial also suggested early administration of rhAPC was associated with better outcomes.

PROWESS involved 1,690 patients and documented $6.1 \%$ in absolute total mortality reduction with a relative risk reduction (RRR) of $19.4 \%$, 95\% CI 6.6-30.5\%, number needed to treat (NNT):16 [115]. Controversy associated with the results focused on a number of subgroup analyses. Subgroup analyses have the potential to mislead due to the absence of an intent to treat, sampling bias, and selection error [118]. The analyses suggested increasing absolute and relative risk reduction with greater risk of death using both higher APACHE II scores and greater number of organ failures [119]. This led to drug approval for patients with high risk of death (such as
APACHE II $\geq 25$ ) and more than one organ failure in Europe.

The ADDRESS trial involved 2,613 patients judged to have a low risk of death at the time of enrollment. 28 day mortality from all causes was $17 \%$ on placebo vs. $18.5 \%$ on APC, relative risk (RR) 1.08, 95\% CI 0.92-1.28 [116]. Again, debate focused on subgroup analyses; analyses restricted to small subgroups of patients with APACHE II score over 25 , or more than one organ failures which failed to show benefit; however these patient groups also had a lower mortality than in PROWESS.

Relative risk reduction of death was numerically lower in the subgroup of patients with recent surgery $(n=502)$ in the PROWESS trial (30.7\% placebo vs. 27.8\% APC) [119] when compared to the overall study population (30.8\% placebo vs. 24.7\% APC) [115]. In the ADDRESS trial, patients with recent surgery and single organ dysfunction who received APC had significantly higher 28 day mortality rates $(20.7 \%$ vs. $14.1 \%, \mathrm{p}=0.03, \mathrm{n}=635)$ [116].

Serious adverse events did not differ in the studies [115-117] with the exception of serious bleeding, which occurred more often in the patients treated with APC: $2 \%$ vs. $3.5 \%$ (PROWESS; $p=0.06$ ) [115]; $2.2 \%$ vs. $3.9 \%$ (ADDRESS; $p<0.01$ ) [116]; 6.5\% (ENHANCE, open label) [117]. The pediatric trial and implications are discussed in the pediatric consideration section of this manuscript (see Appendix $\mathrm{C}$ for absolute contraindications to use of rhAPC and prescribing information for relative contraindications).

Intracranial hemorrhage (ICH) occurred in the PROWESS trial in $0.1 \%$ (placebo) and $0.2 \%$ (APC) (n.s.) [106], in the ADDRESS trial $0.4 \%$ (placebo) vs. $0.5 \%$ (APC) (n. s.) [116]; in ENHANCE 1.5\% [108]. Registry studies of rhAPC report higher bleeding rates than randomized controlled trials, suggesting that the risk of bleeding in actual practice may be greater than reported in PROWESS and ADDRESS [120, 121].

The two RCTs in adult patients were methodologically strong, precise, and provide direct evidence regarding death rates. The conclusions are limited, however, by inconsistency that is not adequately resolved by subgroup analyses (thus the designation of moderate quality evidence). Results, however, consistently fail to show benefit for the subgroup of patients at lower risk of death, and consistently show increases in serious bleeding. The RCT in pediatric severe sepsis failed to show benefit and has no important limitations. Thus, for low risk and pediatric patients, we rate the evidence as high quality.

For adult use there is probable mortality reduction in patients with clinical assessment of high risk of death, most of whom will have APACHE II $\geq 25$ or multiple organ failure. There is likely no benefit in patients with low risk of death, most of whom will have APACHE II $<20$ or single organ dysfunction. The effects in patients with more than one organ failure but APACHE II $<25$ are unclear and in that circumstance one may use clinical assessment of the 
risk of death and number of organ failures to support decision. There is a certain increased risk of bleeding with administration of rhAPC which may be higher in surgical patients and in the context of invasive procedures. Decision on utilization depends upon assessing likelihood of mortality reduction versus increases in bleeding and cost (see appendix D for nominal committee vote on recommendation for rhAPC). A European Regulatory mandated randomized controlled trial of rhAPC vs. placebo in patients with septic shock is now ongoing [122].

\section{J. Blood Product Administration}

1. Once tissue hypoperfusion has resolved and in the absence of extenuating circumstances, such as myocardial ischemia, severe hypoxemia, acute hemorrhage, cyanotic heart disease, or lactic acidosis (see recommendations for initial resuscitation), we recommend that red blood cell transfusion occur when hemoglobin decreases to $<7.0 \mathrm{~g} / \mathrm{dL}(<70 \mathrm{~g} / \mathrm{L})$ to target a hemoglobin of 7.0-9.0 g/dL $(70-90 \mathrm{~g} / \mathrm{L})$ in adults (Grade 1B).

Rationale. Although the optimum hemoglobin for patients with severe sepsis has not been specifically investigated, the Transfusion Requirements in Critical Care trial suggested that a hemoglobin of $7-9 \mathrm{~g} / \mathrm{dL}(70-90 \mathrm{~g} / \mathrm{L})$ when compared to $10-12 \mathrm{~g} / \mathrm{dL}(100-200 \mathrm{~g} / \mathrm{L})$ was not associated with increased mortality rate in adults [123]. Red blood cell transfusion in septic patients increases oxygen delivery but does not usually increase oxygen consumption [124-126]. This transfusion threshold of $7 \mathrm{~g} / \mathrm{dL}(70 \mathrm{~g} / \mathrm{L})$ contrasts with the early goal-directed resuscitation protocol that uses a target hematocrit of $30 \%$ in patients with low $\mathrm{S}_{\mathrm{CV}} \mathrm{O}_{2}$ (measured in superior vena cava) during the first $6 \mathrm{hrs}$ of resuscitation of septic shock.

2. We recommend that erythropoietin not be used as a specific treatment of anemia associated with severe sepsis, but may be used when septic patients have other accepted reasons for administration of erythropoietin such as renal failure-induced compromise of red blood cell production (Grade 1B).

Rationale. No specific information regarding erythropoietin use in septic patients is available, but clinical trials in critically ill patients show some decrease in red cell transfusion requirement with no effect on clinical outcome $[127,128]$. The effect of erythropoietin in severe sepsis and septic shock would not be expected to be more beneficial than in other critical conditions. Patients with severe sepsis and septic shock may have coexisting conditions that do warrant use of erythropoietin.

3 . We suggest that fresh frozen plasma not be used to correct laboratory clotting abnormalities in the absence of bleeding or planned invasive procedures (Grade 2D).
Rationale. Although clinical studies have not assessed the impact of transfusion of fresh frozen plasma on outcomes in critically ill patients, professional organizations have recommended fresh frozen plasma for coagulopathy when there is a documented deficiency of coagulation factors (increased prothrombin time, international normalized ratio, or partial thromboplastin time) and the presence of active bleeding or before surgical or invasive procedures [129-131]. In addition, transfusion of fresh frozen plasma in nonbleeding patients with mild abnormalities of prothrombin time usually fails to correct the prothrombin time [132]. There are no studies to suggest that correction of more severe coagulation abnormalities benefits patients who are not bleeding.

4. We recommend against antithrombin administration for the treatment of severe sepsis and septic shock (Grade 1B).

Rationale. A phase III clinical trial of high-dose antithrombin did not demonstrate any beneficial effect on 28-day all-cause mortality in adults with severe sepsis and septic shock. High-dose antithrombin was associated with an increased risk of bleeding when administered with heparin [133]. Although a post hoc subgroup analysis of patients with severe sepsis and high risk of death showed better survival in patients receiving antithrombin, antithrombin cannot be recommended at this time until further clinical trials are performed [134].

5. In patients with severe sepsis, we suggest that platelets should be administered when counts are $<5000 / \mathrm{mm}^{3}$ $\left(5 \times 10^{9} / \mathrm{L}\right)$ regardless of apparent bleeding. Platelet transfusion may be considered when counts are $5,000-30,000 / \mathrm{mm}^{3}\left(5-30 \times 10^{9} / \mathrm{L}\right)$ and there is a significant risk of bleeding. Higher platelet counts $\left(\geq 50,000 / \mathrm{mm}^{3}\left(50 \times 10^{9} / \mathrm{L}\right)\right)$ are typically required for surgery or invasive procedures (Grade $2 \mathrm{D}$ ).

Rationale. Guidelines for transfusion of platelets are derived from consensus opinion and experience in patients undergoing chemotherapy. Recommendations take into account the etiology of thrombocytopenia, platelet dysfunction, risk of bleeding, and presence of concomitant disorders $[129,131]$.

\section{Supportive Therapy of Severe Sepsis}

\section{A. Mechanical Ventilation of Sepsis-Induced Acute Lung Injury (ALI)/Acute Respiratory Distress Syndrome $(A R D S)$}

1. We recommend that clinicians target a tidal volume of $6 \mathrm{ml} / \mathrm{kg}$ (predicted) body weight in patients with ALI/ARDS (Grade 1B). 
2. We recommend that plateau pressures be measured in patients with ALI/ARDS and that the initial upper limit goal for plateau pressures in a passively inflated patient be $\leq 30 \mathrm{~cm} \mathrm{H}_{2} \mathrm{O}$. Chest wall compliance should be considered in the assessment of plateau pressure (Grade 1C).

Rationale. Over the past $10 \mathrm{yrs}$, several multi-center randomized trials have been performed to evaluate the effects of limiting inspiratory pressure through moderation of tidal volume [135-139]. These studies showed differing results that may have been caused by differences between airway pressures in the treatment and control groups $[135,140]$. The largest trial of a volumeand pressure-limited strategy showed a 9\% decrease of all-cause mortality in patients with ALI or ARDS ventilated with tidal volumes of $6 \mathrm{~mL} / \mathrm{kg}$ of predicted body weight (PBW), as opposed to $12 \mathrm{~mL} / \mathrm{kg}$, and aiming for a plateau pressure $\leq 30 \mathrm{~cm} \mathrm{H}_{2} \mathrm{O}$ [135]. The use of lung protective strategies for patients with ALI is supported by clinical trials and has been widely accepted, but the precise choice of tidal volume for an individual patient with ALI may require adjustment for such factors as the plateau pressure achieved, the level of PEEP chosen, the compliance of the thoracoabdominal compartment and the vigor of the patient's breathing effort. Some clinicians believe it may be safe to ventilate with tidal volumes higher than $6 \mathrm{ml} / \mathrm{kg} \mathrm{PBW}$ as long as the plateau pressure can be maintained $\leq 30 \mathrm{~cm} \mathrm{H}_{2} \mathrm{O}$ [141, 142]. The validity of this ceiling value will depend on breathing effort, as those who are actively inspiring generate higher trans-alveolar pressures for a given plateau pressure than those who are passively inflated. Conversely, patients with very stiff chest walls may require plateau pressures higher than $30 \mathrm{~cm} \mathrm{H}_{2} \mathrm{O}$ to meet vital clinical objectives. One retrospective study suggested that tidal volumes should be lowered even with plateau pressures that are $\leq 30 \mathrm{~cm} \mathrm{H}_{2} \mathrm{O}$ [143]. An additional observational study suggested that knowledge of the plateau pressures was associated with lower plateau pressures; however in this trial, plateau pressure was not independently associated with mortality rates across a wide range of plateau pressures that bracketed $30 \mathrm{~cm} \mathrm{H}_{2} \mathrm{O}$ [144]. The largest clinical trial employing a lung protective strategy coupled limited pressure with limited tidal volumes to demonstrate a mortality benefit [135].

High tidal volumes that are coupled with high plateau pressures should be avoided in ALI/ARDS. Clinicians should use as a starting point the objective of reducing tidal volumes over $1-2 \mathrm{hrs}$ from its initial value toward the goal of a "low" tidal volume $(\approx 6 \mathrm{~mL}$ per kilogram of predicted body weight) achieved in conjunction with an end-inspiratory plateau pressure $\leq 30 \mathrm{~cm} \mathrm{H}_{2} \mathrm{O}$. If plateau pressure remains $>30$ after reduction of tidal volume to $6 \mathrm{ml} / \mathrm{kg} / \mathrm{PBW}$, tidal volume should be reduced further to as low as $4 \mathrm{ml} / \mathrm{kg} / \mathrm{PBW}$ (see Appendix E for ARDSnet ventilator management and formula to calculate predicted body weight).

No single mode of ventilation (pressure control, volume control, airway pressure release ventilation, high frequency ventilation, etc.) has been consistently shown advantageous when compared with any other that respects the same principles of lung protection.

3. We recommend that hypercapnia (allowing $\mathrm{PaCO}_{2}$ to increase above its pre-morbid baseline, so-called permissive hypercapnia) be allowed in patients with ALI/ARDS if needed to minimize plateau pressures and tidal volumes (Grade 1C).

Rationale. An acutely elevated $\mathrm{PaCO}_{2}$ may have physiologic consequences that include vasodilation as well as an increased heart rate, blood pressure, and cardiac output. Allowing modest hypercapnia in conjunction with limiting tidal volume and minute ventilation has been demonstrated to be safe in small, nonrandomized series $[145,146]$. Patients treated in larger trials that have the goal of limiting tidal volumes and airway pressures have demonstrated improved outcomes, but permissive hypercapnia was not a primary treatment goal in these studies [135]. The use of hypercapnia is limited in patients with preexisting metabolic acidosis and is contraindicated in patients with increased intracranial pressure. Sodium bicarbonate or tromethamine $\left(\mathrm{THAM}^{\circledR}\right)$ infusion may be considered in selected patients to facilitate use of permissive hypercarbia [147, 148].

4. We recommend that positive end-expiratory pressure (PEEP) be set so as to avoid extensive lung collapse at end-expiration (Grade 1C).

Rationale. Raising PEEP in ALI/ARDS keeps lung units open to participate in gas exchange. This will increase $\mathrm{PaO}_{2}$ when PEEP is applied through either an endotracheal tube or a face mask [149-151]. In animal experiments, avoidance of end-expiratory alveolar collapse helps minimize ventilator induced lung injury (VILI) when relatively high plateau pressures are in use. One large multi-center trial of the protocol-driven use of higher PEEP in conjunction with low tidal volumes did not show benefit or harm when compared to lower PEEP levels [152]. Neither the control nor experimental group in that study, however, was clearly exposed to hazardous plateau pressures. A recent multi-center Spanish trial compared a high PEEP, low-moderate tidal volume approach to one that used conventional tidal volumes and the least PEEP achieving adequate oxygenation. A marked survival advantage favored the former approach in high acuity patients with ARDS [153]. Two options are recommended for PEEP titration. One option is to titrate PEEP (and tidal volume) according to bedside measurements of thoracopulmonary compliance with the objective of obtaining 
the best compliance, reflecting a favorable balance of lung recruitment and overdistention [154]. The second option is to titrate PEEP based on severity of oxygenation deficit and guided by the $\mathrm{F}_{\mathrm{I}} \mathrm{O}_{2}$ required to maintain adequate oxygenation [135] (see Appendix D.). Whichever the indicator-compliance or oxygenation-recruiting maneuvers are reasonable to employ in the process of PEEP selection. Blood pressure and oxygenation should be monitored and recruitment discontinued if deterioration in these parameters is observed. A PEEP $>5 \mathrm{~cm} \mathrm{H}_{2} \mathrm{O}$ is usually required to avoid lung collapse [155].

5. We suggest prone positioning in ARDS patients requiring potentially injurious levels of $\mathrm{F}_{\mathrm{I}} \mathrm{O}_{2}$ or plateau pressure who are not at high risk for adverse consequences of positional changes in those facilities who have experience with such practices (Grade 2C).

Rationale. Several smaller studies and one larger study have shown that a majority of patients with ALI/ARDS respond to the prone position with improved oxygenation [156-159]. One large multi-center trial of prone positioning for approximately $7 \mathrm{hrs} /$ day did not show improvement in mortality rates in patients with ALI/ARDS; however, a post hoc analysis suggested improvement in those patients with the most severe hypoxemia by $\mathrm{PaO}_{2} / \mathrm{F}_{\mathrm{I}} \mathrm{O}_{2}$ ratio, in those exposed to high tidal volumes, and those who improved $\mathrm{CO}_{2}$ exchange as a result of proning [159]. A second large trial of prone positioning, conducted for an average of approximately 8 hours per day for 4 days in adults with hypoxemic respiratory failure of low-moderate acuity, confirmed improvement in oxygenation but also failed to show a survival advantage [160]. However, a randomized study that extended the length of time for proning each day to a mean of 17 hours for a mean of 10 days supported benefit of proning, with randomization to supine position an independent risk factor for mortality by multivariate analysis [161]. Prone positioning may be associated with potentially life-threatening complications, including accidental dislodgment of the endotracheal tube and central venous catheters, but these complications can usually be avoided with proper precautions.

6. A) Unless contraindicated, we recommend mechanically ventilated patients be maintained with the head of the bed elevated to limit aspiration risk and to prevent the development of ventilator-associated pneumonia (Grade 1B).

B) We suggest that the head of bed is elevated approximately 30-45 degrees (Grade 2C).

Rationale. The semirecumbent position has been demonstrated to decrease the incidence of ventilator-associated pneumonia (VAP) [164]. Enteral feeding increased the risk of developing VAP; $50 \%$ of the patients who were fed en- terally in the supine position developing VAP [162]. However, the bed position was only monitored once a day, and patients who did not achieve the desired bed elevation were not included in the analysis [162]. A recent study did not show a difference in in incidence of VAP between patients maintained in supine and semirecumbent positions [163]. In this study, patients in the semirecumbent position did not consistently achieve the desired head of the bed elevation, and the head of bed elevation in the supine group approached that of the semirecumbent group by day 7 [163]. When necessary, patients may be laid flat for procedures, hemodynamic measurements, and during episodes of hypotension. Patients should not be fed enterally with the head of the bed at $0^{\circ}$.

7. We suggest that noninvasive mask ventilation (NIV) only be considered in that minority of ALI/ARDS patients with mild-moderate hypoxemic respiratory failure (responsive to relatively low levels of pressure support and PEEP) with stable hemodynamics who can be made comfortable and easily arousable, who are able to protect the airway, spontaneously clear the airway of secretions, and are anticipated to recover rapidly from the precipitating insult. A low threshold for airway intubation should be maintained (Grade 2B).

Rationale. Obviating the need for airway intubation confers multiple advantages: better communication, lower incidence of infection, reduced requirements for sedation. Two RCTs demonstrate improved outcome with the use of NIV when it can be employed successfully $[164,165]$. Unfortunately, only a small percentage of patients with life threatening hypoxemia can be managed in this way.

8. We recommend that a weaning protocol be in place, and mechanically ventilated patients with severe sepsis undergo spontaneous breathing trials on a regular basis to evaluate the ability to discontinue mechanical ventilation when they satisfy the following criteria: a) arousable; b) hemodynamically stable (without vasopressor agents); c) no new potentially serious conditions; d) low ventilatory and end-expiratory pressure requirements; and e) $\mathrm{F}_{\mathrm{I}} \mathrm{O}_{2}$ requirements that could be safely delivered with a face mask or nasal cannula. If the spontaneous breathing trial is successful, consideration should be given for extubation (see Appendix E). Spontaneous breathing trial options include a low level of pressure support, continuous positive airway pressure $\left(\approx 5 \mathrm{~cm} \mathrm{H}_{2} \mathrm{O}\right)$ or a T-piece (Grade $\left.1 \mathrm{~A}\right)$.

Rationale. Recent studies demonstrate that daily spontaneous breathing trials in appropriately selected patients reduce the duration of mechanical ventilation [166-169]. Successful completion of spontaneous breathing trials leads to a high likelihood of successful discontinuation of mechanical ventilation. 
9. We recommend against the routine use of the pulmonary artery catheter for patients with ALI/ARDS (Grade 1A).

Rationale. While insertion of a pulmonary artery catheter may provide useful information on a patient's volume status and cardiac function, potential benefits of such information may be confounded by differences in interpretation of results [170-172], lack of correlation of pulmonary artery occlusion pressures with clinical response [173], and absence of a proven strategy to use catheter results to improve patient outcomes [174]. Two multi-center randomized trials: one in patients with shock or acute lung injury [175], and one in patients with acute lung injury [176] failed to show benefit with the routine use of pulmonary artery catheters in patients with acute lung injury. In addition, other studies in different types of critically ill patients have failed to show definitive benefit with routine use of the pulmonary artery catheter [177-179]. Well-selected patients remain appropriate candidates for pulmonary artery catheter insertion when the answers to important management decisions depend on information only obtainable from direct measurements made within the pulmonary artery.

10. To decrease days of mechanical ventilation and ICU length of stay we recommend a conservative fluid strategy for patients with established acute lung injury who do not have evidence of tissue hypoperfusion (Grade 1C).

Rationale. Mechanisms for the development of pulmonary edema in patients with acute lung injury include increased capillary permeability, increased hydrostatic pressure and decreased oncotic pressure $[180,181]$. Small prospective studies in patients with critical illness and acute lung injury have suggested that less weight gain is associated with improved oxygenation [182] and fewer days of mechanical ventilation $[183,184]$. Use of a fluid conservative strategy directed at minimizing fluid infusion and weight gain in patients with acute lung injury based on either a central venous catheter or a pulmonary artery catheter along with clinical parameters to guide treatment strategies led to fewer days of mechanical ventilation and reduced length of ICU stay without altering the incidence of renal failure or mortality rates [185]. Of note, this strategy was only used in patients with established acute lung injury, some of whom had shock present. Active attempts to reduce fluid volume were conducted only during periods free of shock.

\section{B. Sedation, Analgesia, and Neuromuscular Blockade in Sepsis}

1. We recommend sedation protocols with a sedation goal when sedation of critically ill mechanically ventilated patients with sepsis is required (Grade 1B).
Rationale. A growing body of evidence indicates that the use of protocols for sedation of critically ill ventilated patients can reduce the duration of mechanical ventilation and ICU and hospital length of stay [186-188]. A randomized, controlled clinical trial found that protocol use resulted in reduced duration of mechanical ventilation, reduced lengths of stay, and reduced tracheostomy rates [186].

A report describing the implementation of protocols, including sedation and analgesia, using a short-cycle improvement methodology in the management of critically ill patients demonstrated a decrease in the cost per patient day and a decrease of ICU length of stay [187]. Furthermore, a prospective before-and-after study on the implementation of a sedation protocol demonstrated enhanced quality of sedation with reduced drug costs. Although this protocol also may have contributed to a longer duration of mechanical ventilation, ICU discharge was not delayed [188]. Despite the lack of evidence regarding the use of subjective methods of evaluation of sedation in septic patients, the use of a sedation goal has been shown to decrease the duration of mechanical ventilation in critically ill patients [186]. Several subjective sedation scales have been described in the medical literature. Currently, however, there is not a clearly superior sedation evaluation methodology against which these sedation scales can be evaluated [189]. The benefits of sedation protocols appear to outweigh the risks.

2. We recommend intermittent bolus sedation or continuous infusion sedation to predetermined end points (e.g., sedation scales) with daily interruption/lightening of continuous infusion sedation with awakening and retitration if necessary for sedation administration to septic mechanically ventilated patients (Grade 1B).

Rationale. Although not specifically studied in patients with sepsis, the administration of intermittent sedation, daily interruption, and retitration or systemic titration to a predefined end point have been demonstrated to decrease the duration of mechanical ventilation $[186,189,190]$. Patients receiving neuromuscular blocking agents (NMBAs) must be individually assessed regarding discontinuation of sedative drugs because neuromuscular blocking drugs must also be discontinued in that situation. The use of intermittent vs. continuous methods for the delivery of sedation in critically ill patients has been examined. An observational study of mechanically-ventilated patients showed that patients receiving continuous sedation had significantly longer durations of mechanical ventilation and ICU and hospital length of stay [191].

Similarly, a prospective, controlled study in 128 mechanically-ventilated adults receiving continuous intravenous sedation demonstrated that a daily interruption in the "continuous" sedative infusion until the patient was 
awake decreased the duration of mechanical ventilation and ICU length of stay [192]. Although the patients did receive continuous sedative infusions in this study, the daily interruption and awakening allowed for titration of sedation, in effect, making the dosing intermittent. Systematic (protocolized) titration to a predefined end point has also been shown to alter outcome [186]. Additionally, a randomized prospective blinded observational study demonstrated that although myocardial ischemia is common in critically ill ventilated patients, daily sedative interruption is not associated with an increased occurrence of myocardial ischemia [193]. Thus, the benefits of daily interruption of sedation appear to outweigh the risks. These benefits include potentially shorter duration of mechanical ventilation and ICU stay, better assessment of neurologic function, and reduced costs.

3. We recommend that NMBAs be avoided if possible in the septic patient due to the risk of prolonged neuromuscular blockade following discontinuation. If NMBAs must be maintained, either intermittent bolus as required or continuous infusion with monitoring the depth of blockade with train-of-four monitoring should be used (Grade 1B).

Rationale. Although NMBAs are often administered to critically ill patients, their role in the ICU setting is not well defined. No evidence exists that maintaining neuromuscular blockade in this patient population reduces mortality or major morbidity. In addition, no studies have been published that specifically address the use of NMBAs in septic patients.

The most common indication for NMBA use in the ICU is to facilitate mechanical ventilation [194]. When appropriately utilized, NMBAs may improve chest wall compliance, prevent respiratory dyssynchrony, and reduce peak airway pressures [195]. Muscle paralysis may also reduce oxygen consumption by decreasing the work of breathing and respiratory muscle blood flow [196]. However, a randomized, placebo-controlled clinical trial in patients with severe sepsis demonstrated that oxygen delivery, oxygen consumption, and gastric intramucosal $\mathrm{pH}$ were not improved during profound neuromuscular blockade [197].

An association between NMBA use and myopathies and neuropathies has been suggested by case studies and prospective observational studies in the critical care population [195, 198-201]. The mechanisms by which NMBA's produced or contribute to myopathies and neuropathies in critically ill patients are presently unknown. There appears to be an added association with the concurrent use o NMBA's and steroids. Although no specific studies exist specific to the septic patient population, it seems clinically prudent based on existent knowledge that NMBA's not be administered unless there is a clear indication for neuromuscular blockade that can not be safely achieved with appropriate sedation and analgesia" [195].

Only one prospective, randomized clinical trial has evaluated peripheral nerve stimulation vs. standard clinical assessment in ICU patients. Rudis et al. [202] randomized 77 critically ill patients requiring neuromuscular blockade in the ICU to receive dosing of vecuronium based on train-of-four stimulation or clinical assessment (control). The peripheral nerve stimulation group received less drug and recovered neuromuscular function and spontaneous ventilation faster than the control group. Nonrandomized observational studies have suggested that peripheral nerve monitoring reduces or has no effect on clinical recovery from NMBAs in the ICU setting [203, 204].

Benefits to neuromuscular monitoring, including faster recovery of neuromuscular function and, shorter intubation times, appear to exist. A potential for cost savings (reduced total dose of NMBAs and shorter intubation times) also may exist, although this has not been studied formally.

\section{Glucose Control}

1. We recommend that, following initial stabilization, patients with severe sepsis and hyperglycemia who are admitted to the ICU receive IV insulin therapy to reduce blood glucose levels (Grade 1B).

2. We suggest use of a validated protocol for insulin dose adjustments and targeting glucose levels to the $<150 \mathrm{mg} / \mathrm{dl}$ range (Grade 2C).

3 . We recommend that all patients receiving intravenous insulin receive a glucose calorie source and that blood glucose values be monitored every 1-2 hours until glucose values and insulin infusion rates are stable and then every 4 hours thereafter (Grade 1C).

4. We recommend that low glucose levels obtained with point-of-care testing of capillary blood be interpreted with caution, as such measurements may overestimate arterial blood or plasma glucose values (Grade 1B).

Rationale. The consensus on glucose control in severe sepsis was achieved at the first committee meeting and subsequently approved by the entire committee (see Appendix $\mathrm{G}$ for committee vote). One large randomized single center trial in a predominantly cardiac surgical ICU demonstrated a reduction in ICU mortality with intensive IV insulin (Leuven Protocol) targeting blood glucose to $80-110 \mathrm{mg} / \mathrm{dl}$ (for all patients relative $43 \%$, and absolute $3.4 \%$ mortality reduction, and for those with $>5$ day ICU length of stays (LOS) a $48 \%$ relative and $9.6 \%$ absolute mortality reduction) [205]. A reduction in organ dysfunction and ICU LOS (from a median of 15 to12 days) was also observed in the subset with ICU LOS $>5$ days. A second randomized trial of intensive insulin therapy using the Leuven Protocol enrolled medical ICU 
patients with an anticipated ICU LOS of $>3$ days in three MICUs [206]. Overall, mortality was not reduced but ICU and hospital LOS were reduced associated with earlier weaning from mechanical ventilation and less acute kidney injury. In patients with a medical ICU LOS $>3$ days, hospital mortality was reduced with intensive insulin therapy ( $43 \%$ versus $52.5 \% ; p=0.009$ ). However, investigators were unsuccessful in predicting ICU LOS and 433 patients $(36 \%)$ had an ICU LOS of $<3$ days. Furthermore, use of the Leuven Protocol in the medical ICU resulted in a nearly three-fold higher rate of hypoglycemia than in the original experience $(18 \%$ versus $6.2 \%$ of patients) $[205,206]$.

One large before-and-after observational trial showed a $29 \%$ relative and $6.1 \%$ absolute reduction in mortality and a $10.8 \%$ reduction in median ICU LOS [207]. In a subgroup of 53 patients with septic shock there was an absolute mortality reduction of $27 \%$ and a relative reduction of $45 \%(p=0.02)$. Two additional observational studies report an association of mean glucose levels with reductions in mortality, polyneuropathy, acute renal failure, nosocomial bacteremia, and number of transfusions, and suggest a glucose threshold for improved mortality lies somewhere between 145 and $180 \mathrm{mg} / \mathrm{dl}$ [208, 209]. However, a large observational study $(n=7,049)$ suggested that both a lower mean glucose and less variation of blood glucose may be important [210]. A meta-analysis of 35 trials on insulin therapy in critically ill patients, including 12 randomized trials, demonstrated a $15 \%$ reduction in short term mortality (RR $0.85,95 \%$ confidence interval $0.75-0.97$ ) but did not include any studies of insulin therapy in medical ICUs [211].

Two additional multicenter RCTs of intensive insulin therapy, one focusing on patients with severe sepsis (VISEP) and the second on medical and surgical ICU patients, failed to demonstrate improvement in mortality, but are not yet published [212, 213]. Both stopped earlier than planned because of high rates of hypoglycemia and adverse events in the intensive insulin groups. A large RCT that is planned to compare targeting $80-110 \mathrm{mg} / \mathrm{dl}$ $(4.5-6.0 \mathrm{mmol} / \mathrm{L})$ versus $140-180 \mathrm{mg} / \mathrm{dl}(8-10 \mathrm{mmol} / \mathrm{L})$ and recruit more than 6,000 patients (Normoglycemia in Intensive Care Evaluation and Survival Using Glucose Algorithm Regulation, or NICE-SUGAR) is ongoing [214].

Several factors may affect the accuracy and reproducibility of point-of-care testing of blood capillary blood glucose, including the type and model of the device used, user expertise, and patient factors including hematocrit (false elevation with anemia), $\mathrm{PaO}_{2}$, and drugs [215]. One report showed overestimation of arterial plasma glucose values by capillary point-of-care testing sufficient to result in different protocol-specified insulin dose titration. The disagreement between protocol-recommended insulin doses was largest when glucose values were low [216]. A recent review of 12 published insulin infusion protocols for critically ill patients showed wide variability in insulin dose recommendations and variable glucose control during simulation [217]. This lack of consensus about optimal dosing of IV insulin may reflect variability in patient factors (severity of illness, surgical vs. medical settings, etc) or practice patterns (e. g., approaches to feeding, IV dextrose) in the environments in which these protocols were developed and tested. Alternatively, some protocols may be more effective than other protocols. This conclusion is supported by the wide variability in hypoglycemia rates reported with protocols [205-207, 212, 213]. Thus, the use of a validated and safe intensive insulin protocol is important not only for clinical care but also for the conduct of clinical trials to avoid hypoglycemia, adverse events, and premature termination of these trials before the efficacy signal, if any, can be determined.

The finding of reduced morbidity and mortality within the longer ICU length of stay subsets along with acceptable cost weighed heavily on our recommendation to attempt glucose control after initial stabilization of the patient with hyperglycemia and severe sepsis. However, the mortality benefit and safety of intensive insulin therapy (goal to normalize blood glucose) has been questioned by 2 recent trials and we recommend maintaining glucose levels $<150 \mathrm{mg} / \mathrm{dl}$ until recent and ongoing trials are published or completed. Further study of protocols that have been validated to be safe and effective for controlling blood glucose concentrations and blood glucose variation in the severe sepsis population are needed.

\section{Renal Replacement}

1. We suggest that continuous renal replacement therapies and intermittent hemodialysis are equivalent in patients with severe sepsis and acute renal failure (Grade 2B).

2. We suggest the use of continuous therapies to facilitate management of fluid balance in hemodynamically unstable septic patients (Grade 2D).

Rationale. Although numerous nonrandomized studies have reported a nonsignificant trend toward improved survival using continuous methods [218-225], 2 metaanalyses [226, 227] report the absence of significant difference in hospital mortality between patients who receive continuous and intermittent renal replacement therapies. This absence of apparent benefit of one modality over the other persists even when the analysis is restricted to only randomized studies [227]. To date, 5 prospective randomized studies have been published [228-232]. Four of them found no significant difference in mortality [229-232]. One study found significantly higher mortality in the continuous treatment group [228], but imbalanced randomization had led to a higher baseline severity of illness in this group. When a multivariable model was used to adjust for severity of illness, no difference in mortality was 
apparent between the groups [228]. It is important to note that most studies comparing modes of renal replacement in the critically ill have included a small number of patients and some major weaknesses (randomization failure, modifications of therapeutic protocol during the study period, combination of different types of continuous renal replacement therapies, small number of heterogenous groups of patients enrolled). The most recent and largest randomized study [232] enrolled 360 patients and found no significant difference in survival between the 2 groups. Moreover, there is no current evidence to support the use of continuous therapies in sepsis independent of renal replacement needs.

Concerning the hemodynamic tolerance of each method, no current evidence exists to support a better tolerance with continuous treatments. Only 2 prospective studies [230, 233] have reported a better hemodynamic tolerance with continuous treatment, with no improvement in regional perfusion [233] and no survival benefit [230]. Four other prospective studies did not find any significant difference in mean arterial pressure or drop in systolic pressure between the 2 methods [229, 231, 232, 234]. Concerning fluid balance management, 2 studies report a significant improvement in goal achievement with continuous methods [228, 230]. In summary, current evidence is insufficient to draw strong conclusions regarding the mode of replacement therapy for acute renal failure in septic patients.

Four randomized, controlled trials have addressed whether the dose of continuous renal replacement affects outcomes in patients with acute renal failure [235-238]. Three found improved mortality in patients receiving higher doses of renal replacement [235, 237, 238], while one [236] did not. None of these trials was conducted specifically in patients with sepsis. Although the weight of current evidence suggests that higher doses of renal replacement may be associated with improved outcomes, these results may not be easily generalizable. The results of 2 very large multicenter randomized trials comparing the dose of renal replacement (ATN in the United States and RENAL in Australia and New Zealand) will be available in 2008 and will greatly inform practice.

\section{E. Bicarbonate Therapy}

1. We recommend against the use of sodium bicarbonate therapy for the purpose of improving hemodynamics or reducing vasopressor requirements in patients with hypoperfusion-induced lactic acidemia with $\mathrm{pH} \geq 7.15$ (Grade 1B).

Rationale. No evidence supports the use of bicarbonate therapy in the treatment of hypoperfusion-induced lactic acidemia associated with sepsis. Two randomized, blinded, crossover studies that compared equimolar saline and bicarbonate in patients with lactic acidosis failed to reveal any difference in hemodynamic variables or vasopressor requirements. [239, 240] The number of patients with $\mathrm{pH}<7.15$ in these studies was small. Bicarbonate administration has been associated with sodium and fluid overload, an increase in lactate and $\mathrm{pCO}_{2}$, and a decrease in serum ionized calcium; but the relevance of these parameters to outcome is uncertain. The effect of bicarbonate administration on hemodynamics and vasopressor requirements at lower $\mathrm{pH}$ as well as the effect on clinical outcomes at any $\mathrm{pH}$ is unknown. No studies have examined the effect of bicarbonate administration on outcomes.

\section{F. Deep Vein Thrombosis Prophylaxis}

1. We recommend that severe sepsis patients receive deep vein thrombosis (DVT) prophylaxis with either (a) low-dose unfractionated heparin (UFH) administered b.i.d. or t.i.d. or (b) daily low-molecular weight heparin (LMWH) unless there are contraindications (i. e., thrombocytopenia, severe coagulopathy, active bleeding, recent intracerebral hemorrhage) (Grade 1A).

2. We recommend that septic patients who have a contraindication for heparin use receive mechanical prophylactic device such as graduated compression stockings (GCS) or intermittent compression devices (ICD) unless contraindicated (Grade 1A).

3 . We suggest that in very high-risk patients such as those who have severe sepsis and history of DVT, trauma, or orthopedic surgery, a combination of pharmacologic and mechanical therapy be used unless contraindicated or not practical (Grade 2C).

4. We suggest that in patients at very high risk, LMWH be used rather than UFH as LMWH is proven superior in other high-risk patients (Grade 2C).

Rationale. ICU patients are at risk for DVT [241]. Significant evidence exists for benefit of DVT prophylaxis in ICU patients in general. No reasons suggest that severe sepsis patients would be different from the general patient population.

Nine randomized placebo controlled clinical trials of DVT prophylaxis in general populations of acutely ill patients exist [242-250]. All 9 trials showed reduction in DVT or PE. The prevalence of infection/sepsis was $17 \%$ in all studies in which this was ascertainable, with a $52 \%$ prevalence of infection/sepsis patients in the study that included ICU patients only. Benefit of DVT prophylaxis is also supported by meta-analyses [251, 252]. With that in mind, DVT prophylaxis would appear to have a high grade for quality of evidence (A). As the risk of administration to the patient is small, the gravity of the potential result of not administering is great, and the cost is low, the grading of the strength of the recommendation is strong. The evidence supports equivalency of LMWH and UFH in gen- 
eral medical populations. A recent meta-analysis comparing b.i.d. and t.i.d. UFH demonstrated that t.i.d. UFH produced better efficacy and b.i.d. less bleeding [253]. Practitioners should use underlying risk for VTE and bleeding to individualize choice of b.i.d. versus t.i.d.

The cost of LMWH is greater and the frequency of injection is less. UFH is preferred over LMWH in patients with moderate to severe renal dysfunction.

Mechanical methods (ICD and GCS) are recommended when anticoagulation is contraindicated or as an adjunct to anticoagulation in the very high-risk patients [254-256]. In very high-risk patients, LMWH is preferred over UFH [257-259]. Patients receiving heparin should be monitored for development of heparin-induced thrombocytopenia (HIT).

\section{G. Stress Ulcer Prophylaxis (SUP)}

We recommend that stress ulcer prophylaxis using $\mathrm{H}_{2}$ blocker (Grade 1A) or proton pump inhibitor PPI (Grade 1B) be given to patients with severe sepsis to prevent upper GI bleed. Benefit of prevention of upper GI bleed must be weighed against potential effect of an increased stomach $\mathrm{pH}$ on development of ventilator-associated pneumonia.

Rationale. Although no study has been performed specifically in patients with severe sepsis, trials confirming the benefit of stress ulcer prophylaxis reducing upper GI bleeds in general ICU populations would suggest that $20-25 \%$ of patients enrolled in these types of trials have sepsis [260-263]. This benefit should be applicable to patients with severe sepsis and septic shock. In addition, the conditions shown to benefit from stress ulcer prophylaxis (coagulopathy, mechanical ventilation, hypotension) are frequently present in patients with severe sepsis and septic shock [264, 265].

Although there are individual trials that have not shown benefit from SUP, numerous trials and a metaanalysis show reduction in clinically significant upper GI bleeding, which we consider significant even in the absence of proven mortality benefit [266-269]. The benefit of prevention of upper GI bleed must be weighed against the potential effect of increased stomach $\mathrm{pH}$ on greater incidence of ventilator-associated pneumonia [270]. Those severe sepsis patients with the greatest risk of upper GI bleeding are likely to benefit most from stress ulcer prophylaxis. The rationale for the preference for suppression of acid production over sulcrafate was based on the study of 1200 patients by Cook et al comparing $\mathrm{H}_{2}$ blockers and sucralfate and a meta-analysis [271, 272]. 2 studies support equivalency between $\mathrm{H}_{2}$ blockers and PPIs. One was in very ill ICU patients. The second study is larger and demonstrates non-inferiority of omeprazole suspension for clinically significant stress ulcer bleeding [273, 274]. No data relating to utility of enteral feeding in stress ulcer prophylaxis exist. Patients should be periodically evaluated for continued need for prophylaxis.

\section{H. Selective Digestive Tract Decontamination (SDD)}

The guidelines group was evenly split on the issue of SDD, with equal numbers weakly in favor and against recommending the use of SDD (see appendix H). The committee therefore chose not to make a recommendation for the use of SDD specifically in severe sepsis at this time. The final consensus on use of SDD in severe sepsis was achieved at the last nominal committee meeting and subsequently approved by the entire committee (see Appendix $\mathrm{H}$ for committee vote).

Rationale. The cumulative conclusion from the literature demonstrates that prophylactic use of SDD (enteral non-absorbable antimicrobials and short-course intravenous antibiotics) reduces infections, mainly pneumonia, and mortality in the general population of critically ill and trauma patients [275-286] without promoting emergence of resistant Gram negative bacteria. Post hoc subgroup analyses [287, 288] of two prospective blinded studies [289, 290] suggest that SDD reduces nosocomial (secondary) infections in ICU patients admitted with primary infections [268] and may reduce mortality [288]. No studies of SDD specifically focused on patients with severe sepsis or septic shock. The use of SDD in severe sepsis patients would be targeted toward preventing secondary infection. As the main effect of SDD is in preventing ventilator-associated pneumonia (VAP), studies comparing SDD with non-antimicrobial interventions such as ventilator bundles for reducing VAP are needed. Further investigation is required to determine the comparative efficacy of these two interventions, separately or in combination. Although studies incorporating enteral vancomycin in the regimen appear to be safe [291, 292, 293] concerns persist about the potential for emergence of resistant Gram positive infections.

\section{Consideration for Limitation of Support}

We recommend that advance care planning, including the communication of likely outcomes and realistic goals of treatment, be discussed with patients and families (Grade 1D).

Rationale. Decisions for less aggressive support or withdrawal of support may be in the patient's best interest. [294-296] Too frequently, inadequate physician/family communication characterizes end-of-life care in the ICU. The level of life support given to ICU patients may not be consistent with their wishes. Early and frequent caregiver discussions with patients who face death in the ICU and with their loved ones may facilitate appropriate application and withdrawal of life-sustaining therapies. A recent RCT demonstrated reduction of anxiety and depression in family members when end-of-life meetings were carefully planned, conducted, included advance care planning, and provided relevant information about diagnosis, prognosis, and treatment [297]. 


\section{Pediatric Considerations in Severe Sepsis}

While sepsis in children is a major cause of mortality, the overall mortality from severe sepsis in children is much lower that that in adults, estimated at about 10\% [298]. The definitions for severe sepsis and septic shock in children are similar but not identical to the definitions in adults [299]. In addition to age-appropriate differences in vital signs, the definition of systemic inflammatory response syndrome requires the presence of either temperature or leukocyte abnormalities. The presence of severe sepsis requires sepsis plus cardiovascular dysfunction or ARDS or 2 or more other organ dysfunctions [299].

\section{A. Antibiotics}

1. We recommend antibiotics be administered within one hour of the identification of severe sepsis, after appropriate cultures have been obtained (Grade 1D).

Early antibiotic therapy is as critical for children with severe sepsis as it is for adults.

\section{B. Mechanical Ventilation}

No graded recommendations.

Due to low functional residual capacity, young infants and neonates with severe sepsis may require early intubation [300]. Drugs used for intubation have important side effects in these patients, for example, concerns have been raised about the safety of using etomidate in children with meningococcal sepsis because of adrenal suppression effect [301]. The principles of lung-protective strategies are applied to children as they are to adults.

\section{Fluid Resuscitation}

1. We suggest initial resuscitation begin with infusion of crystalloids with boluses of $20 \mathrm{~mL} / \mathrm{kg}$ over 5-10 minutes, titrated to clinical monitors of cardiac output, including heart rate, urine output, capillary refill, and level of consciousness (Grade 2C).

Intravenous access for fluid resuscitation and inotrope/vasopressor infusion is more difficult to attain in children than in adults. The American Heart Association along with the American Academy of Pediatrics has developed pediatric advanced life support guidelines for emergency establishment of intravascular support encouraging early intraosseous access [302]. On the basis of a number of studies, it is accepted that aggressive fluid resuscitation with crystalloids or colloids is of fundamental importance to survival of septic shock in children [303-308]. Three randomized, controlled trials compare the use of colloid to crystalloid resuscitation in children with dengue shock [303, 307, 308]. No difference in mortality between colloid or crystalloid resuscitation was shown.

Children normally have a lower blood pressure than adults, and fall in blood pressure can be prevented by vasoconstriction and increasing heart rate. Therefore, blood pressure by itself is not a reliable end point for assessing the adequacy of resuscitation. However, once hypotension occurs, cardiovascular collapse may soon follow. Hepatomegaly occurs in children who are fluid overloaded and can be a helpful sign of adequacy of fluid resuscitation. Large fluid deficits typically exist and initial volume resuscitation usually requires $40-60 \mathrm{~mL} / \mathrm{kg}$ but can be much higher [304-308]. However, the rate of fluid administration should be reduced substantially when there are (clinical) signs of adequate cardiac filling without hemodynamic improvement.

\section{Vasopressors/Inotropes (should be used in volume loaded patients with fluid refractory shock)}

1. We suggest dopamine as the first choice of support for the pediatric patient with hypotension refractory to fluid resuscitation (Grade 2C).

In the initial resuscitation phase, vasopressor therapy may be required to sustain perfusion pressure, even when hypovolemia has not yet been resolved. Children with severe sepsis can present with low cardiac output and high systemic vascular resistance, high cardiac output and low systemic vascular resistance, or low cardiac output and low systemic vascular resistance shock. At various stages of sepsis or the treatment thereof, a child may move from one hemodynamic state to another. Vasopressor or inotrope therapy should be used according to the clinical state of the child.

Dopamine-refractory shock may reverse with epinephrine or norepinephrine infusion [309].

2. We suggest that patients with low cardiac output and elevated systemic vascular resistance states (cool extremities, prolonged capillary refill, decreased urine output but normal blood pressure following fluid resuscitation) be given dobutamine (Grade 2C).

The choice of vasoactive agent is determined by the clinical examination. For the child with a persistent low cardiac output state with high systemic vascular resistance despite fluid resuscitation and inotropic support, vasodilator therapy may reverse shock [310]. When pediatric patients remain in a normotensive low cardiac output and 
high vascular resistance state despite epinephrine and vasodilator therapy, the use of a phosphodiesterase inhibitor may be considered [311-313]. In the case of extremely low systemic vascular resistance despite the use of norepinephrine, vasopressin use has been described in a number of case-reports. Thus far there is no clear evidence for the use of vasopressin in pediatric sepsis [314, 315].

\section{E. Therapeutic End Points}

1. We suggest that the therapeutic end points of resuscitation of septic shock be normalization of the heart rate, capillary refill of $<2$ secs, normal pulses with no differential between peripheral and central pulses, warm extremities, urine output $>1 \mathrm{~mL} \cdot \mathrm{kg}^{-1} \cdot \mathrm{hr}^{-1}$, and normal mental status [290] (Grade 2C).

Capillary refill may be less reliable in a cold environment. Other end points that have been widely used in adults and may logically apply to children include decreased lactate and improved base deficit, $\mathrm{ScvO}_{2} \geq 70 \%$ or $\mathrm{SvO}_{2} \geq 65 \%$, CVP of 8-12 mm Hg or other methods to analyze cardiac filling. Optimizing preload optimizes cardiac index. When using measurements to assist in identifying acceptable cardiac output in children with systemic arterial hypoxemia such as cyanotic congenital heart disease or severe pulmonary disease, arterial-venous oxygen content difference is a better marker than mixed venous hemoglobin saturation with oxygen. As noted previously, blood pressure by itself is not a reliable end point for resuscitation. If a thermodilution catheter is used, therapeutic end points are cardiac index $>3.3$ and $<6.0 \mathrm{~L} \cdot \mathrm{min}^{-1} \cdot \mathrm{m}^{-2}$ with normal coronary perfusion pressure (mean arterial pressure - central venous pressure) for age. [290] Using clinical endpoints such as reversal of hypotension and restoration of capillary refill for initial resuscitation at the community hospital level before transfer to a tertiary center was associated with significantly improved survival rates in children with septic shock [305]. Development of a transport system including publicizing to local hospitals and transport with mobile intensive care services significantly decreased the case fatality rate from meningococcal disease in the United Kingdom [316].

\section{F. Approach to Pediatric Septic Shock}

Figure 1 shows a flow diagram summarizing an approach to pediatric septic shock [317].

\section{G. Steroids}

1. We suggest that hydrocortisone therapy be reserved for use in children with catecholamine resistance and suspected or proven adrenal insufficiency (Grade 2C).
Patients at risk for adrenal insufficiency include children with severe septic shock and purpura [318, 319], children who have previously received steroid therapies for chronic illness, and children with pituitary or adrenal abnormalities. Children who have clear risk factors for adrenal insufficiency should be treated with stress dose steroids (hydrocortisone $50 \mathrm{mg} / \mathrm{m}^{2} / 24 \mathrm{hr}$ ).

Adrenal insufficiency in pediatric severe sepsis is associated with a poor prognosis [320]. No strict definitions exist, but absolute adrenal insufficiency in the case of catecholamine-resistant septic shock is assumed at a random total cortisol concentration $<18 \mu \mathrm{g} / \mathrm{dL}$ $(496 \mathrm{nmol} / \mathrm{L})$. A post $30-$ or $60-\mathrm{min}$ ACTH stimulation test increase in cortisol of $\leq 9 \mu \mathrm{g} / \mathrm{dL}(248 \mathrm{mmol} / \mathrm{L})$ has been used to define relative adrenal insufficiency. The treatment of relative adrenal insufficiency in children with septic shock is controversial. A retrospective study from a large administrative database recently reported that the use of any corticosteroids in children with severe sepsis was associated with increased mortality (OR 1.9 95\% CI 1.7-2.2) [321]. While steroids may have been given preferentially to more severely ill children, the use of steroids was an independent predictor of mortality in multivariable analysis [321]. Given the lack of data in children and potential risk, steroids should not be used in those children who do not meet minimal criteria for adrenal insufficiency. A randomized, controlled trial in children with septic shock is very much needed.

\section{H. Protein C and Activated Protein C}

1. We recommend against the use rhAPC in children (Grade 1B).

Protein $\mathrm{C}$ concentrations in children reach adult values at the age of 3 yrs. This might indicate that the importance of protein $\mathrm{C}$ supplementation either as protein C concentrate or as rhAPC is even greater in young children than in adults [322]. There has been one dose finding, randomized, placebo-controlled study performed using protein $\mathrm{C}$ concentrate. This study was not powered to show an effect on mortality rate, but did show a positive effect on sepsis-induced coagulation disturbances [323]. An RCT of rhAPC in pediatric severe sepsis patients was stopped by recommendation of the Data Monitoring Committee for futility after enrollment of 399 patients. 28-day all cause mortality: 18\% placebo group vs. 17\% APC group. Major amputations occurred in $3 \%$ of the placebo group vs. $2 \%$ in the APC group [324]. Due to the increased risk of bleeding (7\% vs. $6 \%$ in the pediatric trial) and lack of proof of efficacy, rhAPC is not recommended for use in children. 
Fig. 1 Approach to Pediatric Shock

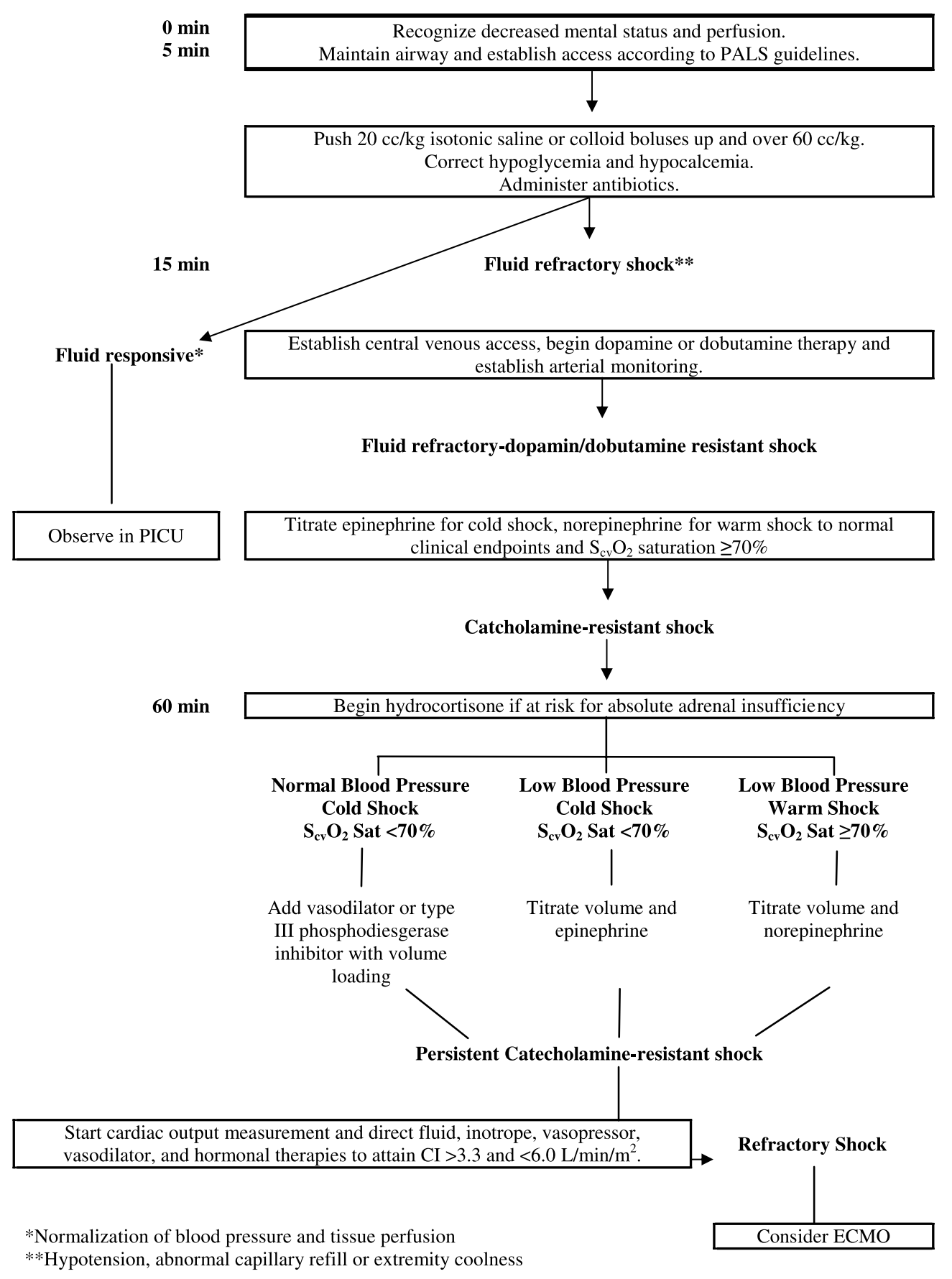

\section{DVT Prophylaxis}

1. We suggest the use of DVT prophylaxis in postpubertal children with severe sepsis (Grade 2C).

Most DVTs in young children are associated with central venous catheters. Femoral venous catheters are commonly used in children, and central venous catheterassociated DVTs occur in approximately $25 \%$ of children with a femoral central venous catheter. Heparin-bonded catheters may decrease the risk of catheter-associated DVT and should be considered for use in children with severe sepsis. [325, 326] No data on the efficacy of unfractionated or low-molecular weight heparin prophylaxis to prevent catheter-related DVT in children in the ICU exist. 


\section{J. Stress Ulcer Prophylaxis}

No graded recommendations.

Studies have shown that the rate of clinically important gastrointestinal bleeding in children occurs at rates similar to adults [327, 328]. As in adults, coagulopathy and mechanical ventilation are risk factors for clinically important gastrointestinal bleeding. Stress ulcer prophylaxis strategy is commonly used in mechanically-ventilated children, usually with $\mathrm{H}_{2}$ blockers. Its effect is not known.

\section{K. Renal Replacement Therapy}

No graded recommendations.

Continuous veno-venous hemofiltration (CVVH) may be clinically useful in children with anuria/severe oliguria and fluid overload, but no large RCTs have been performed comparing CVVH with intermittent dialysis. A retrospective study of 113 critically ill children reported that children with less fluid overload before CVVH had better survival, especially in those children with dysfunction of 3 or more organs [329]. CVVH or other renal replacement therapy should be instituted in children with anuria/severe oliguria before significant fluid overload occurs.

\section{Glycemic Control}

No graded recommendations.

In general, infants are at risk for developing hypoglycemia when they depend on intravenous fluids. This means that a glucose intake of $4-6 \mathrm{mg} \cdot \mathrm{kg}^{-1} \cdot \mathrm{min}^{-1}$ or maintenance fluid intake with glucose $10 \% / \mathrm{NaCl}$ containing solution is advised. Associations have been reported between hyperglycemia and an increased risk of death and longer length of stay [330]. A recent retrospective PICU study reported associations of hyperglycemia, hypoglycemia, and glucose variability with length of stay and mortality rates. [331] No studies in pediatric patients (without diabetes mellitus) analyzing the effect of strict glycemic control using insulin exist. In adults, the recommendation is to maintain a serum glucose below $150 \mathrm{mg} / \mathrm{dL}$. Insulin therapy to avoid long periods of hyperglycemia seems sensible in children as well, but the optimal goal glucose is not known. However, continuous insulin therapy should only be done with frequent glucose monitoring in view of the risks for hypoglycemia.

\section{Sedation/Analgesia}

1. We recommend sedation protocols with a sedation goal when sedation of critically ill mechanically ventilated patients with sepsis is required (Grade 1D).
Appropriate sedation and analgesia are the standard of care for children who are mechanically ventilated. Although there are no data supporting any particular drugs or regimens, it should be noted that propofol should not be used for long term sedation in children because of the reported association with fatal metabolic acidosis [332, 333].

\section{N. Blood Products}

No graded recommendations.

The optimal hemoglobin for a critically ill child with severe sepsis is not known. A recent multicenter trial reported similar outcomes in stable critically ill children managed with a transfusion threshold of $7 \mathrm{gm} / \mathrm{dl} \mathrm{com-}$ pared to those managed with a transfusion threshold of $9.5 \mathrm{~g} / \mathrm{dL}$ [334]. Whether a lower transfusion trigger is safe or appropriate in the initial resuscitation of septic shock has not been determined.

\section{O. Intravenous Immunoglobulin}

1. We suggest that immunoglobulin may be considered in children with severe sepsis (Grade 2C).

Administration of polyclonal intravenous immunoglobulin has been reported to reduce mortality rate and is a promising adjuvant in the treatment of sepsis and septic shock in neonates. A recent randomized controlled study of polyclonal immunoglobulin in pediatric sepsis syndrome patients $(n=100)$, showed a significant reduction in mortality, LOS, and less progress to complications, especially DIC [335].

\section{P. Extracorporeal membrane oxygenation (ECMO)}

1. We suggest that use of ECMO be limited to refractory pediatric septic shock and/or respiratory failure that cannot be supported by conventional therapies (Grade 2C).

ECMO has been used in septic shock in children, but its impact is not clear. Survival from refractory shock or respiratory failure associated with sepsis is $80 \%$ in neonates and $50 \%$ in children. In one study analyzing 12 patients with meningococcal sepsis in ECMO, eight of the 12 patients survived, with six leading functionally normal lives at a median of $1 \mathrm{yr}$ (range, 4 months to $4 \mathrm{yrs}$ ) of follow-up. Children with sepsis on ECMO do not perform worse than children without sepsis at long-term follow-up [336, 337].

Although the pediatric considerations section of this manuscript offers important information to the practicing pediatric clinician for the management of critically ill children with sepsis, the reader is referred to the references at the end of the document for more in-depth descriptions of appropriate management of pediatric septic patients. 


\section{Summary and Future Directions}

The reader is reminded that although this document is static, the optimum treatment of severe sepsis and septic shock is a dynamic and evolving process. New interventions will be proven and established interventions, as stated in the current recommendations, may need modification. This publication represents an ongoing process. The Surviving Sepsis Campaign and the consensus committee members are committed to updating the guidelines on a regular basis as new interventions are tested and published in the literature.

Although evidence-based recommendations have been frequently published in the medical literature, documentation of impact on patient outcome is limited [338]. There is, however, growing evidence that protocol implementation associated with education and performance feedback does change clinician behavior and may improve outcomes in and reduce costs in severe sepsis [20, 24, 25]. Phase III of the Surviving Sepsis Campaign targets the implementation of a core set of the previous recommendations in hospital environments where change in behavior and clinical impact are being measured. The sepsis bundles were developed in collaboration with the Institute of Healthcare Improvement [339]. Concurrent or retrospective chart review will identify and track changes in practice and clinical outcome. Software and software support is available at no cost in 7 languages, allowing bedside data entry and allows creation of regular reports for performance feedback. The Campaign also offers significant program support and educational materials at no cost to the user (www.survivingsepsis.org ).

Engendering evidence-based change in clinical practice through multi-faceted strategies while auditing practice and providing feedback to healthcare practitioners is the key to improving outcomes in severe sepsis. Nowhere is this more evident than in the worldwide enthusiasm for Phase III of the Campaign, a performance improvement program using SSC guideline-based sepsis bundles. Using the guidelines as the basis, the bundles have established a global best practice for the management of critically ill patients with severe sepsis. As of November 2007, over 12,000 patients have been entered into the SSC central database, representing the efforts of 239 hospitals in 17 countries. Change in practice and potential effect on survival are being measured.
Acknowledgment of Support As mentioned above in the methods section, the Surviving Sepsis Campaign (SSC) is partially funded by unrestricted educational industry grants, including those from Edwards LifeSciences, Eli Lilly and Company, and Philips Medical Systems. SSC also received funding from the Coalition for Critical Care Excellence of the Society of Critical Care Medicine. The great majority of industry funding has come from Eli Lilly and Company.

Current industry funding for the Surviving Sepsis Campaign is directed to the performance improvement initiative. No industry funding was used for committee meetings. No honoraria were provided to committee members. The revision process was funded primarily by the Society of Critical Care Medicine, with the sponsoring professional organizations providing travel expenses for their designated delegate to the guidelines revision meeting where needed.

Other Acknowledgements Toni Piper and Rae McMorrow for their assistance in bringing the manuscript together. Gordon Guyatt and Henry Masur, M.D. for their guidance on grading of evidence and antibiotic recommendations respectively.

Nine of the 11 organizations that sponsored the first guidelines are sponsors of the revision. Four additional national organizations (Canadian Critical Care Society, Japanese Association for Acute Medicine, Japanese Society of Intensive Care Medicine, and Society of Hospital Medicine), the World Federation of Societies of Intensive and Critical Care Medicine and two sepsis organizations (German Sepsis Society and the Latin American Sepsis Institute) have also come on board as sponsors. Two organizations that sponsored the first guidelines (American Thoracic Society and Australian and New Zealand Intensive Care Society) elected not to sponsor the revision.

\section{A. Source Control}

\section{Source Control Examples}

Technique

\begin{tabular}{ll}
\hline Drainage & - Intra-abdominal abscess \\
& - Thoracic empyema \\
Debridement & - Septic arthritis \\
& - Infected pancreatic necrosis \\
& - Intestinal infarction \\
Device & - Infectiastinitis \\
removal & - Urinary catheter \\
Definitive & - Infected intrauterine contraceptive device \\
control & - Cholecystectomy for gangrenous cholecystitis \\
& - Amputation for clostridial myonecrosis
\end{tabular}




\section{B. Steroids}

Considerable difference of opinion existed among committee members as to the best option for the style of the steroid in septic shock recommendations. Some committee members argued for two recommendations and pointed to the two distinct patient populations of (1) the French Trial (enrollment early in septic shock and blood pressure unresponsive to vasopressors) and (2) the CORTICUS trial (enrollment allowed up to $72 \mathrm{hrs}$ and did not target patients with blood pressure unresponsive to vasopressin), leading to two distinct results. Furthermore, a single recommendation suggested to some that this approach might lead to excessive use of steroids and increased incidence of super-infections, citing the sepsis and septic shock adverse events in the steroid treated patients in the CORTICUS trial. Those that argued for one recommendation pointed to problems with two different recommendations that would require the bedside clinician to choose a time point for classification of one or the other as well as a distinct blood pressure cut-off with the potential for the blood pressure to vary over time. In addition there is inadequate data to provide standardization of how much fluids and vasopressors should be in place to call the blood pressure unresponsive or poorly responsive. They also pointed to the fact that the increased super-infection/sepsis/septic shock adverse events in CORTICUS are contrary to the results of other stress dose steroid trials such as early ARDS (lower incidence of infections) (341), late ARDS (decreased development of septic shock) (342), and community-acquired pneumonia (decreased development of septic shock) (114). Based on GRADE adjudication guidelines, a secret ballot vote was conducted to resolve the issue.

The two options put to vote were:

\section{Two recommendation option}

1. We suggest intravenous hydrocortisone be given to adult septic shock patients if blood pressure is inadequate with appropriate fluid resuscitation and vasopressor therapy (Grade 2B).

2. We suggest intravenous hydrocortisone not be given to adult septic shock patients if blood pressure is adequate with appropriate fluid resuscitation and vasopressor therapy (Grade 2B).

\section{One recommendation option}

1. We suggest intravenous hydrocortisone be given only to adult septic shock patients with blood pressure poorly responsive to fluid resuscitation and vasopressor therapy (Grade 2C).

The committee vote that determined the current recommendation was:

Favor two recommendation option - 19

Favor one recommendation option - 31

Abstain - 1

\section{Contraindications to use of recombinant human activated protein C (rhAPC)}

rhAPC increases the risk of bleeding. rhAPC is contraindicated in patients with the following clinical situations in which bleeding could be associated with a high risk of death or significant morbidity.

- Active internal bleeding

- Recent (within 3 months) hemorrhagic stroke

- Recent (within 2 months) intracranial or intraspinal surgery, or severe head trauma

- Trauma with an increased risk of life-threatening bleeding

- Presence of an epidural catheter

- Intracranial neoplasm or mass lesion or evidence of cerebral herniation

- Known hypersensitivity to rhAPC or any component of the product

See labeling instructions for relative contraindications. The committee recommends that platelet count be maintained at $\geq 30,000$ or greater during infusion of rhAPC.

Physicians' Desk Reference. 61st Edition. Montvale, NJ, Thompson PDR, 2007, p 1829

\section{Recombinant Activated Protein C Nominal Group Vote}

\begin{tabular}{lllll}
\hline $\begin{array}{l}\text { Strong } \\
\text { for use }\end{array}$ & $\begin{array}{l}\text { Weak } \\
\text { for use }\end{array}$ & Neutral & $\begin{array}{l}\text { Weak for } \\
\text { not using }\end{array}$ & $\begin{array}{l}\text { Strong for } \\
\text { not using }\end{array}$ \\
\hline 6 & 15 & 1 & 0 & 0 \\
\hline
\end{tabular}




\section{E. ARDSNET Ventilator Management (96)}

- Assist control mode - volume ventilation

- Reduce tidal volume to $6 \mathrm{~mL} / \mathrm{kg}$ lean body weight

- Keep inspiratory plateau pressure (Pplat) $\leq 30 \mathrm{~cm} \mathrm{H}_{2} \mathrm{O}$

- Reduce TV as low as $4 \mathrm{~mL} / \mathrm{kg}$ predicted body weight to limit Pplat

- Maintain $\mathrm{SaO}_{2} / \mathrm{SpO}_{2} 88-95 \%$

- Anticipated PEEP settings at various $\mathrm{FIO}_{2}$ requirements

$\begin{array}{lllllllll}\mathrm{FiO}_{2} & 0.3 & 0.4 & 0.4 & 0.5 & 0.5 & 0.6 & 0.7 & 0.7\end{array}$

$\begin{array}{lllll}\text { PEEP } 5 & 5 & 8 & 8 & 10\end{array}$

* Predicted Body Weight Calculation

- Male $-50+2.3$ (height (inches) -60 ) or $50+0.91$ (height $(\mathrm{cm})-152.4$ )

- Female $-45.5+2.3$ (height (inches) -60$)$ or $45.5+0.91$ (height $(\mathrm{cm})-152.4$ )

$T V$, tidal volume; $\mathrm{SaO}_{2}$, arterial oxygen saturation; $\mathrm{SpO}_{2}$, pulse oximetry oxyhemoglobin saturation;

$P E E P$, positive end-expiratory pressure

\section{F. Use of spontaneous breathing trial in weaning ARDS patients}

Original illness resolving; no new illness

Off vasopressors and continuous sedatives

Cough during suctioning

$\mathrm{PaO}_{2} / \mathrm{F}_{\mathrm{I}} \mathrm{O}_{2}>200$

PEEP $\leq 5 \mathrm{~cm} \mathrm{H}_{2} \mathrm{O}$

Minute ventilation $<15 \mathrm{~L}$ min

Frequency/tidal volume (F/TV) ratio $\leq 105$ during

two-minute spontaneous breathing trial

$$
\downarrow
$$

Spontaneous Breathing Trial * (30 to 120 minutes)

Respiratory rate $>35$

Oxygen saturation $<90$

Pulse $>140 / \mathrm{min}$ or change $\geq 20 \%$

SBP $>180 \mathrm{~mm} \mathrm{Hg}$ or $<90 \mathrm{~mm} \mathrm{Hg}$

Agitation, diaphoresis, or anxiety F/TV ratio $>105$

Note: Achieving any of these criteria for a sustained period at any time during the trial represents a weaning

failure and the need to return to maintenance MV.

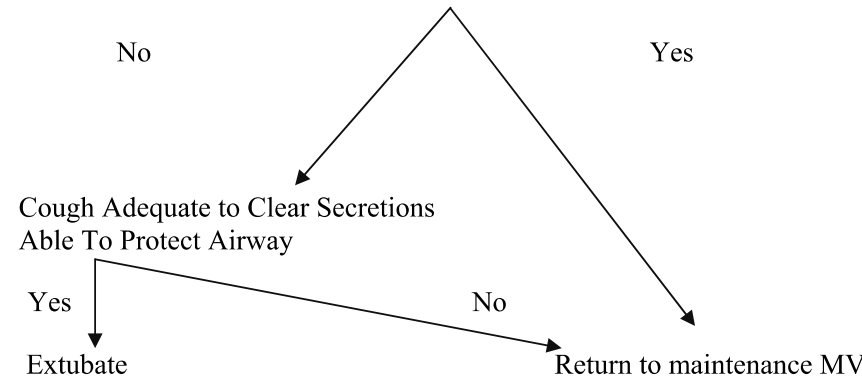

$P E E P$, positive end-expiratory pressure; $F / T V$, frequency/tidal volume; $S B P$, systolic blood pressure; $M V$, mechanical ventilation; ${ }^{*}$ Options include T-Piece, continuous positive airway pressure $5 \mathrm{~cm} \mathrm{H}_{2} \mathrm{O}$ or low level (5-10 $\mathrm{cm} \mathrm{H}_{2} \mathrm{O}$ typically based on ET tube size) pressure support ventilation (167-170)

\section{G. Glycemic control committee vote}

Glycemic Control - 90\%

Total votes $=51$

Agree - 34

Too conservative, but accept -4

Too liberal, but accept -8

Disapprove, too conservative - 0

Disapprove, too liberal - 5

Disapprove, other - 0

\section{H. Selective Digestive Decontamination Nominal Group Vote}

\begin{tabular}{llllll}
\hline Antibiotics & $\begin{array}{l}\text { Strong } \\
\text { for use }\end{array}$ & $\begin{array}{l}\text { Weak } \\
\text { for use }\end{array}$ & Neutral & $\begin{array}{l}\text { Weak for } \\
\text { not using }\end{array}$ & $\begin{array}{l}\text { Strong for } \\
\text { not using }\end{array}$ \\
\hline Syst and oral & - & 9 & 4 & 8 & 1 \\
Syst alone & - & 2 & 7 & 5 & 3 \\
\hline
\end{tabular}

Syst, systemic

\section{2008 SSC Guidelines Committee}

R. Phillip Dellinger (Chair), Tom Ahrens ${ }^{\mathrm{a}}$, Naoki Aikawa $^{\mathrm{b}}$, Derek Angus, Djillali Annane, Richard Beale, Gordon R. Bernard, Julian Bion ${ }^{\mathrm{c}}$, Christian BrunBuisson, Thierry Calandra, Joseph Carcillo, Jean Carlet ${ }^{\mathrm{c}}$, Terry Clemmer, Jonathan Cohen, Edwin A. Deitch ${ }^{\mathrm{d}}$, Jean-Francois Dhainaut, Mitchell Fink, Satoshi Gando ${ }^{b}$, Herwig Gerlach ${ }^{\mathrm{c}}$, Gordon Guyatt ${ }^{\mathrm{e}}$, Maurene Harvey, Jan Hazelzet, Hiroyuki Hirasawa ${ }^{f}$, Steven M. Hollenberg, Michael Howell, Roman Jaeschke ${ }^{\mathrm{e}}$, Robert Kacmarek, Didier Keh, Mitchell M. Levy ${ }^{\mathrm{g}}$, Jeffrey Lipman, John J. Marini, John Marshall, Claude Martin ${ }^{\mathrm{c}}$, Henry Masur, Steven Opal, Tiffany M Osborn ${ }^{\text {h }}$, Giuseppe Pagliarello ${ }^{\text {, }}$, Margaret Parker, Joseph Parrillo, Graham Ramsay ${ }^{\mathrm{c}}$, 
Adrienne Randolph, Marco Ranieri ${ }^{\mathrm{c}}$, Robert C. Read ${ }^{\mathrm{j}}$, Konrad Reinhart ${ }^{\mathrm{k}}$, Andrew Rhodes ${ }^{\mathrm{c}}$, Emmanuel Rivers ${ }^{\mathrm{h}}$, Gordon Rubenfeld, Jonathan Sevransky, Eliezer Silva ', Charles L. Sprung c, B. Taylor Thompson, Sean R. Townsend, Jeffery Vender ${ }^{\mathrm{m}}$, Jean-Louis Vincent ${ }^{\mathrm{n}}$, Tobias Welte $^{\circ}$, Janice Zimmerman

a - American Association of Critical-Care Nurses

$\mathrm{b}$ - Japanese Association for Acute Medicine

c - European Society of Intensive Care Medicine

d - Surgical Infection Society

e - Grades of Recommendation, Assessment, Development and Evaluation (GRADE) Group

f - Japanese Society of Intensive Care Medicine

$\mathrm{g}$ - Society of Critical Care Medicine

$\mathrm{h}$ - American College of Emergency Physicians

i - Canadian Critical Care Society

j - European Society of Clinical Microbiology and Infectious Diseases

k - German Sepsis Society

1 - Latin American Sepsis Institute

$\mathrm{m}$ - American College of Chest Physicians

$\mathrm{n}$ - International Sepsis Forum

o - European Respiratory Society

\section{J. Author Disclosure Information 2006-2007}

Dr. Dellinger has consulted for AstraZeneca, Talecris, and B Braun. He has received honoraria from Eli Lilly (2), Brahms (2), INO Therapeutics (1), Pulsion (1), and bioMerieux (1). He has also received grant support from AstraZeneca and Artisan.

Dr. Levy has received honoraria from Eli Lilly and Edwards Lifesciences. He has also received grant support from Phillips Medical Systems, Edwards Lifesciences, Phillips Medical Systems, Novartis, Biosite, and Eisai.

Dr. Carlet has consulted for Forrest, Wyeth, Chiron, bioMerieux, and GlaxoSmithKline. He has also received honoraria from Eli Lilly, Becton Dickinson, Jansen, Cook, AstraZeneca, Hutchinson, Bayer, Gilead, MSD, and Targanta.

Dr. Bion has not disclosed any potential conflicts of interest.

Dr. Parker has consulted for Johnson \& Johnson.

Dr. Jaeschke has received honoraria from AstraZeneca, Boehringer, Eli Lilly, GlaxoSmithKline, and MSD.

Dr. Reinhart has consulted for Eli Lilly and Edwards Lifesciences. He has also received honoraria from B Braun and royalties from Edwards Lifesciences.
Dr. Angus has consulted for or received speaking fees from AstraZeneca, Brahms Diagnostica, Eisai, Eli Lilly, GlaxoSmithKline, OrthoBiotech, Takeda, and Wyeth-Ayerst. He has also received grant support from GlaxoSmithKline, OrthoBiotech, and Amgen.

Dr. Brun-Buisson has not disclosed any potential conflicts of interest.

Dr. Beale has received honoraria from Eisai and speaking fees (paid to university) from Lilly UK, Philips, Lidco, and Chiron.

Dr. Calandra has consulted for Baxter, received honoraria from Roche Diagnostics, and grant support from Baxter and Roche Diagnostics. He also served on the advisory board for Biosite.

Dr. Dhainaut has consulted for Eli Lilly and Novartis. He has also received honoraria from Eli Lilly.

Dr. Gerlach has not disclosed any potential conflicts of interest.

Ms. Harvey has not disclosed any potential conflicts of interest.

Dr. Marini has consulted for KCI and received honoraria from Maquet.

Dr. Marshall has consulted for Becton-Dickinson, Takeda, Pfizer, Spectral Diagnostics, Eisai, and LeoPharma. He has also received honoraria from Spectral Diagnostics.

Dr. Ranieri has served on the advisory board for Maquet and received support for a sponsored trial from Eli Lilly. He has also received grant support from Tyco, Draeger, and Hamilton.

Dr. Ramsay has consulted for Edwards Lifesciences and Respironics.

Dr. Sevransky has not disclosed any potential conflicts of interest.

Dr. Thompson has consulted for Eli Lilly, Abbott, and AstraZeneca. He has also received grant support from the NIH for a study on computerized glucose control.

Dr. Townsend has not disclosed any potential conflicts of interest.

Dr. Vender has consulted and received honoraria from Eli Lilly.

Dr. Zimmerman has not disclosed any potential conflicts of interest.

Dr. Vincent has consulted for AstraZeneca, Biosite, bioMerieux, Edwards Lifesciences, Eli Lilly Eisai, Ferring, GlaxoSmithKline, Intercell, Merck, Novartis, NovoNordisk, Organon, Pfizer, Phillips Medical Systems, Roche Diagnostics, Spectral Diagnostics, Takeda, and WyethLederle. He has also received honoraria from Eli Lilly, Edwards Lifesciences, Eisai, GlaxoSmithKline, Novartis, NovoNordisk, and Pfizer. 


\section{References}

\section{Introduction and Methods}

1. Angus DC, Linde-Zwirble WT, Lidicker J, Clermont G, Carcillo J, Pinsky MR (2001) Epidemiology of severe sepsis in the United States: analysis of incidence, outcome, and associated costs of care. Crit Care Med 29:1303-1310

2. Dellinger RP (2003) Cardiovascular management of septic shock. Crit Care Med 31:946-955

3. Martin GS, Mannino DM, Eaton S, Moss M (2003) The Epidemiology of Sepsis in the United States from 1979 through 2000. N Engl J Med 348:1546-1554

4. Linde-Zwirble WT, Angus DC (2004) Severe sepsis epidemiology: sampling, selection, and society. Crit Care 8:222-226

5. Dombrovskiy VY, Martin AA, Sunderram J, Paz HL (2007) Rapid increase in hospitalization and mortality rates for severe sepsis in the United States: a trend analysis from 1993 to 2003. Crit Care Med 35:1414-1415

6. Dellinger RP, Carlet JM, Masur H, Gerlach H, Calandra T, Cohen J, GeaBanacloche J, Keh D, Marshall JC, Parker MM, Ramsay G, Zimmerman JL, Vincent JL, Levy MM, Surviving Sepsis Campaign Management Guidelines Committee (2004) Surviving Sepsis Campaign guidelines for management of severe sepsis and septic shock. Crit Care Med 32:858-873

7. Dellinger RP, Carlet JM, Masur H, Gerlach H, Calandra T, Cohen J, GeaBanacloche J, Keh D, Marshall JC, Parker MM, Ramsay G, Zimmerman JL, Vincent JL, Levy MM, Surviving Sepsis Campaign Management Guidelines Committee (2004) Surviving Sepsis Campaign guidelines for management of severe sepsis and septic shock. Intensive Care Med 30:536-555

8. Guyatt G, Schünemann H, Cook D, Jaeschke R, Pauker S (2004) Applying the grades of recommendations for antithrombotic and thrombolytic therapy: The seventh ACCP conference of antithrombotic and thrombolytic therapy. Chest 126:179S-187S

9. GRADE working group (2004) Grading quality of evidence and strength of recommendations. BMJ 328:1490-1498
10. Guyatt G, Gutterman D, Baumann MH, Addrizzo-Harris D, Hylek EM, Phillips B (2006) Grading strength of recommendations and quality of evidence in clinical guidelines: report from an American College of Chest Physicians task force. Chest 129:174-181

11. Schünemann HJ, Jaeschke R, Cook DJ, Bria WF, El-Solh AA, Ernst A, Fahy BF, Gould MK, Horan KL, Krishnan JA, Manthous CA, Maurer JR, McNicholas WT, Oxman AD, Rubenfeld G, Turino GM, Guyatt G, ATS Documents Development and Implementation Committee (2006) An Official ATS Statement: Grading the Quality of Evidence and Strength of Recommendations in ATS Guidelines and Recommendations. Am J Respir Crit Care Med 174:605-614

12. Levy MM, Fink MP, Marshall JC, Abraham E, Angus D, Cook D, Cohen J, Opal SM, Vincent JL, Ramsay $\mathrm{G}, \mathrm{SCCM} / \mathrm{ESICM} / \mathrm{ACCP} / \mathrm{ATS} / \mathrm{SIS}$ (2003) 2001 SCCM/ESICM/ACCP/ ATS/SIS International Sepsis Definitions Conference. Crit Care Med 31:1250-1256

13. Bone RC, Balk RA, Cerra FB, Dellinger RP, Fein AM, Knaus WA, Schein RMH, Sibbald WJ, Members of the ACCP/SCCM Consensus Conference (1992) Definitions for Sepsis and Organ Failure and Guidelines for the Use of Innovative Therapies in Sepsis. Chest 101:1644-1655 and Crit Care Med 20:864-874

14. Sprung CL, Bernard GR, Dellinger RP (2001) Guidelines for the Management of Severe Sepsis and Septic Shock. Intensive Care Med 27 (Suppl 1):S1-S134

15. Sackett DL (1989) Rules of evidence and clinical recommendations on the use of antithrombotic agents. Chest 95:2S-4S

\section{Initial Resuscitation}

16. Rivers E, Nguyen B, Havstad S, Ressler J, Muzzin A, Knoblich B, Peterson E, Tomlanovich M, Early Goal-Directed Therapy Collaborative Group (2001) Early goaldirected therapy in the treatment of severe sepsis and septic shock. N Engl J Med 345:1368-1377

17. Bendjelid K, Romand JA (2003) Fluid responsiveness in mechanically ventilated patients: A review of indices used in intensive care. Intensive Care Med 29:352-360
18. Malbrain ML, Deeren D, De Potter TJ (2005) Intra-abdominal hypertension in the critically ill: It is time to pay attention. Curr Opin Crit Care 11:156-171

19. Varpula M, Tallgren M, Saukkonen K, Voipio-Pulkki LM, Pettilä V (2005) Hemodynamic variables related to outcome in septic shock. Intensive Care Med 31:1066-1071

20. Kortgen A, Niederprum P, Bauer M (2006) Implementation of an evidencebased "standard operating procedure" and outcome in septic shock. Crit Care Med 34:943-949

21. Sebat F, Johnson D, Musthafa AA, Watnik M, Moore S, Henry K, Saari M (2005) A multidisciplinary community hospital program for early and rapid resuscitation of shock in nontrauma patients. Chest 127:1729-1743

22. Shapiro NI, Howell MD, Talmor D, Lahey D, Ngo L, Buras J, Wolfe RE, Weiss JW, Lisbon A (2006) Implementation and outcomes of the Multiple Urgent Sepsis Therapies (MUST) protocol. Crit Care Med 34:1025-1032

23. Micek ST, Roubinian N, Heuring T, Bode M, Williams J, Harrison C, Murphy T, Prentice D, Ruoff BE, Kollef MH (2006) Before-after study of a standardized hospital order set for the management of septic shock. Crit Care Med 34:2707-2713

24. Nguyen HB, Corbett SW, Steele R, Banta J, Clark RT, Hayes SR, Edwards J, Cho TW, Wittlake WA (2007) Implementation of a bundle of quality indicators for the early management of severe sepsis and septic shock is associated with decreased mortality. Crit Care Med 35:1105-1112

25. Shorr AF, Micek ST, Jackson WL Jr, Kollef MH (2007) Economic implications of an evidence-based sepsis protocol: can we improve outcomes and lower costs? Crit Care Med 35:1257-1262

26. Reinhart K, Kuhn HJ, Hartog C, Bredle DL (2004) Continuous central venous and pulmonary artery oxygen saturation monitoring in the critically ill. Intensive Care Med 30:1572-1578

27. Trzeciak S, Dellinger RP, Abate NL, Cowan RM, Stauss M, Kilgannon JH, Zanotti S, Parrillo JE (2006) Translating research to clinical practice: a 1-year experience with implementing early goal-directed therapy for septic shock in the emergency department. Chest 129:225-232

28. Magder S (2006) Central venous pressure: A useful but not so simple measurement. Crit Care Med 34:2224-2227 
29. Bendjelid K (2005) Right arterial pressure: Determinant or result of change in venous return? Chest 128:3639-3640

30. Vincent JL, Weil MH (2006)

Fluid challenge revisited. Crit Care Med 34:1333-1337

31. Trzeciak S, Dellinger RP, Parrillo JE, Guglielmi M, Bajaj J, Abate NL, Arnold RC, Colilla S, Zanotti S, Hollenberg SM, Microcirculatory Alterations in Resuscitation and Shock Investigators (2007) Early microcirculatory perfusion derangements in patients with severe sepsis and septic shock: Relationship to hemodynamics, oxygen transport, and survival. Ann Emerg Med 49:88-98

32. De Backer D, Creteur J, Dubois MJ, Sakr Y, Koch M, Verdant C, Vincent JL (2006) The effects of dobutamine on microcirculatory alterations in patients with septic shock are independent of its systemic effects. Crit Care Med 34:403-408

33. Buwalda M, Ince C (2002) Opening the microcirculation: Can vasodilators be useful in sepsis? Intensive Care Med 28:1208-1217

34. Boldt J (2002) Clinical review; hemodynamic monitoring in the intensive care unit. Crit Care 6:52-59

35. Pinsky MR, Payen D (2005) Functional hemodynamic monitoring. Crit Care 9:566-572

\section{Diagnosis}

36. Weinstein MP, Reller LP, Murphy JR Lichtenstein KA (1983) The clinical significance of positive blood cultures: A comprehensive analysis of 500 episodes of bacteremia and fungemia in adults. I. Laboratory and epidemiologic observations. Rev Infect Dis 5:35-53

37. Blot F, Schmidt E, Nitenberg G, Tancrède $\mathrm{C}$, Laplanche $\mathrm{A}$, Andremont $\mathrm{A}$ (1998) Earlier positivity of central venous versus peripheral blood cultures is highly predictive of catheter-related sepsis. J Clin Microbiol 36:105-109

38. Mermel LA, Maki DG (1993) Detection of bacteremia in adults: consequences of culturing an inadequate volume of blood. Ann Intern Med 119:270-272

39. American Thoracic Society, Infectious Diseases Society of America (2005) Guidelines for the management of adults with hospital-acquired, ventilator-associated, and healthcareassociated pneumonia. Am J Respir Crit Care Med 171:388-416
40. Giamarellos-Bourboulis EJ, Giannopoulou P, Grecka P, Voros D, Mandragos K, Giamarellou H (2004) Should procalcitonin be introduced in the diagnostic criteria for the systemic inflammatory response syndrome and sepsis? J Crit Care 19:152-157

41. Tenover FC (2007) Rapid detection and identification of bacterial pathogens using novel molecular technologies: infection control and beyond. Clin Infect Dis 44:418-423

\section{Antibiotic Therapy}

42. Kumar A, Roberts D, Wood KE Light B, Parrillo JE, Sharma S, Suppes R, Feinstein D, Zanotti S, Taiberg L, Gurka D, Kumar A Cheang M (2006) Duration of hypotension prior to initiation of effective antimicrobial therapy is the critical determinant of survival in human septic shock. Crit Care Med 34:1589-1596

43. Morrell M, Fraser VJ, Kollef MH (2005) Delaying the empiric treatment of candida bloodstream infection until positive blood culture results are obtained: a potential risk factor for hospital mortality. Antimicrob Agents Chemother 49:3640-3645

44. Pappas PG, Rex JH, Sobel JD, Filler SG, Dismukes WE, Walsh TJ, Edwards JE, Infectious Diseases Society of America (2004) Guidelines for treatment of candidiasis. Clin Infect Dis 38:161-189

45. McCabe WR, Jackson GG (1962) Gram negative bacteremia. Arch Intern Med 110:92-100

46. Kreger BE, Craven DE, McCabe WR (1980) Gram negative bacteremia. IV. Re-evaluation of clinical features and treatment in 612 patients. Am J Med 68:344-355

47. Leibovici L, Shraga I, Drucker M, Konigsberger H, Samra Z, Pitlik SD (1998) The benefit of appropriate empirical antibiotic treatment in patients with bloodstream infection. J Intern Med 244:379-386

48. Ibrahim EH, Sherman G, Ward S, Fraser VJ, Kollef MH (2000) The influence of inadequate antimicrobial treatment of bloodstream infections on patient outcomes in the ICU setting. Chest 118:146-155

49. Hatala R, Dinh T, Cook DJ (1996) Once-daily aminoglycoside dosing in immunocompetent adults: A meta-analysis Ann Intern Med 124:717-725
50. Ali MZ, Goetz MB (1997) A metaanalysis of the relative efficacy and toxicity of single daily dosing versus multiple daily dosing of aminoglycosides. Clin Infec Dis 24:796-809

51. Amsden GW, Ballow CH, Bertino JS (2000) Pharmacokinetics and Pharmacodynamics of Anti-infective Agents. In: Mandell GL, Bennett JE, Dolin R (Eds) Principles and Practice of Infectious Diseases. Fifth Edition. Philadelphia, Churchill Livingstone, pp 253-261

52. Hyatt JM, McKinnon PS, Zimmer GS Schentag JJ (1995) The importance of pharmacokinetic/pharmacodynamic surrogate markers to outcomes. Focus on antibacterial agents. Clin Pharmacokinet 28:143-160

53. Hughes WT, Armstrong D, Bodey GP Bow EJ, Brown AE, Calandra T, Feld R, Pizzo PA, Rolston KVI, Shenep JL, Young LS. 2002 Guidelines for the Use of Antimicrobial Agents in Neutropenic Patients with Cancer. www.idsociety.org. Accessed July 10, 2007.

54. Klastersky J (2004) Management of fever in neutropenic patients with different risks of complications. Clin Infect Dis 39(Suppl 1):S32-37

55. Safdar N, Handelsman J, Maki DG (2004) Does combination antimicrobial therapy reduce mortality in Gram-negative bacteraemia? A meta-analysis. Lancet Infect Dis 4(8):519-527

56. Paul M, Silbiger I, Grozinsky S, Soares-Weiser K, Leibovici L (2006) Beta lactam antibiotic monotherapy versus beta lactam-aminoglycoside antibiotic combination therapy for sepsis. Cochrane Database Syst Rev Jan 25:CD003344

57. Garnacho-Montero J, Sa-Borges M, Sole-Violan J, Barcenilla F, EscorescaOrtega A, Ochoa M, Cayuela A, Rello J (2007) Optimal management therapy for Pseudomonas aeruginosa ventilator-associated pneumonia: An observational, multicenter study comparing monotherapy with combination antibiotic therapy. Crit Care Med 25:1888-1895

\section{Source Control}

58. Jimenez MF, Marshall JC (2001) Source control in the management of sepsis. Intensive Care Med 27:S49-S62

59. Moss RL, Musemeche CA, Kosloske AM (1996) Necrotizing fascitis in children: prompt recognition and aggressive therapy improve survival. J Pediatr Surg 31:1142-1146 
60. Bufalari A, Giustozzi G, Moggi L (1996) Postoperative intraabdominal abscesses: Percutaneous versus surgical treatment. Acta Chir Belg 96:197-200

61. O'Grady NP, Alexander M, Dellinger EP, Gerberding JL, Heard SO, Maki DG, Masur H, McCormick RD, Mermel LA, Pearson ML, Raad II, Randolph A, Weinstein RA (2002) Guidelines for the prevention of intravascular catheter-related infections. Centers for Disease Control and Prevention. MMWR 51(RR-10):1-29

62. O'Grady NP, Alexander M, Dellinger EP, Gerberding JL, Heard SO, Maki DG, Masur H, McCormick RD, Mermel LA, Pearson ML, Raad II, Randolph A, Weinstein RA, Healthcare Infection Control Practices Advisory Committee (2002) Guidelines for the prevention of intravascular catheter-related infections. Clin Infect Dis 35:1281-1307

63. Mier J, Leon EL, Castillo A, Robledo F, Blanco R (1997) Early versus late necrosectomy in severe necrotizing pancreatitis. Am J Surg 173:71-75

64. Evans A, Winslow BH (1995) Oxygen saturation and hemodynamic response in critically ill mechanically ventilated adults during intrahospital transport. Am J Crit Care 4:106-111

\section{Fluid Therapy}

65. Finfer S, Bellomo R, Boyce N, French J, Myburgh J, Norton R; SAFE Study Investigators (2004) A comparison of albumin and saline for fluid resuscitation in the intensive care unit. N Engl J Med 350:2247-2256

66. Choi PTL, Yip G, Quinonez LG, Cook DJ (1999) Crystalloids vs. colloids in fluid resuscitation: A systematic review. Crit Care Med 27:200-210

67. Cook D, Guyatt G (2001) Colloid use for fluid resuscitation: Evidence and spin. Ann Intern Med 135:205-208

68. Schierhout G, Roberts I (1998) Fluid resuscitation with colloid or crystalloid solutions in critically ill patients: Asystematic review of randomized trials. BMJ 316:961-964

69. Schortgen F, Lacherade JC, Bruneel F, Cattaneo I, Hemery F, Lemaire F, Brochard L (2001) Effects of hydroxyethyl starch and gelatin on renal function in severe sepsis: a multicentre randomised study. Lancet 357:911-916
70. Sakr Y, Payen D, Reinhart K, Sipmann FS, Zavala E, Bewley J, Marx G, Vincent JL (2007) Effects of hydroxyethyl starch administration on renal function in critically ill patients. Br J Anaesth 98:216-224

\section{Vasopressors}

71. Hollenberg SM, Ahrens TS, Annane D, Astiz ME, Chalfin DB, Dasta JF, Heard SO, Martin C, Napolitano LM, Susla GM, Totaro R, Vincent JL, Zanotti-Cavazzoni S (2004) Practice parameters for hemodynamic support of sepsis in adult patients: 2004 update. Crit Care Med 32:1928-1948

72. LeDoux D, Astiz ME, Carpati CM, Rackow EC (2000) Effects of perfusion pressure on tissue perfusion in septic shock. Crit Care Med 28:2729-32

73. Martin C, Papazian L, Perrin G, Saux P, Gouin F (1993) Norepinephrine or dopamine for the treatment of hyperdynamic septic shock? Chest 103:1826-1831

74. Martin C, Viviand X, Leone M, Thirion X (2000) Effect of norepinephrine on the outcome of septic shock. Crit Care Med 28:2758-2765

75. De Backer D, Creteur J, Silva E, Vincent JL (2003) Effects of dopamine, norepinephrine, and epinephrine on the splanchnic circulation in septic shock: Which is best? Crit Care Med 31:1659-1667

76. Day NP, Phu NH, Bethell DP, Mai NT, Chau TT, Hien TT, White NJ (1996) The effects of dopamine and adrenaline infusions on acid-base balance and systemic haemodynamics in severe infection. Lancet 348:219-223

77. Le Tulzo Y, Seguin P, Gacouin A, Camus C, Suprin E, Jouannic I, Thomas R (1997) Effects of epinephrine on right ventricular function in patients with severe septic shock and right ventricular failure: a preliminary descriptive study. Intensive Care Med 23:664-670

78. Bollaert PE, Bauer P, Audibert G, Lambert H, Larcan A (1990) Effects of epinephrine on hemodynamics and oxygen metabolism in dopamine-resistant septic shock. Chest 98:949-953

79. Zhou SX, Qiu HB, Huang YZ, Yang Y, Zheng RQ (2002) Effects of norepinephrine, epinephrine, and norepinephrine-dobutamine on systemic and gastric mucosal oxygenation in septic shock. Acta Pharmacologica Sinica 23:654-658
80. Levy B, Bollaert PE, Charpentier C, Nace L, Audibert G, Bauer P, Nabet P, Larcan A (1997) Comparison of norepinephrine and dobutamine to epinephrine for hemodynamics, lactate metabolism, and gastric tonometric variables in septic shock: a prospective, randomized study. Intensive Care Med 23:282-287

81. Mackenzie SJ, Kapadia F, Nimmo GR, Armstrong IR, Grant IS (1991) Adrenaline in treatment of septic shock: Effects on haemodynamics and oxygen transport. Intensive Care Med 17:36-39

82. Moran JL, O'Fathartaigh MS, Peisach AR, Chapman MJ, Leppard P (1993) Epinephrine as an inotropic agent in septic shock: a dose-profile analysis. Crit Care Med 21:70-77

83. Yamazaki T, Shimada Y, Taenaka N, Oshumi H, Takezawa J, Yoshiya I (1982) Circulatory responses to afterloading with phenylephrine in hyperdynamic sepsis. Crit Care Med 10:432-435

84. Gregory JS, Bonfiglio MF, Dasta JF, Reilley TE, Townsend MC, Flancbaum L (1991) Experience with phenylephrine as a component of the pharmacologic support of septic shock. Crit Care Med 19:1395-1400

85. Djillali A, Vigno P, Renault A, Blooaert PE, Chrpentier C, Martin C, Troché G, Ricard JD, Nitenberg G, Papazian L, Azouly E, Bellissant E, for the CATS STUDY Group (2007) Norepinephrine plus donutamine versus epinephrine alone for management of septic shock: A randomized trial. Lancet 370:676-684

86. Regnier B, Rapin M, Gory G, Lemaire F, Teisseire B, Harari A (1977) Haemodynamic effects of dopamine in septic shock. Intensive Care Med 3:47-53

87. Landry DW, Levin HR, Gallant EM, Ashton RC Jr, Seo S, D'Alessandro D, Oz MC, Oliver JA (1997) Vasopressin deficiency contributes to the vasodilation of septic shock. Circulation 95:1122-1125

88. Patel BM, Chittock DR, Russell JA, Walley KR (2002) Beneficial effects of short-term vasopressin infusion during severe septic shock. Anesthesiology 96:576-582

89. Dunser MW, Mayr AJ, Ulmer H, Knotzer H, Sumann G, Pajk W, Friesenecker B, Hasibeder WR (2003) Arginine vasopressin in advanced vasodilatory shock: a prospective, randomized, controlled study. Circulation 107:2313-2319

90. Holmes CL, Patel BM, Russell JA, Walley KR (2001) Physiology of vasopressin relevant to management of septic shock. Chest 120:989-1002 
91. Malay MB, Ashton RC, Landry DW, Townsend RN (1999) Low-dose vasopressin in the treatment of vasodilatory septic shock. J Trauma 47:699-705

92. Holmes CL, Walley KR, Chittock DR, Lehman T, Russell JA (2001) The effects of vasopressin on hemodynamics and renal function in severe septic shock: a case series. Intensive Care Med 27:1416-1421

93. Lauzier F, Levy B, Lamarre P, Lesur O (2006) Vasopressin or norepinephrine in early hyperdynamic septic shock: a randomized clinical trial. Intensive Care Med 32:1782-1789

94. O'Brien A, Calpp L, Singer M (2002) Terlipressin for norepinephrine-resistant septic shock. Lancet 359:1209-1210

95. Sharshar T, Blanchard A, Paillard M, Raphael JC, Gajdos P, Annane D (2003) Circulating vasopressin levels in septic shock. Crit Care Med 31:1752-1758

96. Dunser MW, Mayr AJ, Tur A, Pajk W, Barbara F, Knotzer H, Ulmer H, Hasibeder WR (2003) Ischemic skin lesions as a complication of continuous vasopressin infusion in catecholamine-resistant vasodilatory shock: incidence and risk factors. Crit Care Med 31:1394-1398

97. Bellomo R, Chapman M, Finfer S, Hickling K, Myburgh J (2000) Low-dose dopamine in patients with early renal dysfunction: a placebocontrolled randomised trial. Australian and New Zealand Intensive Care Society (ANZICS) Clinical Trials Group. Lancet 356:2139-2143

98. Kellum J, Decker J (2001)

Use of dopamine in acute renal failure: a meta-analysis. Crit Care Med 29:1526-1531

\section{Inotropic Therapy}

99. Gattinoni L, Brazzi L, Pelosi P, Latini R, Tognoni G, Pesenti A, Fumagalli R (1995) A trial of goal-oriented hemodynamic therapy in critically ill patients. N Engl J Med 333:1025-1032

100. Hayes MA, Timmins AC, Yau EH, Palazzo M, Hinds CJ, Watson D (1994) Elevation of systemic oxygen delivery in the treatment of critically ill patients. N Engl J Med 330:1717-1722

\section{Steroids}

101. Annane D, Sebille V, Charpentier C, Bollaert PE, François B, Korach JM, Capellier G, Cohen Y, Azoulay E, Troché G, Chaumet-Riffaut P, Bellissant E (2002) Effect of treatment with low doses of hydrocortisone and fludrocortisone on mortality in patients with septic shock. JAMA 288:862-871

102. Briegel J, Forst H, Haller M, Schelling G, Kilger E, Kuprat G, Hemmer B, Hummel T, Lenhart A, Heyduck M, Stoll C, Peter K (1999) Stress doses of hydrocortisone reverse hyperdynamic septic shock: a prospective, randomized, double-blind, single-center study. Crit Care Med 27:723-732

103. Bollaert PE, Charpentier C, Levy B, Debouverie M, Audibert G, Larcan A (1998) Reversal of late septic shock with supraphysiologic doses of hydrocortisone. Crit Care Med 26:645-650

104. Sprung CL, Annane D, Briegel J, Keh D, Moreno R, Singer M, Weiss Y, Sorenson F (2007) Corticosteroid therapy of septic shock (CORTICUS) (abstract). Am Rev Resp Crit Care Med 175:A507

105. Briegel J, Vogeser M, Annane D, Keh D, Moreno R, Singer M, Weiss Y, Sprung CL (2007) Measurement of cortisol in septic shock: Interlaboratory harmonization. Am Rev Resp Crit Care Med 175:A436

106. Allolio B, Dorr H, Stuttmann R, Knorr D, Engelhardt D, Winkelmann W (1985) Effect of a single bolus of etomidate upon eight major corticosteroid hormone and plasma ACTH. Clin Ednocrinol (oxf) 22:281-286

107. Reincke M, Allolio B, Würth G, Winkelmann W (1993) The hypothalamic-pituitary-adrenal axis in critical illness: Response to dexamethasone and corticotropinreleasing hormone. J Clin Endocrinol Metab 77:151-156

108. Oppert M, Schindler R, Husung C, Offermann K, Graf KJ, Boenisch O, Barckow D, Frei U, Eckardt KU (2005) Low dose hydrocortisone improves shock reversal and reduces cytokine levels in early hyperdynamic septic shock. Crit Care Med 33:2457-2464

109. Yildiz O, Doganay M, Aygen B, Guven M, Keleutimur F, Tutuu A (2002) Physiologic-dose steroid therapy in sepsis. Crit Care 6:251-259
110. Keh D, Boehnke T, Weber-Cartens S, Schulz C, Ahlers O, Bercker S, Volk HD, Doecke WD, Falke KJ, Gerlach H (2003) Immunologic and hemodynamic effects of "low-dose" hydrocortisone in septic shock: a double-blind, randomized, placebocontrolled, crossover study. Am J Respir Crit Care Med 167:512-520

111. Bone RC, Fisher CJ, Clemmer TP (1987) A controlled clinical trial of high-dose methylprednisolone in the treatment of severe sepsis and septic shock. N Engl J Med 317:653-658

112. Cronin L, Cook DJ, Carlet J, Heyland DK, King D, Lansang MA, Fisher CJ Jr (1995) Corticosteroid treatment for sepsis: a critical appraisal and meta-analysis of the literature. Crit Care Med 23:1430-1439

113. The Veterans Administration Systemic Sepsis Cooperative Study Group (1987) Effect on high-dose glucocorticoid therapy on mortality in patients with clinical signs of sepsis. N Engl J Med 317:659-665

114. Confalonieri M, Urbino R, Potena A, Piattella M, Parigi P, Puccio G, Della Porta R, Giorgio C, Blasi F, Umberger R, Meduri GU (2005) Hydrocortisone infusion for severe community-acquired pneumonia. A preliminary randomized study. Am J Respir Crit Care Med 171:242-248

Recombinant Human Activated Protein C

115. Bernard GR, Vincent JL, Laterre PF, LaRosa SP, Dhainaut JF, Lopez-Rodriguez A, Steingrub JS, Garber GE, Helterbrand JD, Ely EW, Fisher CJ Jr; Recombinant human protein $\mathrm{C}$ Worldwide Evaluation in Severe Sepsis (PROWESS) study group (2001) Efficacy and safety of recombinant human activated protein $\mathrm{c}$ for severe sepsis. N Engl J Med 344:699-709

116. Abraham E, Laterre PF, Garg R, Levy H, Talwar D, Trzaskoma BL, Francois B, Guy JS, Bruckmann M, Rea-Neto A, Rossaint R, Perrotin D, Sablotzki A, Arkins N, Utterback BG, Macias WL, Administration of Drotrecogin Alfa (Activated) in Early Stage Severe Sepsis (ADDRESS) Study Group (2005) Drotrecogin alfa (activated) for adults with severe sepsis and a low risk of death. N Engl J Med 353:1332-1341 
117. Vincent JL, Bernard GR, Beale R, Doig C, Putensen C, Dhainaut JF, Artigas A, Fumagalli R, Macias W, Wright T, Wong K, Sundin DP, Turlo MA, Janes J (2005) Drotrecogin alfa (activated) treatment in severe sepsis from the global openlabel trial ENHANCE: Further evidence for survival and safety and implications for early treatment. Crit Care Med 33:2266-2277

118. Oxman AD, Guyatt GH (1992) A consumer's guide to subgroup analyses. Ann Intern Med 116:78-84

119. Ely EW, Laterre PF, Angus DC, Helterbrand JD, Levy H, Dhainaut JF, Vincent JL, Macias WL, Bernard GR (2003) Drotrecogin alfa (activated) administration across clinically important subgroups of patients with severe sepsis. Crit Care Med 31:12-19

120. Kanji S, Perreault MM, Chant C, Williamson D, Burry L (2007) Evaluating the use of Drotrecogin alfa activated in adult severe sepsis: a Canadian multicenter observational study. Intensive Care Med 33:517-523

121. Bertolini G, Rossi C, Anghileri A, Livigni S, Addis A, Poole D (2007) Use of Drotrecogin alfa (activated) in Italian intensive care units: The results of a nationwide survey. Intensive Care Med 33:426-434

122. European Medicines Agency. Evaluation of Medicines for Human Use. www.emea.europa.eu/pdfs/human/ press/pr/8509607en.pdf.

Accessed October 22, 2007.

\section{Blood Product Administration}

123. Hebert PC, Wells G, Blajchman MA, Marshall J, Martin C, Pagliarello G, Tweeddale M, Schweitzer I, Yetisir E (1999) A multicenter, randomized, controlled clinical trial of transfusion in critical care. N Engl J Med 340:409-417

124. Marik PE, Sibbald WJ (1993) Effect of stored-blood transfusion on oxygen delivery in patients with sepsis. JAMA 269:3024-3029

125. Lorente JA, Landín L, dePablo R, Renes E, Rodriguez-Diaz R, Liste D (1993) Effects of blood transfusion on oxygen transport variables in severe sepsis. Crit Care Med 21:1312-1318

126. Fernandes CJ Jr, Akamine N, De Marco FV, De Souza JA, Lagudis S, Knobel E (2001) Red blood cell transfusion does not increase oxygen consumption in critically ill septic patients. Crit Care 5:362-567
127. Corwin HL, Gettinger A, Rodriguez RM, Pearl RG, Gubler KD, Enny C, Colton T, Corwin MJ (1999) Efficacy of recombinant human erythropoietin in the critically ill patient: a randomized double-blind, placebo-controlled trial. Crit Care Med 27:2346-2350

128. Corwin HL, Gettinger A, Pearl RG, Fink MP, Levy MM, Shapiro MJ, Corwin MJ, Colton T, EPO Critical Care Trials Group (2002) Efficacy of recombinant human erythropoietin in critically ill patients. JAMA 28:2827-2835

129. College of American Pathologists (1994) Practice parameter for the use of fresh-frozen plasma, cryoprecipitate, and platelets. JAMA 271:777-781

130. Canadian Medical Association Expert Working Group (1997) Guidelines for red blood cell and plasma transfusion for adults and children. Can Med Assoc J 156:S1-S24

131. American Society of Anaesthesiologists Task Force on Blood Component Therapy (1996) Practice guidelines for blood component therapy. Anesthesiology 84:732-47

132. Abdel-Wahab OI, Healy B, Dzik WH (2006) Effect of fresh-frozen plasma transfusion on prothrombin time and bleeding in patients with mild coagulation abnormalities. Transfusion 46:1279-85

133. Warren BL, Eid A, Singer P, Pillay SS, Carl P, Novak I, Chalupa P, Atherstone A, Penzes I, Kubler A, Knaub S, Keinecke HO, Heinrichs H, Schindel F, Juers M, Bone RC, Opal SM, KyberSept Trial Study Group (2001) High-dose antithrombin III in severe sepsis. A randomized controlled trial. JAMA 286:1869-1878

134. Wiedermann CJ, Hoffmann JN, Juers M, Ostermann H, Kienast J, Briegel J, Strauss R, Keinecke HO, Warren BL, Opal SM, KyberSept Investigators (2006) High-dose antithrombin III in the treatment of severe sepsis in patients with a high risk of death: Efficacy and safety. Crit Care Med 34:285-292

\section{Mechanical Ventilation}

135. The Acute Respiratory Distress Syndrome Network (2000) Ventilation with lower tidal volumes as compared with traditional tidal volumes for acute lung injury and the acute respiratory distress syndrome. N Engl J Med 342:1301-1308
136. Amato MB, Barbas CS, Medeiros DM, Magaldi RB, Schettino GP, LorenziFilho G, Kairalla RA, Deheinzelin D, Munoz C, Oliveira R, Takagaki TY, Carvalho CR (1998) Effect of a protective-ventilation strategy on mortality in the acute respiratory distress syndrome. N Engl J Med 338:347-354

137. Brochard L, Roudot-Thoraval F, Roupie E, Delclaux C, Chastre J, Fernandez-Mondejar E, Clementi E, Mancebo J, Factor P, Matamis D, Ranieri M, Blanch L, Rodi G, Mentec H, Dreyfuss D, Ferrer M, Brun-Buisson C, Tobin M, Lemaire F (1998) Tidal volume reduction for prevention of ventilator-induced lung injury in acute respiratory distress syndrome. Am J Respir Crit Care Med 158:1831-1838

138. Brower RG, Shanholtz CB, Fessler HE, Shade DM, White P Jr, Wiener CM, Teeter JG, Dodd-o JM, Almog Y, Piantadosi S (1999) Prospective, randomized, controlled clinical trial comparing traditional versus reduced tidal volume ventilation in acute respiratory distress syndrome patients. Crit Care Med 27:1492-1498

139. Stewart TE, Meade MO, Cook DJ, Granton JT, Hodder RV, Lapinsky SE, Mazer CD, McLean RF, Rogovein TS, Schouten BD, Todd TR, Slutsky AS (1998) Evaluation of a ventilation strategy to prevent barotrauma in patients at high risk for acute respiratory distress syndrome. N Engl J Med 338:355-361

140. Eichacker PQ, Gerstenberger EP Banks SM, Cui X, Natanson C (2002) Meta-analysis of acute lung injury and acute respiratory distress syndrome trials testing low tidal volumes. Am J Respir Crit Care Med 166:1510-1514

141. Tobin MJ (2000) Culmination of an era in research on the acute respiratory distress syndrome. N Engl J Med 342:1360-1361

142. Marini JJ, Gattinoni L (2004) Ventilatory management of acute respiratory distress syndrome: A consensus of two. Crit Care Med 32:250-255

143. Hager DN, Krishnan JA, Hayden DL, Brower RG; ARDS Clinical Trials Network (2005) Tidal volume reduction in patients with acute lung injury when plateau pressures are not high. Am J Respir Crit Care Med 172:1241-1245 
144. Ferguson ND, Frutos-Vivar F, Esteban A, Anzueto A, Alia I, Brower RG, Stewart TE, Apezteguia C, Gonzalez M, Soto L, Abroug F, Brochard L, Mechanical Ventilation International Study Group (2005) Airway pressures, tidal volumes, and mortality in patients with acute respiratory distress syndrome Crit Care Med 33:21-30

145. Hickling KG, Henderson S, Jackson R (1994) Low mortality rate in adult respiratory distress syndrome using low-volume, pressure-limited ventilation with permissive hypercapnia: a prospective study. Crit Care Med 22:1568-1578

146. Bidani A, Tzouanakis AE, Cardenas VJ Jr, Zwischenberger JB (1994) Permissive hypercapnia in acute respiratory failure. JAMA 272:957-962

147. Kallet RH, Jasmer RM, Luce JM, Lin LH, Marks JD (2000) The treatment of acidosis in acute lung injury with tris-hydroxymethyl aminomethane (THAM). Am J Respir Crit Care Med 161(4 Pt 1):1149-1153

148. Weber T, Tschernich H, Sitzwohl C, Ullrich R, Germann P, Zimpfer M, Sladen RN, Huemer G (2000) Tromethamine buffer modifies the depressant effect of permissive hypercapnia on myocardial contractility in patients with acute respiratory distress syndrome. Am J Respir Crit Care Med 162:1361-1365

149. Marini JJ, Ravenscraft SA (1992) Mean airway pressure: physiologic determinants and clinical importance-Part 1: Physiologic determinants and measurements. Crit Care Med 20:1461-1472

150. Gattinoni L, Marcolin R, Caspani ML, Fumagalli R, Mascheroni D, Pesenti A (1985) Constant mean airway pressure with different patterns of positive pressure breathing during the adult respiratory distress syndrome. Bull Eur Physiopathol Respir 21:275-279

151. Pesenti A, Marcolin R, Prato P, Borelli M, Riboni A, Gattinoni L (1985) Mean airway pressure vs. positive end-expiratory pressure during mechanical ventilation. Crit Care Med 13:34-37

152. The National Heart, Lung, and Blood Institute ARDS Clinical Trials Network (2004) Higher versus lower positive end-expiratory pressures in patients with the acute respiratory distress syndrome. N Engl J Med 351:327-336

153. Villar J, Kacmarek RM, Pérez-Méndez L, Aguirre-Jaime A, for the ARIES Network (2006) A high PEEP-low tidal volume ventilatory strategy improves outcome in persistent ARDS. A randomized controlled trial. Crit Care Med 34:1311-1318
154. Amato MB, Barbas CS, Medeiros DM, Schettino Gde P, Lorenzi Filho G, Kairalla RA, Deheinzelin D, Morais C, Fernandes Ede O, Takagaki TY, et al. (1995) Beneficial effects of the "open lung approach" with low distending pressures in acute respiratory distress syndrome. A prospective randomized study on mechanical ventilation. Am J Respir Crit Care Med 152:1835-1846

155. Gattinoni L, Caironi P, Cressoni M, Chiumello D, Ranieri VM, Quintel M, Russo S, Patroniti N, Cornejo R, Bugedo G (2006) Lung recruitment in patients with acute respiratory distress syndrome. N Engl J Med 354:1775-1786

156. Stocker R, Neff T, Stein S, Ecknauer E, Trentz O, Russi E (1997) Prone positioning and low-volume pressure-limited ventilation improve survival in patients with severe ARDS. Chest 111:1008-1017

157. Lamm WJ, Graham MM, Albert RK (1994) Mechanism by which prone position improves oxygenation in acute lung injury. Am J Respir Crit Care Med 150:184-193

158. Jolliet P, Bulpa P, Chevrolet JC (1998) Effects of the prone position on gas exchange and hemodynamics in severe acute respiratory distress syndrome. Crit Care Med 26:1977-1985

159. Gattinoni L, Tognoni G, Pesenti A, Taccone P, Mascheroni D, Labarta V, Malacrida R, Di Giulio P, Fumagalli R, Pelosi P, Brazzi L, Latini R, ProneSupine Study Group (2001) Effect of prone positioning on the survival of patients with acute respiratory failure. N Engl J Med 345:568-573

160. Guerin C, Gaillard S, Lemasson S, Ayzac L, Girard R, Beuret P, Palmier B, Le QV, Sirodot M, Rosselli S, Cadiergue V, Sainty JM, Barbe P, Combourieu E, Debatty D, Rouffineau J, Ezingeard E, Millet O, Guelon D, Rodriguez L, Martin O, Renault A, Sibille JP, Kaidomar M (2004) Effects of systematic prone positioning in hypoxemic acute respiratory failure: a randomized controlled trial. JAMA 292:2379-2387

161. Mancebo J, Fernández R, Blanch L, Rialp G, Gordo F, Ferrer M, Rodríguez F, Garro P, Ricart P, Vallverdú I, Gich I, Castaño J, Saura P, Domínguez G, Bonet A, Albert RK (2006) A multicenter trial of prolonged prone ventilation in severe acute respiratory distress syndrome. Am J Respir Crit Care Med 173:1233-1239
162. Drakulovic MB, Torres A, Bauer TT, Nicolas JM, Nogue S, Ferrer M (1999) Supine body position as a risk factor for nosocomial pneumonia in mechanically ventilated patients: a randomised trial. Lancet 354:1851-1858

163. van Nieuwenhoven CA, Vandenbroucke-Grauls C, van Tiel FH, Joore HC, van Schijndel RJ, van der Tweel I, Ramsay G, Bonten MJ (2006) Feasibility and effects of the semirecumbent position to prevent ventilator-associated pneumonia: a randomized study. Crit Care Med 34:396-402

164. Antonelli M, Conti G, Rocco M, Bufi M, De Blasi RA, Vivino G, Gasparetto A, Meduri GU (1998) A comparison of noninvasive positive-pressure ventilation and conventional mechanical ventilation in patients with acute respiratory failure. N Engl J Med 339:429-435

165. Ferrer M, Esquinas A, Leon M, Gonzalez G, Alarcon A, Torres A (2003) Noninvasive ventilation in severe hypoxemic respiratory failure: a randomized clinical trial. Am J Resp Crit Care Med 168:1438-1444

166. Ely EW, Baker AM, Dunagan DP, Burke HL, Smith AC, Kelly PT, Johnson MM, Browder RW, Bowton DL, Haponik EF (1996) Effect on the duration of mechanical ventilation of identifying patients capable of breathing spontaneously. N Engl J Med 335:1864-1869

167. Esteban A, Alía I, Tobin MJ, Gil A, Gordo F, Vallverdú I, Blanch L, Bonet A, Vázquez A, de Pablo R, Torres A, de La Cal MA, Macías S (1999) Effect of spontaneous breathing trial duration on outcome of attempts to discontinue mechanical ventilation. Am J Respir Crit Care Med 159:512-518

168. Esteban A, Alia I, Gordo F, Fernandez R, Solsona JF, Vallverdu I, Macias S, Allegue JM, Blanco J, Carriedo D, Leon M, de la Cal MA, Taboada F, Gonzalez de Velasco J, Palazon E, Carrizosa F, Tomas R, Suarez J, Goldwasser RS (1997) Extubation outcome after spontaneous breathing trials with T-tube or pressure support ventilation. Am J Respir Crit Care Med 156:459-465

169. Brochard L, Rauss A, Benito S Conti G, Mancebo J, Rekik N, Gasparetto A, Lemaire F (1994) Comparison of three methods of gradual withdrawal from ventilatory support during weaning from mechanical ventilation. Am J Respir Crit Care Med 150:896-903 
170. Connors AF Jr, McCaffree DR, Gray BA (1983) Evaluation of rightheart catheterization in the critically ill patient without acute myocardial infarction. N Engl J Med 308:263-267

171. Iberti TJ, Fischer EP, Leibowitz AB, Panacek EA, Silverstein JH, Albertson TE (1990) A multicenter study of physicians' knowledge of the pulmonary artery catheter. Pulmonary artery catheter study group. JAMA 264:2928-2932

172. Al-Kharrat T, Zarich S, AmoatengAdjepong Y, Manthous CA (1999) Analysis of observer variability in measurement of pulmonary artery occlusion pressures. Am J Respir Crit Care Med 160:415-420

173. Osman D, Ridel C, Ray P, Monnet X, Anguel N, Richard C, Teboul JL (2007) Cardiac filling pressures are not appropriate to predict hemodynamic response to volume challenge. Crit Care Med 35:64-68

174. Gattinoni L, Brazzi L, Pelosi P, Latini R, Tognoni G, Pesenti A, Fumagalli R (1995) A trial of goal-oriented hemodynamic therapy in critically ill patients: $\mathrm{SvO} 2$ collaborative group. N Engl J Med 333:1025-1032

175. Richard C, Warszawski J, Anguel N, Deye N, Combes A, Barnoud D, Boulain T, Lefort Y, Fartoukh M, Baud F, Boyer A, Brochard L, Teboul JL, French Pulmonary Artery Catheter Study Group (2003) Early use of the pulmonary artery catheter and outcomes in patients with shock and acute respiratory distress syndrome: A randomized controlled trial. JAMA 290:2713-2720

176. National Heart, Lung and Blood Institute Acute Respiratory Distress Syndrome (ARDS) Clinical Trials Network, Wheeler AP, Bernard GR, Thompson BT, Schoenfeld D, Wiedemann HP, deBoisblanc B, Connors AF Jr, Hite RD, Harabin AL (2006) Pulmonary-artery versus central venous catheter to guide treatment of acute lung injury. N Engl J Med 354:2213-2224

177. Sandham JD, Hull RD, Brant RF, Knox L, Pineo GF, Doig CJ, Laporta DP, Viner S, Passerini L, Devitt H, Kirby A, Jacka M, Canadian Critical Care Clinical Trials Group (2003) A randomized, controlled trial of the use of pulmonary-artery catheters in high-risk surgical patients. N Engl J Med 348:5-14

178. Shah MR, Hasselblad V, Stevenson LW, Binanay C, O'Connor CM, Sopko G, Califf RM (2005) Impact of the pulmonary artery catheter in critically ill patients: Metaanalysis of randomized clinical trials. JAMA 294:1664-1670
179. Harvey S, Harrison DA, Singer M, Ashcroft J, Jones CM, Elbourne D, Brampton W, Williams D, Young D, Rowan K, PAC-Man study collaboration (2005) Assessment of the clinical effectiveness of pulmonary artery catheters in management of patients in intensive care (PACman): A randomised controlled trial. Lancet 366:472-477

180. Ware LB, Matthay MA (2000) The acute respiratory distress syndrome. N Engl J Med 342:1334-1349

181. Sibbald WJ, Short AK, Warshawski FJ, Cunningham DG, Cheung H (1985) Thermal dye measurements of extravascular lung water in critically ill patients: intravascular starling forces and extravascular lung water in the adult respiratory distress syndrome. Chest 87:585-592

182. Martin GS, Mangialardi RJ, Wheeler AP, Dupont WD, Morris JA, Bernard GR (2002) Albumin and furosemide therapy in hypoproteinemic patients with acute lung injury. Crit Care Med 30:2175-2182

183. Schuller D, Mitchell JP, Calandrino FS, Schuster DP (1991) Fluid balance during pulmonary edema: Is fluid again a marker or a cause of poor outcome? Chest 100:1068-1075

184. Mitchell JP, Schuller D, Calandrino FS, Schuster DP (1992) Improved outcome based on fluid management in critically ill patients requiring pulmonary artery catheterization. Am Rev Respir Dis 145:990-998

185. National Heart, Lung and Blood Institute Acute Respiratory Distress Syndrome (ARDS) Clinical Trials Network, Wiedemann HP, Wheeler AP, Bernard GR, Thompson BT, Hayden D, de Boisblanc B, Connors AF Jr, Hite RD, Harabin AL (2006) Comparison of two fluid-management strategies in acute lung injury. N Engl J Med 354:2564-2575

Sedation, Analgesia and

Neuromuscular Blockade

186. Brook AD, Ahrens TS, Schaiff R, Prentice D, Sherman G, Shannon W, Kollef MH (1999) Effect of a nursingimplemented sedation protocol on the duration of mechanical ventilation. Crit Care Med 27:2609-2615

187. Marx WH, DeMaintenon NL Mooney KF, Mascia ML, Medicis J, Franklin PD, Sivak E, Rotello L (1999) Cost reduction and outcome improvement in the intensive care unit. J Trauma 46:625-629
188. MacLaren R, Plamondon JM, Ramsay KB, Rocker GM, Patrick WD, Hall RI (2000) A prospective evaluation of empiric versus protocolbased sedation and analgesia. Pharmacotherapy 20:662-672

189. De Jonghe B, Cook D, AppereDe-Vecchi C, Guyatt G, Meade M, Outin H (2000) Using and understanding sedation scoring systems: a systematic review. Intensive Care Med 20:662-672

190. Devlin JW, Boleski G, Mlynarek M, Nerenz DR, Peterson E, Jankowski M, Horst HM, Zarowitz BJ (1999) Motor activity assessment scale: A valid and reliable sedation scale for use with mechanically ventilated patients in an adult surgical intensive care unit. Crit Care Med 27:1271-1275

191. Kollef MH, Levy NT, Ahrens TS, Schaiff R, Prentice D, Sherman G (1998) The use of continuous IV sedation is associated with prolongation of mechanical ventilation. Chest 114:541-548

192. Kress JP, Pohlman AS, O'Connor MF, Hall JB (2000) Daily interruption of sedative infusions in critically ill patients undergoing mechanical ventilation. N Engl J Med 342:1471-1477

193. Kress JP, Vinayak AG, Levitt J, Schweickert WD, Gehlbach BK, Zimmerman F, Pohlman AS, Hall JB (2007) Daily sedative interruption in mechanically ventilated patients at risk for coronory disease. Crit Care Med 35:365-371

194. Klessig HT, Geiger HJ, Murray MJ, Coursin DB (1992) A national survey on the practice patterns of anesthesiologist intensivists in the use of muscle relaxants. Crit Care Med 20:1341-1345

195. Murray MJ, Cowen J, DeBlock H, Erstad B, Gray AW Jr, Tescher AN, McGee WT, Prielipp RC, Susla G, Jacobi J, Nasraway SA Jr, Lumb PD, Task Force of the American College of Critical Care Medicine (ACCM) of the Society of Critical Care Medicine (SCCM), American Society of Health-System Pharmacists, American College of Chest Physicians (2002) Clinical practice guidelines for sustained neuromuscular blockade in the critically ill adult. Crit Care Med 30:142-156

196. Hansen-Flaschen JH, Brazinsky S, Basile C, Lanken PN (1991) The use of sedating drugs and neuromuscular blocking agents in patients requiring mechanical ventilation for respiratory failure. JAMA 266:2870-2875 
197. Freebairn RC, Derrick J, Gomersall CD, Young RJ, Joynt GM (1997) Oxygen delivery, oxygen consumption, and gastric intramucosal $\mathrm{pH}$ are not improved by a computercontrolled, closed-loop, vecuronium infusion in severe sepsis and septic shock. Crit Care Medicine 25:72-77

198. Shapiro BA, Warren J, Egol AB, Greenbaum DM, Jacobi J, Nasraway SA, Schein RM, Spevetz A, Stone JR (1995) Practice parameters for sustained neuromuscular blockade in the adult critically ill patient: An executive summary. Crit Care Med 23:1601-1605

199. Meyer KC, Prielipp RC, Grossman JE, Coursin DB (1994) Prolonged weakness after infusion of atracurium in tow intensive care unit patients. Anesth Analg 78:772-774

200. Lacomis D, Petrella JT, Giuliani MJ (1998) Causes of neuromuscular weakness in the intensive care unit: A study of ninety-two patients. Muscle Nerve 21:610-617

201. Gooch JL, Suchyta MR, Balbierz JM, Petajan JH, Clemmer TP (1991) Prolonged paralysis after treatment with neuromuscular blocking agents. Crit Care Med 19:1125-1131

202. Rudis MI, Sikora CA, Angus E, Peterson E, Popovich J Jr, Hyzy R, Zarowitz BJ (1997) A prospective randomized controlled evaluation of peripheral nerve stimulation versus standard clinical dosing of neuromuscular blocking agents in critically ill patients. Crit Care Med 25:575-583

203. Frankel H, Jeng J, Tilly E, St Andre A, Champion H (1996) The impact of implementation of neuromuscular blockade monitoring standards in a surgical intensive care unit. Am Surg 62:503-506

204. Strange C, Vaughan L, Franklin C, Johnson J (1997) comparison of trainof-four and best clinical assessment during continuous paralysis. Am J Respir Crit Care Med 156:1556-1561

\section{Glucose Control}

205. van den Berghe G, Wouters P, Weekers F, Verwaest C, Bruyninckx F, Schetz M, Vlasselaers D, Ferdinande P, Lauwers P, Bouillon R (2001) Intensive Insulin Therapy in Critically Ill Patients. N Engl J Med 345:1359-1367
206. Van den Berghe G, Wilmer A, Hermans G, Meersseman W, Wouters PJ, Milants I, Van Wijngaerden E, Bobbaers H, Bouillon R (2006) Intensive Insulin Therapy in the Medical ICU. N Engl J Med 354:449-461

207. Krinsley JS (2004) Effect of an Intensive Glucose Management Protocol on the Mortality of Critically Ill Adult Patients. Mayo Clin Proc 79:992-1000

208. Finney SJ, Zekveld C, Elia A, Evans TW (2003) Glucose Control and Mortality in Critically Ill Patients. JAMA 290:2041-2047

209. Krinsley JS (2003) Association Between Hyperglycemia and Increased Hospital Mortality in a Heterogeneous Population of Critically Ill Patients. Mayo Clin Proc 78:1471-1478

210. Egi M, Bellomo R, Stachowski E, French C (2006) Variability in blood glucose concentrations and short-term mortality in critically ill patients. Anesthesiology 105:233-234

211. Pittas AG, Siegel RD, Lau J (2004) Insulin therapy for critically ill hospitalized patients. Arch Int Med 164:2005-2011

212. Brunkhorst FM, Kuhnt E, Engel C, Meier-Hellmann A, Ragaller M, Quintel M, Weiler N, Grundling M, Oppert M, Deufel T, et al. (2005) Intensive insulin therapy in patient with severe sepsis and septic shock is associated with an increased rate of hypoglycemia - results from a randomized multicenter study (VISEP) [abstract]. Infection 33:19-20

213. Preiser JC (2007) Intensive glycemic control in med-surg patients (European Glucontrol trial). Program and abstracts of the Society of Critical Care Medicine 36th Critical Care Congress; February 17-21, 2007; Orlando, Florida

214. Current Controlled Trials. A multicentre, open label, randomised controlled trial of two target ranges for glycaemic control in intensive care unit (ICU) patients. Available from http://controlled-trials.com/isrctn/trial/ ISRCTN04968275/0/04968275.html [accessed June 10, 2007].

215. Nichols JH (2002) Bedside testing, glucose monitoring, and diabetes management. In: Kost GJ, Principles of Point of Care testing. Lippincott Williams \& Wilkins, Philadelphia

216. Kanji S, Buffie J, Hutton B, Bunting PS, Singh A, McDonald K, Fergusson D, McIntyre LA, Hebert PC (2005) Reliability of point-of-care testing for glucose measurement in critically ill adults. Crit Care Med 33:2778-2785
217. Wilson M, Weinreb J, Soo Hoo GW (2007) Intensive insulin therapy in critical care: A review of a dozen protocols. Diabetes Care 30:1005-1011

\section{Renal Replacement}

218. Mauritz W, Sporn P, Schindler I, Zadrobilek E, Roth E, Appel W (1986) Acute renal failure in abdominal infection. Comparison of hemodialysis and continuous arteriovenous and continuous hemofiltration. Anasth Intensivther Nortfallmed 21:212-217

219. Bartlett RH, Mault JR, Dechert RE, Palmer J, Swartz RD, Port FK (1986) Continuous arteriovenous hemofiltration: improved survival in surgical acute renal failure. Surgery 100:400-408

220. Kierdorf H (1991) Continuous versus intermittent treatment: clinical results in acute renal failure. Contrib Nephrol 93:1-12

221. Bellomo R, Mansfield D, Rumble S, Shapiro J, Parkin G, Boyce N (1992) Acute renal failure in critical illness: conventional dialysis versus continuous hemodiafiltration. Am Soc Artif Intern Organs J 38:M654-M657

222. Bellomo R, Farmer M, Parkin G, Wright C, Boyce N (1995) Severe acute renal failure: a comparison of acute continuous hemodilafiltration and conventional dialytic therapy. Nephron 71:59-64

223. Kruczinski K, Irvine-Bird K, Toffelmire EB, Morton AR (1993) A comparison of continuous arteriovenous hemofiltration and intermittent hemodialysis in acute renal failure patients in intensive care unit. Am Soc Artif Intern Organs J 38:M778-M781

224. van Bommel EF, Bouvy ND, So KL, Vincent HH, Zietse R, Bruining HA, Weimar W (1995) Acute dialytic support for the critically ill : intermittent hemodialysis versus continuous arteriovenous hemodialfiltration. Am J Nephrol 15:192-200

225. Guerin C, Girard R, Selli JM, Ayzac L (2002) Intermittent versus continuous renal replacement therapy for acute renal failure in intensive care units: results from a multicenter prospective epidemiological survey. Intensive Care Med 28:1411-1418

226. Kellum JA, Angus DC, Johnson JP, Leblanc M, Griffin M, Ramakrishnan N, Linde-Zwirble WT (2002) Continuous versus intermittent renal replacement therapy: a meta-analysis. Intensive Care Med 28:29-37 
227. Tonelli M, Manns B, Feller-Kopman D (2002) Acute renal failure in the intensive care unit : a systematic review of the impact of dialytic modality on mortality and renal recovery. Am J Kidney Dis 40:875-885

228. Mehta RL, McDonald B, Gabbai FB, Pahl M, Pascual MT, Farkas A, Kaplan RM, Collaborative Group for Treatment of ARF in the ICU (2001) A randomized clinical trial of continuous versus intermittent dialysis for acute renal failure. Kidney Int 60:1154-1163

229. Gasparovic V, Filipovic-Greie I, Merkler M, Pisl Z (2003) Continuous renal replacement therapy (CRRT) or intermittent hemodialysis (IHD) - What is the procedure of choice in critically ill patients? Ren Fail 25:855-862

230. Augustine JJ, Sandy D, Seifert TH, Paganini EP (2004) A randomized controlled trial comparing intermittent with continuous dialysis in patients with ARF. Am J Kidney Dis 44:1000-1007

231. Uehlinger DE, Jakob SM, Ferrari P, Eichelberger M, Huynh-Do U, Marti HP, Mohaupt MG, Vogt B, Rothen HU, Regli B, Takala J, Frey FJ (2005) Comparison of continuous and intermittent renal replacement therapy for acute renal failure. Nephrol Dial Transplant 20:1630-1637

232. Vinsonneau C, Camus C, Combes A, Costa de Beauregard MA, Klouche K, Boulain T, Pallot JL, Chiche JD, Taupin P, Landais P, Dhainaut JF, Hemodiafe Study Group (2006) Continuous venovenous haemodiafiltration versus intermittent haemodialysis for acute renal failure in patients with multiple-organ dysfunction syndrome: a multicentre randomised trial. Lancet 368:379-385

233. John S, Griesbach D, Baumgärtel M, Weihprecht H, Schmieder RE, Geiger H (2001) Effects of continuous haemofiltration vs intermittent heamodialysis on systemic heamodynamics and splanchnic regional perfusion in septic shock patients: a prospective, randomized clinical trial. Nephrol Dial Transplant 16:320-327

234. Misset B, Timsit JF, Chevret S, Renaud B, Tamion F, Carlet J (1996) A randomized cross-over comparison of the hemodynamic response to intermittent hemodialysis and continuous hemofiltration in ICU patients with acute renal failure. Intensive Care Med 22:742-746
235. Ronco C, Bellomo R, Homel P, Brendolan A, Dan M, Piccinni P, La Greca G (2000) Effects of different doses in continuous veno-venous haemofiltration on outcomes of acute renal failure: A prospective randomised trial. Lancet 356:26-30

236. Bouman CS, Oudemans-Van Straaten HM, Tijssen JG, Zandstra DF, Kesecioglu J (2002) Effects of early high-volume continuous venovenous hemofiltration on survival and recovery of renal function in intensive care patients with acute renal failure: A prospective, randomized trial. Crit Care Med 30:2205-2211

237. Schiffl H, Lang SM, Fischer R (2002) Daily hemodialysis and the outcome of acute renal failure. N Engl J Med 346:305-310

238. Saudan P, Niederberger M, De Seigneux S, Romand J, Pugin J, Perneger T, Martin PY (2006) Adding a dialysis dose to continuous hemofiltration increases survival in patients with acute renal failure. Kidney Int 70:1312-1317

\section{Bicarbonate Therapy}

239. Cooper DJ, Walley KR, Wiggs BR, Russell JA (1990) Bicarbonate does not improve hemodynamics in critically ill patients who have lactic acidosis: a prospective, controlled clinical study. Ann Intern Med 112:492-498

240. Mathieu D, Neviere R, Billard V, Fleyfel M, Wattel F (1991) Effects of bicarbonate therapy on hemodynamics and tissue oxygenation in patients with lactic acidosis: a prospective, controlled clinical study. Crit Care Med 19:1352-1356

\section{DVT Prophylaxis}

241. Cade JF (1982) High risk of the critically ill for venous thromboembolism. Crit Care Med 10:448-450

242. Pingleton SK, Bone RC, Pingleton WW, Ruth WE (1981) Prevention of pulmonary emboli in a respiratory intensive care unit. Chest 79:647-650

243. Halkin H, Goldberg J, Modan M, Modan B (1982) Reduction of mortality in general medical in-patients by low-dose heparin prophylaxis. Ann Intern Med 96:561-565

244. Belch JJ, Lowe GD, Ward AG, Forbes CD, Prentice CR (1981) Prevention of deep vein thrombosis in medical patients by low-dose heparin. Scott Med J 26:115-117
245. Gardlund B (1996) Randomized, controlled trial of low-dose heparin for prevention of fatal pulmonary embolism in patients with infectious diseases: The Heparin Prophylaxis Study Group. Lancet 347:1357-1361

246. Samama MM, Cohen AT, Darmon JY, Desjardins L, Eldor A, Janbon C, Leizorovicz A, Nguyen H, Olsson CG, Turpie AG, Weisslinger N (1999) A comparison of enoxaparin with placebo for the prevention of venous thromboembolism in acutely ill medical patient. N Engl J Med 341:793-800

247. Dahan R, Houlbert D, Caulin C, Cuzin E, Viltart C, Woler M, Segrestaa JM (1986) Prevention of deep vein thrombosis in elderly medical in-patients by a low molecular weight heparin: A randomized double-blind trial. Haemostasis 16:159-164

248. Hirsch DR, Ingenito EP, Goldhaber SZ (1995) Prevalence of deep venous thrombosis among patients in medical intensive care. JAMA 274:335-337

249. Fraisse F, Holzapfel L, Couland JM, Simonneau G, Bedock B, Feissel M, Herbecq P, Pordes R, Poussel JF, Roux L (2000) Nadroparin in the prevention of deep vein thrombosis in acute decompensated COPD: The Association of Non-University Affiliated Intensive Care Specialist Physicians of France. Am J Respir Crit Care Med 161:1109-1114

250. Kupfer Y, Anwar J, Seneviratne C, et al. (1999) Prophylaxis with subcutaneous heparin significantly reduces the incidence of deep venous thrombophlebitis in the critically ill. Abstr. Am J Crit Care Med (Suppl) 159:A519

251. Geerts W, Cook D, Shelby R, Etchells E (2002) Venous thromboembolism and its prevention in critical care. J Crit Care 17:95-104

252. Attia J, Ray JG, Cook DJ, Douketis J, Ginsberg JS, Geerts WH (2001) Deep vein thrombosis and its prevention in critically ill adults. Arch Intern Med 161:1268-1279

253. King CS, Holley AB, Jackson JL, Shorr AF, Moores LK (2007) Twice vs three times daily heparin dosing for thromboembolism prophylaxis in the general medical population: A metaanalysis. Chest 131:507-516

254. Turpie AG, Hirsh J, Gent M, Julian D, Johnson J (1989) Prevention of deep vein thrombosis in potential neurosurgical patients: a randomized trial comparing graduated compression stockings alone or graduated compression stockings plus intermittent pneumatic compression with control. Arch Intern Med 149:679-681 
255. Vanek VW (1998) Meta-analysis of effectiveness of intermittent pneumatic compression devices with a comparison of thigh-high to knee-high sleeves. Am Surg 64:1050-1058

256. Agu O, Hamilton G, Baker D (1999) Graduated compression stocking in the prevention of venous thromboembolism. Br J Surg 86:992-1004

257. German Hip Arthroplasty Trial Group (GHAT) (1992) Prevention of deep vein thrombosis with low molecular-weight heparin in patients undergoing total hip replacement: a randomized trial. Arch Orthop Trauma Surg 111:110-120

258. Colwell CW Jr, Spiro TE, Trowbridge AA, Morris BA, Kwaan HC, Blaha JD, Comerota AJ, Skoutakis VA (1994) Use of enoxaparin, a lowmolecular-weight-heparin, and unfractionated heparin for the prevention of deep venous thrombosis after elective hip replacement: a clinical trial comparing efficacy and safety. J Bone Joint Surg Am 76:3-14

259. Geerts WH, Jay RM, Code KI, Chen E, Szalai JP, Saibil EA, Hamilton PA (1996) A comparison of low-dose heparin with low-molecular-weight heparin as prophylaxis against venous thromboembolism after major trauma. N Engl J Med 335:701-707

\section{Stress Ulcer Prophylaxis}

260. Basso N, Bagarani M, Materia A, Fiorani S, Lunardi P, Speranza V (1981) Cimetidine and antacid prophylaxis of acute upper gastrointestinal bleeding in high risk patients. Am J Surg 141:339-342

261. Bresalier RS, Grendell JH, Cello JP, Meyer AA (1987) Sucralfate versus titrated antacid for the prevention of acute stress-related gastrointestinal hemorrhage in critically ill patients. Am J Med 83:110-116

262. Poleski MH, Spanier AH (1986) Cimetidine versus antacids in the prevention of stress erosions in critically ill patients. Am J Gastroenterol 81:107-111

263. Stothert JC Jr, Simonowitz DA, Dellinger EP, Farley M, Edwards WA, Blair AD, Cutler R, Carrico CJ (1980) Randomized prospective evaluation of cimetidine and antacid control of gastric $\mathrm{pH}$ in the critically ill. Ann Surg 192:169-174

264. Cook DJ, Fuller HD, Guyatt GH, Marshall JC, Leasa D, Hall R, Winton TL, Rutledge F, Todd TJ, Roy P, et al. (1994) Risk factors for gastrointestinal bleeding in critically ill patients. N Engl J Med 330:377-381
265. Schuster DP, Rowley H, Feinstein S, McGue MK, Zuckerman GR (1984) Prospective evaluation of the risk of upper gastrointestinal bleeding after admission to a medical intensive care unit. Am J Med 76:623-629

266. Misra UK, Kalita J, Pandey S, Mandal SK, Srivastava M (2005) A randomized placebo controlled trial of ranitidine versus sucralfate in patients with spontaneous intracerebral hemorrhage for prevention of gastric hemorrhage. J Neurological Sciences 239:5-10

267. Friedman CJ, Oblinger MJ, Suratt PM, Bowers J, Goldberg SK, Sperling MH, Blitzer AH (1982) Prophylaxis of upper gastrointestinal hemorrhage in patients requiring mechanical ventilation. Crit Care Med 10:316-319

268. Hastings PR, Skillman JJ, Bushnell LS, Silen W (1978) Antacid titration in the prevention of acute gastrointestinal bleeding: A controlled, randomized trial in 100 critically ill patients. N Engl J Med 298:1041-1045

269. Cook DJ, Witt LG, Cook RJ, Guyatt GH (1991) Stress ulcer prophylaxis in the critically ill: A meta-analysis. Am J Med 91:519-257

270. Kahn JM, Doctor JN, Rubenfeld GD (2006) Stress ulcer prophylaxis in mechanically ventilated patients: integrating evidence and judgment using a decision analysis. Intensive Care Med 32:1151-1158

271. Cook D, Guyatt G, Marshall J, Leasa D, Fuller H, Hall R, Peters S, Rutledge F, Griffith L, McLellan A, Wood G, Kirby A (1998) A comparison of sucralfate and ranitidine for the prevention of upper gastrointestinal bleeding in patients requiring mechanical ventilation. N Engl J Med 338:791-797

272. Cook DJ, Reeve BK, Guyatt GH, Heyland DK, Griffith LE, Buckingham L, Tryba M (1996) Stress ulcer prophylaxis in critically ill patients: resolving discordant meta-analyses. JAMA 275:308-314

273. Levy MJ, Seelig CB, Robinson NJ, Ranney JE (1997) Comparison of omeprazole and ranitidine for stress ulcer prophylaxis. Dig Dis Sci 42:1255-1299

274. Quartin A, Hata JS, Frank WO, Bagin RG, Rock JA, Hepburn B, Laine L (2005) Randomized, doubleblind comparison of immediate-release omeprazole oral suspension versus intravenous cimetidine for the prevention of upper gastrointestinal bleeding in critically ill patients. Crit Care Med 33:760-765

\section{SDD References}

275. Vandenbroucke-Grauls CMJ, Vandenbroucke JP (1991) Effect of selective decontamination of the digestive tract on respiratory tract infections and mortality in the intensive care unit. Lancet 338:859-862

276. Selective Decontamination of the Digestive Tract Trialists' Collaborative Group (1993) Meta-analysis of randomised controlled trials of selective decontamination of the digestive tract. BMJ 307:525-532

277. Kollef M (1994) The role of selective digestive tract decontamination on mortality and respiratory tract infections. A meta-analysis. Chest 105:1101-1108

278. Heyland DK, Cook DJ, Jaeschke R, Griffith L, Lee HN, Guyatt GH (1994) Selective decontamination of the digestive tract: an overview. Chest 105:1221-1229

279. Hurley JC (1995) Prophylaxis with enteral antibiotics in ventilated patients: selective decontamination or selective cross-infection? Antimicrob Agents Chemother 39:941-947

280. D'Amico R, Pifferi S, Leonetti C, Torri V, Tinazzi A, Liberati A (1998) Effectiveness of antibiotic prophylaxis in critically ill adult patients: systematic review of randomised controlled trials. BMJ 316:1275-1285

281. Nathens AB, Marshall JC (1999) Selective decontamination of the digestive tract in surgical patients. A systematic review of the evidence. Arch Surg 134:170-176

282. Redman R, Ludington E, Crocker M, et al (2001) Analysis of respiratory and non-respiratory infections in published trials of selective digestive decontamination (abstract). Intensive Care Med 27(Suppl 1):S128

283. Safdar N, Said A, Lucey MR (2004) The role of selective digestive decontamination for reducing infection in patients undergoing liver transplantation: a systematic review and meta-analysis. Liver Transpl 10:817-827

284. Liberati A, D'Amico R, Pifferi S, Torri V, Brazzi L (2004) Antibiotic prophylaxis to reduce respiratory tract infections and mortality in adults receiving intensive care (Cochrane Review). In: The Cochrane Library, Issue 1. Chichester, UK: Wiley \& Sons, Ltd. 
285. Silvestri L, van Saene HKF, Milanese M, Gregori D (2005) Impact of selective decontamination of the digestive tract on fungal carriage and infection: systematic review of randomised controlled trials. Intensive Care Med 31:898-910

286. Silvestri L, Milanese M, Durì D, et al. (2005) Impact of SDD on bloodstream infections: a systematic review of randomized trials (abstract). Intensive Care Med 31(Suppl 1):S87

287. Hammond JMJ, Potgieter PD (1995) Is there a role for selective decontamination of the digestive tract in primarily infected patients in the ICU? Anaesth Intens Care 23:168-174

288. De Jonge E, Schultz M, Spanjaard L, Bossuvt P, Kesecioglu J (2003) Selective decontamination of digestive tract in intensive care. Lancet 362:2119-2120

289. De Jonge E, Schultz MJ, Spanjaard L, Bossuyt PM, Vroom MB, Dankert J, Kesecioglu J (2003) Effects of selective decontamination of the digestive tract on mortality and acquisition of resistance bacteria on intensive care: a randomised controlled trial. Lancet 362:1011-1016

290. Hammond JM, Potgieter PD, Saunders GL, Forder AA (1992) Double blind study of selective decontamination of the digestive tract in intensive care. Lancet 340:5-9

291. de la Cal MA, Cerda E, van Saene HKF, Garca-Hierro P, Negro E, Parra ML, Arias S, Ballesteros D (2004) Effectiveness and safety of enteral vancomycin to control endemicity of methicillin-resistant Staphylococcus aureus in a med$\mathrm{ical} /$ surgical intensive care unit. J Hospital Infect 56:175-183

292. Silvestri L, van Saene HKF, Milanese M, Fontana F, Gregori D, Oblach L, Piacente N, Blazic M (2004) Prevention of MRSA pneumonia by oral vancomycin decontamination: a randomised trial. Eur Respir J 23:921-926

293. Cerda E, Abella A, de la Cal MA, Lorente JA, Garca-Hierro P, van Saene HKF, Ala I, Aranguren A (2007) Enteral Vancomycin Controls Methicillin-resistant Staphylococcus Aureus Endemicity in an Intensive Care Burn Unit. A 9-Year Prospective Study. Ann Surg 245:397-407

\section{Consideration for Limitation of} Support

294. Curtis JR (2005) Interventions to improve care during withdrawal of life-sustaining treatments. J Palliative Medicine (Suppl 1) 8:S116-31

295. Thompson BT, Cox PN, Antonelli M, Carlet JM, Cassell J, Hill NS, Hinds CJ, Pimentel JM, Reinhart K, Thijs LG, American Thoracic Society, European Respiratory Society, European Society of Intensive Care Medicine, Society of Critical Care Medicine, Sociètède Rèanimation de Langue Française (2004) Challenges in end-of-life care in the ICU: statement of the 5th International Consensus Conference in Critical Care: Brussels, Belgium, April 2003: executive summary. Crit Care Med 32:1781-1784

296. Heyland DK, Tranmer J, O'Callaghan CJ, Gafni A (2003) The Seriously Ill Patient: Preferred Role in End of Life Decision Making. J Crit Care 18:3-10

297. Curtis JR, Engelberg RA, Wenrich MD, Shannon SE, Treece PD, Rubenfeld GD (2005) Missed opportunities during family conferences about end-of-life care in the intensive care unit. Am J Resp Crit Care Med 171:844-849

\section{Pediatric Considerations}

298. Watson RS, Carcillo JA, LindeZwirble WT, Clermont G, Lidicker J, Angus DC (2003) The epidemiology of severe sepsis in children in the United States. Am J Respir Crit Care Med 167:695-701

299. Goldstein B, Giroir B, Randolph A (2005) International pediatric sepsis consensus conference: Definitions for sepsis and organ dysfunction in pediatrics. Pediatr Crit Care Med 6:2-8

300. Pollard AJ, Britto J, Nadel S, DeMunter C, Habibi P, Levin M (1999) Emergency management of meningococcal disease. Arch Dis Child 80:290-296

301. den Brinker M, Joosten KF, Liem O, de Jong FH, Hop WC, Hazelzet JA, van Dijk M, Hokken-Koelega AC (2005) Adrenal insufficiency in meningococcal sepsis: Bioavailable cortisol levels and impact of interleukin- 6 levels and intubation with etomidate on adrenal function and mortality. Clin Endocrinol Metab 90:5110-5117
302. Kanter RK, Zimmerman JJ, Strauss RH, Stoeckel KA (1986) Pediatric emergency intravenous access. Evaluation of a protocol. Am J Dis Child 140:132-134

303. Ngo NT, Cao XT, Kneen R, Wills B, Nguyen VM, Nguyen TQ, Chu VT, Nguyen TT, Simpson JA, Solomon T, White NJ, Farrar J (2001) Acute management of dengue shock syndrome: a randomized double-blind comparison of 4 intravenous fluid regimens in the first hour. Clin Infect Dis 32:204-213

304. Carcillo JA, Davis AL, Zaritsky A (1991) Role of early fluid resuscitation in pediatric septic shock. JAMA 266:1242-1245

305. Han YY, Carcillo JA, Dragotta MA, Bills DM, Watson RS, Westerman ME, Orr RA (2003) Early reversal of pediatric-neonatal septic shock by community physicians is associated with improved outcome. Pediatrics 112:793-799

306. Ranjit S, Kissoon N, Jayakumar I (2005) Aggressive management of dengue shock syndrome may decrease mortality rate: a suggested protocol. Pediatr Crit Care Med 6:412-419

307. Wills BA, Nguyen MD, Ha TL, Dong TH, Tran TN, Le TT, Tran VD, Nguyen TH, Nguyen VC, Stepniewska K, White NJ, Farrar JJ (2005) Comparison of three fluid solutions for resuscitation in dengue shock syndrome. N Engl J Med 353:877-889

308. Dung NM, Day NP, Tam DT, Loan HT, Chau HT, Minh LN, Diet TV, Bethell DB, Kneen R, Hien TT, White NJ, Farrar JJ (1999) Fluid replacement in dengue shock syndrome: a randomized, double-blind comparison of four intravenous-fluid regimens. Clin Infect Dis 29:787-794

309. Ceneviva G, Paschall JA, Maffei F, Carcillo JA (1998) Hemodynamic support in fluid-refractory pediatric septic shock. Pediatrics 102:e19

310. Keeley SR, Bohn DJ (1988) The use of inotropic and afterloadreducing agents in neonates. Clin Perinatol 15:467-489

311. Barton P, Garcia J, Kouatli A, Kitchen L, Zorka A, Lindsay C, Lawless S, Giroir B (1996) Hemodynamic effects of i.v. milrinone lactate in pediatric patients with septic shock. A prospective, double-blinded, randomized, placebo-controlled, interventional study. Chest 109:1302-1312

312. Lindsay CA, Barton P, Lawless S, Kitchen L, Zorka A, Garcia J, Kouatli A, Giroir B (1998) Pharmacokinetics and pharmacodynamics of milrinone lactate in pediatric patients with septic shock. J Pediatr 132:329-334 
313. Irazuzta JE, Pretzlaff RK, Rowin ME (2001) Amrinone in pediatric refractory septic shock: An open-label pharmacodynamic study. Pediatr Crit Care Med 2:24-28

314. Powell KR, Sugarman LI, Eskenazi AE, Woodin KA, Kays MA, McCormick KL, Miller ME, Sladek CD (1991) Normalization of plasma arginine vasopressin concentrations when children with meningitis are given maintenance plus replacement fluid therapy. J Pediatr 117:515-522

315. Masutani S, Senzaki H, Ishido H, Taketazu M, Matsunaga T, Kobayashi T, Sasaki N, Asano H, Kyo S, Yokote Y (2005) Vasopressin in the treatment of vasodilatory shock in children. Pediatr Int 47:132-136

316. Booy R, Habibi P, Nadel S, de Munter C, Britto J, Morrison A, Levin M, Meningococcal Research Group (2001) Reduction in case fatality rate from meningococcal disease associated with improved healthcare delivery. Arch Dis Child85:386-390

317. Carcillo JA, Fields AI, American College of Critical Care Medicine Task Force Committee Members (2002) Clinical practice parameters for hemodynamic support of pediatric and neonatal patients in septic shock. Crit Care Med 30:1365-1378

318. Pizarro CF, Troster EJ, Damiani D, Carcillo JA (2005) Absolute and relative adrenal insufficiency in children with septic shock. Crit Care Med 33:855-859

319. Riordan FA, Thomson AP, Ratcliffe JM, Sills JA, Diver MJ, Hart CA (1999) Admission cortisol and adrenocorticotrophic hormone levels in children with meningococcal disease: evidence of adrenal insufficiency? Crit Care Med 27:2257-2261

320. De Kleijn ED, Joosten KF, Van Rijn B, Westerterp M, De Groot R, HokkenKoelega AC, Hazelzet JA (2002) Low serum cortisol in combination with high adrenocorticotrophic hormone concentrations are associated with poor outcome in children with severe meningococcal disease. Pediatr Infect Dis J 21:330-336

321. Markovitz BP, Goodman DM, Watson S, Bertoch D, Zimmerman J (2005) A retrospective cohort study of prognostic factors associated with outcome in pediatric severe sepsis: What is the role of steroids? Pediatr Crit Care Med 6:270-274
322. Hazelzet JA, de Kleijn ED, de Groot R (2001) Endothelial protein C activation in meningococcal sepsis. N Engl J Med 345:1776-1777

323. de Kleijn ED, de Groot R, Hack CE, Mulder PG, Engl W, Moritz B, Joosten KF, Hazelzet JA (2003) Activation of protein $\mathrm{C}$ following infusion of protein $\mathrm{C}$ concentrate in children with severe meningococcal sepsis and purpura fulminans: A randomized, double-blinded, placebocontrolled, dose-finding study. Crit Care Med 31:1839-1847

324. Nadel S, Goldstein B, Williams MD, Dalton H, Peters M, Macias WL, AbdAllah SA, Levy H, Angle R, Wang D, Sundin DP, Giroir B, Researching severe Sepsis and Organ dysfunction in children: a gLobal perspective (RESOLVE) study group (2007) Drotrecogin alfa (activated) in children with severe sepsis: A multicentre phase III randomized controlled trial. Lancet 369:836-843

325. Krafte-Jacobs B, Sivit CJ, Mejia R, Pollack MM (1995) Catheterrelated thrombosis in critically ill children: comparison of catheters with and without heparin bonding. J Pediatr 126:50-54

326. Pierce CM, Wade A, Mok Q (2000) Heparin-bonded central venous lines reduce thrombotic and infective complications in critically ill children. Intensive Care Med 26:967-972

327. Chaïbou M, Tucci M, Dugas MA, Farrell CA, Proulx F, Lacroix J (1998) Clinically significant upper gastrointestinal bleeding acquired in a pediatric intensive care unit: A prospective study. Pediatrics 102:933-938

328. Gauvin F, Dugas MA, Chaïbou M Morneau S, Lebel D, Lacroix J (2001) The impact of clinically significant upper gastrointestinal bleeding in a pediatric intensive care unit. Pediatr Crit Care Med 2:294-298

329. Foland JA, Fortenberry JD, Warshaw BL, Pettignano R, Merritt RK, Heard ML, Rogers K, Reid C, Tanner AJ, Easley KA (2004) Fluid overload before continuous hemofiltration and survival in critically ill children: A retrospective analysis. Crit Care Med 32:1771-1776
330. Branco RG, Garcia PC, Piva JP, Casartelli CH, Seibel V, Tasker RC (2005) Glucose level and risk of mortality in pediatric septic shock. Pediatr Crit Care Med 6:470-472

331. Faustino EV, Apkon M (2005) Persistent hyperglycemia in critically ill children. J Pediatr 146:30-34

332. Cam PC, Cardone D (2007) Propofol infusion syndrome. Anaesthesia 62:690-701

333. Parke TJ, Stevens JE, Rice AS, Greenaway CL, Bray RJ, Smith PJ, Waldmann CS, Verghese C (1992) Metabolic acidosis and fatal myocardial failure after propofol infusion in children: five case reports. BMJ 305:613-616

334. Lacroix J, Hébert PC, Hutchison JS, Hume HA, Tucci M, Ducruet T, Gauvin F, Collet JP, Toledano BJ, Robillard P, Joffe A, Biarent D, Meert K, Peters MJ, TRIPICU Investigators, Canadian Critical Care Trials Group, Pediatric Acute Lung Injury and Sepsis Investigators Network (2007) Transfusion strategies for patients in pediatric intensive care units. New Engl J Med 256:1609-1619

335. El-Nawawy A, El-Kinany H, Hamdy El-Sayed M, Boshra N (2005) Intravenous polyclonal immunoglobulin administration to sepsis syndrome patients: a prospective study in a pediatric intensive care unit. J Trop Pediatr 51:271-278

336. Meyer DM, Jessen ME (1997) Results of extracorporeal membrane oxygenation in children with sepsis. The Extracorporeal Life Support Organization. Ann Thorac Surg 63:756-761

337. Goldman AP, Kerr SJ, Butt W, Marsh MJ, Murdoch IA, Paul T, Firmin RK, Tasker RC, Macrae DJ (1997) Extracorporeal support for intractable cardiorespiratory failure due to meningococcal disease. Lancet 349:466-469

\section{Summary and Future Directions}

338. Cinel I, Dellinger RP (2006) Guidelines for severe infections: are they useful. Curr Opin Crit Care 12:483-488 
339. Levy MM, Pronovost PJ, Dellinger RP, Townsend S, Resar RK, Clemmer TP, Ramsay G (2004) Sepsis change bundles: converting guidelines into meaningful change in behavior and clinical outcome. Crit Care Med [Suppl] 32:S595-S597

\section{Appendicies}

340. Meduri GU, Golden E, Freire AX, Taylor E, Zaman M, Carson SJ, Gibson M, Umberger R (2007)

Methylprednisolone infusion in early severe ARDS: Results of a randomized controlled trial. Chest 131:1954-1963
341. Steinberg KP, Hudson LD, Goodman RB, Hough CL, Lanken PN, Hyzy R, Thompson BT, Ancukiewicz M, National Heart, Lung and Blood Institute Acute Respiratory Distress Syndrome (ARDS) Clinical Trials Network (2006) Efficacy and safety of corticosteroids for persistent acute respiratory distress syndrome. N Engl J Med 354:1671-1684 